\title{
WestVirginiaUniversity
}

THE RESEARCH REPOSITORY @ WVU

Graduate Theses, Dissertations, and Problem Reports

2006

\section{Cooperative diversity using MIMO systems}

Ramachandran Rajagopalan
West Virginia University

Follow this and additional works at: https://researchrepository.wvu.edu/etd

\section{Recommended Citation}

Rajagopalan, Ramachandran, "Cooperative diversity using MIMO systems" (2006). Graduate Theses, Dissertations, and Problem Reports. 1785.

https://researchrepository.wvu.edu/etd/1785

This Thesis is protected by copyright and/or related rights. It has been brought to you by the The Research Repository @ WVU with permission from the rights-holder(s). You are free to use this Thesis in any way that is permitted by the copyright and related rights legislation that applies to your use. For other uses you must obtain permission from the rights-holder(s) directly, unless additional rights are indicated by a Creative Commons license in the record and/ or on the work itself. This Thesis has been accepted for inclusion in WVU Graduate Theses, Dissertations, and Problem Reports collection by an authorized administrator of The Research Repository @ WVU. For more information, please contact researchrepository@mail.wvu.edu. 


\title{
Cooperative Diversity Using MIMO Systems
}

\author{
by \\ Ramachandran Rajagopalan \\ Thesis submitted to the \\ College of Engineering and Mineral Resources \\ at West Virginia University \\ in partial fulfillment of the requirements \\ for the degree of \\ Master of Science \\ in \\ Electrical Engineering
}

Daryl .S. Reynolds, Ph.D., Chair

Brian .D. Woerner, Ph.D., Co-Chair

Matthew .C. Valenti, Ph.D

Lane Department of Computer Science and Electrical Engineering

Morgantown, West Virginia

2006

Keywords: Cooperative Diversity, MIMO, Decode-and-Forward, Space-Time Codes, Information Outage Probability

Copyright 2006 Ramachandran Rajagopalan 


\author{
Abstract \\ Cooperative Diversity using MIMO systems \\ by \\ Ramachandran Rajagopalan \\ Master of Science in Electrical Engineering \\ West Virginia University \\ Brian .D. Woerner, Ph.D., Co-Chair \\ Daryl .S. Reynolds, Ph.D., Chair
}

Multipath fading is one of the primary factors for degrading the performance in a wireless network. Information theoretic and past research suggest the use various diversity techniques to combat fading in wireless networks. Antenna diversity, a form of diversity technique, when incorporated in a wireless transceiver increases the system capacity and is one of the effective methods to combat fading in wireless systems. Also, recent research by Laneman et.al., Sendonaris et.al. suggests that cooperation among users in a wireless networks is an effective approach for a better signal reception in multipath fading environments. The diversity gains obtained by cooperation among the users of a wireless network is termed as cooperative diversity. Although, prior research in cooperative diversity considers users equipped with single antenna, in practical scenarios users may be able to accommodate multiple antennas due to the recent advanced research in semiconductor industry. Hence, the primary purpose of this thesis is to design, simulate and analyze an end-end performance of multi-antenna wireless systems employing cooperative multi antenna relay nodes so as to exploit the cooperative diversity and antenna diversity simultaneously in a wireless networks. Three main contributions to the area of cooperative multiple-input multiple-output (MIMO) wireless systems is presented in this thesis. First, we perform information theoretic analysis to study the impact of antenna arrays on cooperative wireless networks and propose the best possible distribution of antenna arrays among the three terminals of a simple three terminal cooperative relay network. Second, we design, simulate, and analyze a cooperative multiple-input multipleoutput (MIMO) wireless systems employing orthogonal space-time block codes as proposed by Alamouti in 1998 with a decode-and-forward (DF) relay terminal. We implement a maximal ratio combining receiver that provides almost twice the diversity gain with respect to point-point multiple input multiple output link. Finally, we implement a practical receiver for cooperative reception using multiple antennas at all nodes based on Bell-Labs Layered Space Time architecture (BLAST). We incorporate a practical adaptive decode-and-forward (DF) relaying technique for reliable signal retransmission for both Alamouti space-time coding and the BLAST schemes. Results presented in terms of bit error rates and throughput show that remarkable performance gains are achievable by combining the concepts drawn from space-time coding, cooperative relaying and array processing. 
To My Family 


\section{Acknowledgments}

I am indebted to my advisor and mentor Dr. Woerner for inspiring me and giving me the freedom to work in the area of wireless communications. The idea behind this thesis had formulated from his course on Multi-Antenna Systems. His consistent encouragement, support and advice have helped me to develop my research capabilities and writing skills. His attitude towards colleagues, staff and students will serve as a role model throughout my career.

I would like to thank Dr. Reynolds and Dr. Valenti for their innovative suggestions. I wish to acknowledge the patience shown by both of them towards my frequency of knocking the door to clarify my questions. In short I learnt the art of working smartly and efficiently from them.

I would like to thank my lab mate and teacher at WCRL Mrs.Kanchan Panse for her suggestions, technical discussions, motivation during my studies and all WCRL'ers for their technical insights, my roommates and friends whom I have known from my first day in Morgantown for their support and making my stay more comfortable here.

I have no words to express my feelings for the support my family extended during this studies and my parents in particular for offering me a good education. I would like to thank my sister, attai's, chittapa's, chitti's, mama and mami's who have been constantly motivating me to pursue higher studies. I would also like to thank all my little cousins for their friendly support since my childhood. Finally, I would dedicate this thesis to my grandmother who has always motivated and supported me from the very beginning of my education. 


\section{Contents}

Acknowledgments

List of Figures

Notation $\quad$ ix

1 Introduction 1

1.1 Thesis Outline . . . . . . . . . . . . . . . . . . . . . . . . . . . . 3

1.2 Contributions ............................ . 4

2 Multiple-Input Multiple-Output Wireless Communication System Overview 6

2.1 Fading Channels . . . . . . . . . . . . . . . . . 7

2.1.1 Fading Effects due to Delay spread . . . . . . . . . . . . 8

2.1.2 Fading Effects due to Doppler spread . . . . . . . . . . . . 8

2.2 MIMO Flat Fading Channel . . . . . . . . . . . . . . . . . . . 9

2.3 Capacity and Information Outage Probability for a Few Special Cases . . . . 11

2.3.1 Single-Input Single-Output Channel (SISO) . . . . . . . . . . . . . . 11

2.3.2 Single-Input Multiple-Output Channel (SIMO) . . . . . . . . . . . . 11

2.3.3 Multiple-Input Single-Output Channel (MISO) . . . . . . . . . . . . 12

2.4 Simulation Results . . . . . . . . . . . . . . . . . . . . 13

2.5 Performance of Space-Time Block Codes . . . . . . . . . . . . . . . . . . 15

2.5.1 Space-Time Block Codes Using MISO System . . . . . . . . . . . . . 16

2.5.2 Space-Time Block Codes Using MIMO Systems . . . . . . . . . . . . 18

2.5.3 Simulation Results . . . . . . . . . . . . . . . . . . . 20

2.6 Performance Analysis of BLAST . . . . . . . . . . . . . . . . . . . . . . . . . . . . . . . . . . . . . . .

2.7 Simulation Results . . . . . . . . . . . . . . . . . . . . 24

2.8 Chapter Summary . . . . . . . . . . . . . . . . . . . . 26

3 Impact of Multiple Antennas on Cooperative Relay Networks $\quad 27$

3.1 Relay Networks . . . . . . . . . . . . . . . . . . . . . . . 28

3.2 Relay Network Outage Analysis: Code Combining . . . . . . . . . . . . . . . 29

3.2.1 Array at the Source . . . . . . . . . . . . . . . . . 30

3.2 .2 Array at the Relay . . . . . . . . . . . . . . . . . . . . . . . . . . 32

3.2 .3 Array at the Destination . . . . . . . . . . . . . . . 33 
3.2 .4 Numerical Results . . . . . . . . . . . . . . . . . . . . . . 34

3.3 Relay Network Outage Analysis: Diversity Combining . . . . . . . . . . . . . 38

3.3.1 Array at the Destination . . . . . . . . . . . . . . . . 39

3.3.2 Array at the Relay . . . . . . . . . . . . . . . . . . . 41

3.3.3 Array at the Source . . . . . . . . . . . . . . . . . . . . 42

3.3 .4 Numerical Results . . . . . . . . . . . . . . . . . . . . . 43

3.4 Relay Network Outage Analysis: Arrays at multiple nodes . . . . . . . . . . 44

3.4.1 Code Combining . . . . . . . . . . . . . . . . . 44

3.4.2 Diversity Combining . . . . . . . . . . . . . . . 46

3.5 Code Combining Vs Diversity Combining . . . . . . . . . . . . . . . . . . . 48

3.6 Cooperation Rate . . . . . . . . . . . . . . . . . . . . . . . 49

3.7 Chapter Summary . . . . . . . . . . . . . . . . . . . . 52

4 Practical Cooperative Space-Time Architectures $\quad 53$

4.1 Cooperative Space-Time Transmission Architectures . . . . . . . . . . . . . . 54

4.1.1 Cooperative Transmission Using STBC . . . . . . . . . . . . . . . 54

4.1.2 Cooperative VBLAST Transmission . . . . . . . . . . . . . . 55

4.2 Simulation Results . . . . . . . . . . . . . . . . . . . . 56

4.3 Chapter Summary . . . . . . . . . . . . . . . . . . 6 61

5 Conclusions $\quad \mathbf{6 2}$

5.1 Summary of Results and Conclusion _ . . . . . . . . . . . . . . . . 62

5.2 Future Work . . . . . . . . . . . . . . . . . . . . . 63

$\begin{array}{ll}\text { References } & 65\end{array}$ 


\section{List of Figures}

2.1 Block diagram of a generalized MIMO system . . . . . . . . . . . . . . . 9

$2.210 \%$ Outage capacity of MIMO systems with varying antenna configurations in slowly varying rayleigh fading environment. . . . . . . . . . . . . 13

2.3 Outage Probability of MIMO systems with varying antenna configurations in slowly varying rayleigh fading environment with $\mathrm{R}=1 \mathrm{bit} /$ channel-use. . . . .

2.4 Comparison of Outage Probability of MIMO systems obtained via Monte Carlo (MC) integration method and numeric integration (NC) method for different antenna configurations in slowly varying rayleigh fading environment with $\mathrm{R}=1$ bit/channel-use. . . . . . . . . . . . . . . . . . . . . 14

2.5 Block diagram of full rate orthogonal space time block coding scheme. . . . . 16

2.6 Bit error rate of orthogonal space time block code with $\mathrm{R}=2$ bits/channel-use. 20

2.7 Normalized Throughput of orthogonal space time block code. . . . . . . . . . 21

2.8 Block diagram of V-BLAST architecture. . . . . . . . . . . . . . . . 22

2.9 Bit error rate of VBLAST for $M_{T}=2, M_{R}=2$ in rayleigh fading with $\mathrm{R}=4$ bits/channel-use. . . . . . . . . . . . . . . 25

2.10 Normalized throughput of VBLAST for $M_{T}=2, M_{R}=2$ in rayleigh fading. . $\quad 26$

3.1 A three terminal relay network. . . . . . . . . . . . . . . . . . . 28

3.2 Outage probability performance comparison between Monte Carlo integration (symbols) and numerical integration (dashed lines) for the configurations $n_{S}=$ $1, n_{R}=1, n_{D}=2, n_{S}=1, n_{R}=2, n_{D}=1$ and $n_{S}=2, n_{R}=1, n_{D}=1$ with code combining in rayleigh fading environment $(\alpha=0.5)$ with $\mathrm{R}=1$ bit/channel-use. . . . . . . . . . . . . . . . . .

3.3 Outage probability performance (Monte Carlo integration) with $n_{S}=1 ; n_{R}=$ 1 and $n_{D}=2,3,4,5,6$ antennas in rayleigh fading environments $(\alpha=0.5)$ with $\mathrm{R}=1$ bit/channel-use. . . . . . . . . . . . . . . . . . . .

3.4 Outage probability performance (numerical integration) with $n_{S}=1 ; n_{R}=$ $2,3,4,5,6$ and $n_{D}=1$ antennas for code combining scheme in rayleigh fading environment $(\alpha=0.5)$ with $\mathrm{R}=1$ bit/channel-use. . . . . . . . . . .

3.5 Outage probability performance with $n_{S}=2,3,4,5,6 ; n_{R}=1$ and $n_{D}=1$ antennas for code combining scheme in rayleigh fading environment $(\alpha=0.5)$.

3.6 Outage probability performance by varying the number of antennas at each node from 2 to 6 in rayleigh fading environment $(\alpha=0.5)$ with $\mathrm{R}=1 \mathrm{bit} /$ channeluse. . . . . . . . . . . . . . . . . . . 38 
3.7 Outage probability performance of diversity combining (numerical integration) by varying the number of antennas at each node from 2 to 6 with $R=1$ bit/channel-use. . . . . . . . . . . . . . . . .

3.8 Outage probability performance of code combining scheme obtained via Monte Carlo simulation by incorporating antenna arrays at multiple nodes simultaneously with $\mathrm{R}=1 \mathrm{bit} /$ channel-use. $(\alpha=0.6) \ldots \ldots \ldots$. . . . . .

3.9 Outage probability performance of diversity combining scheme obtained via Monte Carlo integration by incorporating multiple antennas in rayleigh fading environment with $\mathrm{R}=1 \mathrm{bit} /$ channel-use. . . . . . . . . . . . . . .

3.10 Comparison of code combining and diversity combining scheme using Monte Carlo integration with $\mathrm{R}=1$ bit/channel-use. . . . . . . . . . . . . . . .

3.11 Outage probability performance of code combining scheme obtained by numerical integration with $\left(n_{S}=1, n_{R}=2, n_{D}=1\right)$ at various $\mathrm{SNR}$ with $\mathrm{R}=1$ bit/channel-use. . . . . . . . . . . . . . . . . .

3.12 Outage probability performance of code combining scheme obtained by Monte Carlo integration (solid lines) and numerical integration (dashed lines) for different antennas $(L=2,3,4)$ at a fixed $\mathrm{SNR}$ of $5 \mathrm{~dB}$ with $\mathrm{R}=1 \mathrm{bit} /$ channeluse. . . . . . . . . . . . . . . . . . . . .

3.13 Outage probability performance of code combining scheme obtained via Monte Carlo integration by incorporating multiple antennas in rayleigh fading environment with $\mathrm{R}=1 \mathrm{bit} /$ channel-use. . . . . . . . . . . . . . . . .

4.1 BER and diversity performance of cooperative DF STBC. The BER curve of $2: 2 \mathrm{STBC}$ is plotted for comparison, as is the performance of maximum ratio combining with 8 antennas with $\mathrm{R}=1 \mathrm{bit} /$ channel-use . . . . . . . . .

4.2 Throughput performance of cooperative DF STBC. The Throughput of 2:2 STBC is plotted for comparison . . . . . . . . . . . . . . .

4.3 BER performance of cooperative DF VBLAST using ZF with and without ordered SIC with $\mathrm{R}=2$ bits/channel-use. The BER curve of 2:2 VBLAST for the same detection scheme is plotted for comparison . . . . . . . . . . .

4.4 BER performance of cooperative DF VBLAST using MMSE with and without ordered SIC with $\mathrm{R}=2$ bits/channel-use. The BER curve of 2:2 VBLAST for the same detection scheme is plotted for comparison. . . . . . . . . . .

4.5 Throughput performance of cooperative DF VBLAST using ZF, with and without ordered SIC. The BER curve of 2:2 VBLAST for the same detection scheme is plotted for comparison . . . . . . . . . . . . . . .

4.6 Throughput performance of cooperative DF VBLAST using MMSE, with and without ordered SIC. The BER curve of 2:2 VBLAST for the same detection scheme is plotted for comparison . . . . . . . . . . . . . .

4.7 Throughput comparison of cooperative STBC and ZF,MMSE VBLAST with and without ordered SIC. 


\section{Notation}

We use the following notation and symbols throughout this thesis.

$(\cdot)^{H} \quad$ : Complex conjugate transpose

$(\cdot)^{*} \quad:$ Complex conjugate

$\mathcal{N}_{c}\left(0, \sigma^{2}\right)$ : Complex Gaussian distribution with zero mean and variance $\sigma^{2}$

$\mathrm{E}\{\cdot\} \quad$ : Expectation operator

$\operatorname{Tr}\{\mathbf{A}\} \quad$ : Trace of $\mathbf{A}$

$p(x \mid y) \quad$ : Probability of $x$ conditioned on $y$

$\|\cdot\| \quad$ : Euclidian norm

$\boldsymbol{I}_{K} \quad: K \times K$ Identity matrix

$\operatorname{det}(\cdot) \quad:$ Determinant operator

$\Re\{\cdot\} \quad$ : Real part of the argument

$\Im\{\cdot\} \quad$ : Imaginary part of the argument

$|\cdot|$ : Cardinality of a set

Bold uppercase letters denote matrices while bold lower case letters denote vectors. 


\section{Chapter 1}

\section{Introduction}

Multipath fading is one of the most challenging phenomena in wireless communications. The common solution to combat multipath fading is through the use of diversity techniques, which are classified as follows.

- Time Diversity: Transmitting the same information at time spacing that exceeds the coherence time (defined as the time duration over which two received signals have a strong potential for amplitude correlation) of the channel, so that multiple repetitions of the signal will be undergo independent fading conditions, provide the time diversity effect. Error correction codes with interleaving is one of the common forms to realize the time diversity in wireless channel. But, the use of error correction codes with large interleavers to achieve the time diversity effect results in bandwidth expansion and large delays.

- Frequency Diversity: Frequency diversity is achieved by transmitting the same signal over different carrier frequencies whose separation is larger than the coherence bandwidth (the range of frequencies in which the channel exhibits a flat response) of the channel. This form of diversity also suffers from bandwidth expansion.

- Spatial Diversity: Spatial diversity utilizes multiple antennas at the transmitter or receiver or at both the ends of point-to-point links to achieve diversity. With sufficient spacing, the signals transmitted from multiple antennas experience independent fading and can be coherently combined at the receiver using appropriate signal processing 
techniques to achieve the spatial diversity. This form of diversity does not require extra spectrum occupancy.

In $[1,2]$, the impact of the above mentioned diversity schemes are studied extensively in a point-point communications scenario. Because diversity is generally applied in a nonergodic setting, where the Shannon capacity does not exist, the benefits of diversity are explained using the concepts of information outage probability or outage capacity. Recent research $[3,4,5,6]$ indicates a special form of spatial diversity called cooperative diversity as an effective approach for mitigating multipath fading in wireless networks.

Cooperative diversity is achieved in a relay network, wherein a relay assists the source in the transmission of a message to the destination. Often, the relay operates in a time-division duplex (TDD) mode i.e. we allocate separate time slots to distinguish between the source and relay transmissions, which greatly simplifies system implementation. In addition, the source and relay usually transmit orthogonal signals. Orthogonality could be achieved by using different time slots, orthogonal spreading codes, or an orthogonal space-time code. While it is possible to engineer systems assuming non-orthogonal source and relay transmissions, which leads to multiple access interference (MAI) in the system and requires complex receiver designs to mitigate the MAI. Hence, for ease of exposition we assume that this orthogonality condition is met for the remainder of this discussion.

Two fundamental methods of orthogonal relaying considered in the literature are amplifyand-forward (AF) and decode-and-forward (DF) relaying [5]. In AF relaying, the relay simply retransmits an amplified version of the received signal, without making hard decisions on the message. The main drawback of the AF relaying protocol is that the noise on the source-relay channel is present in the retransmitted signal. In conventional (nonadaptive) DF relaying, the relay decodes and retransmits a reconstructed version of the received signal. The main drawback in this relaying scheme is that the relay could retransmit an incorrectly decoded message, though this problem can be alleviated through the use of an error detecting code. Coded cooperation is an efficient variation on the idea of DF relaying [7]. Whereas a standard DF protocol might have the source and relay transmit the same codeword (through repetition coding), a coded cooperation protocol would have the source and relay transmit different 
parts of the same codeword (through incremental redundancy).

Prior work on cooperative diversity has generally considered networks with only a single antenna at each node, while the use of antenna arrays at the individual nodes has gone largely unnoticed. In [8], the source and relay nodes form a distributed antenna array and are used to send a space-time codeword. However, each node contains only a single antenna. In [9], the base station (source) uses two antennas to send the 2-by-2 Alamouti space-time code to a pair of handsets (relay and destination). Each handset is equipped with one antenna, but is able to mimic a two antenna receiver by exchanging information. While this is an example of a relay network with an array, only one configuration was considered and a more generalized study should include the possibility of placing the array at a different terminal or using arrays of more than just two antennas. In [10], a unifying analysis is presented for the case of AF protocols with multiple antenna terminals, but DF was not considered. In [11], DF protocols with multiple antenna terminals was considered, but explicit, closed form expressions for outage probability were not given (instead numerical results relied on Monte Carlo integration). In [7], code combining techniques for networks with single antenna terminals is considered in depth. [12] provides a unified analysis of AF and DF protocols and proposes new adaptive relaying protocols using code and diversity combining techniques. While the adaptive cooperative diversity for the multiple relay case is considered to be a generalization of hybrid-ARQ, again only terminals with a single antenna are considered.

Hence, in this thesis we study the joint impact of cooperative diversity and antenna diversity in a simple three terminal relay network. We investigate the best possible distribution of antenna arrays from an information theoretic perspective in a three terminal cooperative relay network. This is followed by simulation of cooperative relay network with multi-antenna terminals employing practical space time codes.

\subsection{Thesis Outline}

The thesis is organized as follows. Chapter 2 gives an overview of the point-to-point multiple-input multiple-output (MIMO) wireless communication systems. It begins with the description of quasi-static frequency flat MIMO wireless channel modeled for a typical 
wireless local area network (WLAN) setting. This is followed by the review of basic information theoretic results for the point-to-point MIMO channel in terms of ergodic capacity and information outage probability. In the end we discuss the ubiquitous STBC and VBLAST architectures.

Chapter 3 describes the the description of the system model with multiple antennas in a simple three terminal adaptive decode-and- forward (DF) relay network. After the description of the system model, Chapter 3 builds upon the information theoretic results obtained in Chapter 2, to investigate the impact of an antenna array on a relay network for two different combining schemes namely diversity combining and code combining at the destination. Further we incorporate multiple antennas at more than one node in a three terminal relay network and investigate the performance in terms of information outage probability for the code combining scheme. Some special cases of diversity combining scheme are also investigated.

Chapter 4 gives a brief description of novel cooperative space time architectures with simple adaptive DF relaying incorporating orthogonal space time block code and VBLAST transmission schemes. Results presented in terms of BER and throughput highlight the gains involved due to cooperative transmission.

Chapter 5 summarizes the thesis and presents a brief discussion on possible directions for future research.

\subsection{Contributions}

The contributions of this thesis are to

- Analyze the performance of multiple antennas in a three terminal wireless network from an information-theoretic perspective using simple adaptive decode-and-forward (DF) at the relay for two different combining schemes at the destination.

- Propose the best possible distribution of multiple antennas among the three terminals of a wireless network employing diversity combining and code combining scheme.

- Analyze the performance of employing practical code structures with repetition based 
cooperative diversity using MIMO systems form the concepts of STBC proposed by Alamouti [13] and Foschini's VBLAST architecture [1]. 


\section{Chapter 2}

\section{Multiple-Input Multiple-Output Wireless Communication System Overview}

Information theory is the framework to study the performance limits in wireless communication system. Capacity of a channel (Shannon capacity), defined as the maximum rate at which low error probability is achieved, forms the basic performance measure. For fading wireless channels several notions of channel capacity are developed to study the performance limits of wireless channels. In a fast fading environment, the channel coherence time is less than the symbol period. The Shannon capacity exists for the fast fading channels as the transmitted codeword experiences all the fading states and this capacity is called as ergodic capacity. In a typical WLAN or a cellular network we assume a quasi-static (block fading) frequency non-selective fading, i.e. the coherence time of channel is greater than the symbol duration, which means that the fading is constant for the entire block that is transmitted. Thus, no codeword in a block fading channel can experience all the fading states of the channel and thereby the notion of ergodicity is absent [2, 14]. Hence, outage capacity or information outage probability forms the basic performance measure of wireless networks operating in a slow fading environment.

In this chapter we present an information theoretic overview of multiple-input multipleoutput (MIMO) wireless systems that demonstrates an enormous capacity increase over the 
conventional single antenna wireless system $[1,15,16]$. We consider a quasi-static flat fading channel where the channel state information is known only to the receiver. In particular, for a given transmitted signal power, we explore the outage capacity and information outage probability for various antenna array architectures and compare the MIMO system with receive diversity, transmit diversity and single antenna systems. These results will form a motivation factor in the design of practical multiple-antenna wireless system design. Following the discussion of information theory of MIMO systems we present the simulation results of practical wireless system using Orthogonal Space-Time Block Codes (OSTBC) and Bell LAbs Space Time (BLAST) architecture. These results are evaluated in terms of bit error rate (BER) and throughput.

\subsection{Fading Channels}

The term fading is one of the fundamental limitations in the performance of wireless systems. The multipath fading arises at a specific location due to the interaction of multiple reflections of electromagnetic waves from various objects.

Different propagation models like the large scale propagation model and fading or small scale model have been specified in the literature [17]. The large scale model focuses on predicting the received signal strength as function of distance from the transmitter. The small scale or the fading model is used for characterizing the rapid fluctuations of amplitudes, phases or multipath delays of the received signal over very short travel distances (a few wavelengths) or short time durations (on the order of seconds), so that the large scale path loss effects may be ignored. As the nodes of a mobile wireless local area network (WLAN) move over very short distances, the received signal fluctuates rapidly giving rise to small-scale fading. The three most important effects caused by small scale fading are

- Rapid changes in signal strength over small distances or time interval.

- Random frequency variations due to varying Doppler shifts on different multipath signals.

- Time dispersion (echoes) caused by multipath propagation delays. 
A transmitted signal may experience different types of fading depending upon the relation between the channel parameters (delay spread and Doppler spread) and the signal parameters (bandwidth and signal period). Hence we further classify the type of fading that a signal can experience based on two channel parameters called the delay spread and Doppler spread, which are independent propagation mechanisms. The delay spread leads to time dispersion and frequency selective fading. The Doppler spread accounts for the frequency dispersive and time selective nature of the channel.

\subsubsection{Fading Effects due to Delay spread}

The time dispersion due to multipath, decides whether the transmitted signal undergoes frequency non-selective (flat) fading or frequency selective fading.

- Flat Fading: A signal undergoes flat fading if the bandwidth of the signal is less than the coherence bandwidth and if the delay spread is less than the symbol duration.

- Frequency-selective Fading: A signal undergoes frequency selective fading if the coherence bandwidth is less than the bandwidth of the signal and if the symbol period is less than the delay spread. There is channel induced intersymbol interference (ISI).

\subsubsection{Fading Effects due to Doppler spread}

Comparisons of the base band signal variations with respect to the channel variations leads to the classification of the wireless channel as fast fading or slow fading channels.

- Fast Fading: A signal undergoes fast fading if the coherence time of the channel is less than the symbol duration. The Doppler spread is high and the channel variations are faster than the baseband signal variations.

- Slow Fading: A signal undergoes slow fading if the coherence time is larger than the duration of the symbol. The Doppler spread is low and the channel variations are slower than the base band signal variations.

In the rest of our discussions we consider only slowly varying flat fading channels. 


\section{$2.2 \quad$ MIMO Flat Fading Channel}

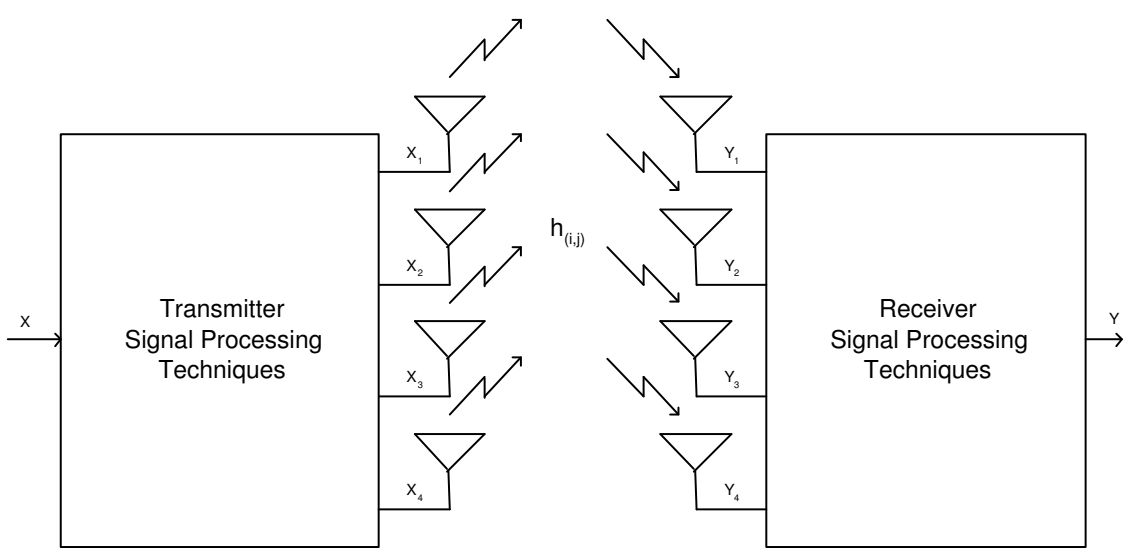

Figure 2.1: Block diagram of a generalized MIMO system

A point-point multiple-input multiple-output (MIMO) communication system with $M_{T}$ transmit antennas and $M_{R}$ receive antennas is shown in Fig. 2.1. The discrete-time matrix vector of the received signal for the given system is of the form

$$
\mathbf{y}=\sqrt{\frac{E_{s}}{M_{T}}} \mathbf{H} \mathbf{s}+\mathbf{n}
$$

where

$$
\mathbf{y}=\left[\begin{array}{lllll}
y_{1} & y_{2} & \cdot & \cdot & y_{M_{R}}
\end{array}\right]^{T}
$$

is a vector of matched filter outputs, one for each receive antenna ( $T$ is the transpose of the given vector) and $E_{s}$ is the total average energy available at the transmitter over a symbol period. We assume that the covariance matrix of $\mathbf{s}, \mathrm{E}\left\{\mathbf{s s}^{H}\right\}=\mathbf{I}$ in order to allocate equal power across all the transmit antennas over a symbol period.

The flat fading MIMO channel is modeled by the channel matrix $\mathbf{H}$ and is given by

$$
\mathbf{H}=\left[\begin{array}{ccccc}
h_{1,1} & h_{1,2} & \cdot & \cdot & h_{1, M_{T}} \\
h_{2,1} & h_{2,2} & \cdot & \cdot & h_{2, M_{T}} \\
\cdot & \cdot & \cdot & \cdot & \cdot \\
\cdot & \cdot & \cdot & \cdot & \cdot \\
h_{M_{R}, 1} & h_{M_{R}, 2} & \cdot & \cdot & h_{M_{R}, M_{T}}
\end{array}\right]
$$


in which the $(i, j)$ th entry represents the complex fade coefficient from the transmit antenna $j, j=\left\{1,2, \ldots, M_{T}\right\}$ to receive antenna $i, i=\left\{1,2, \ldots, M_{R}\right\}$, where $M_{T}$ and $M_{R}$ represent the number of antennas at the transmitter and receiver respectively. For further analysis we assume that the channel fade coefficients $h_{i, j}$ are independent identically distributed (i.i.d) zero mean complex Gaussian with a variance of 0.5 per dimension. We also assume that the noise components that affecting different receivers are independent each with variance $\frac{N_{0}}{2}$ per dimension.

The channel is assumed to be slow, flat and the fade coefficients associated with the multiple antennas are assumed to be uncorrelated. We assume a rich scattering environment such that the fade coefficients are Rayleigh distributed. These ideal channel conditions represent may represent the indoor channels between fixed terminal which form a part of the WLAN. The channel $\mathbf{H}$ is assumed to be quasi-static (i.e. $\mathbf{H}$ is a random matrix and the fading coefficients of the channel matrix $\mathbf{H}$ are chosen such that they are constant over entire block length and change independently between the consecutive blocks) such that the time coherence of the channel is larger than the symbol duration. Equal power is assumed to be allocated to all the transmit antennas.

In [1] Foschini and Gans study the performance of multi-antenna wireless systems in rayleigh fading environments in terms of channel capacity. A convenient formula for describing the capacity of point-point MIMO wireless system in a Rayleigh fading environment is given by

$$
C=\log \left(\operatorname{det}\left(I_{M_{R}}+\frac{\rho}{M_{T}} \mathbf{H H}^{H}\right)\right)
$$

where, $\rho=\frac{E_{s}}{N_{0}}$ is the average signal-to-noise (SNR) ratio at the $j$ th received branch. All the logarithms in this thesis are taken to base-2 so that unit of capacity takes on units of bits per channel use (also known as bits per transmission). 


\subsection{Capacity and Information Outage Probability for a Few Special Cases}

In this section we study capacity and information outage probability for a few special cases by the varying number of antennas on both sides of the link.

\subsubsection{Single-Input Single-Output Channel (SISO)}

In this case we consider single antenna at the source $\left(M_{T}=1\right)$ and single antenna at the destination $\left(M_{T}=1\right)$. We express the instantaneous capacity of the SISO system in rayleigh fading as

$$
C=\log \left(1+\rho|h|^{2}\right)
$$

where $h$ is a complex scalar. An information outage occurs when the instantaneous capacity is less than the transmission rate $R$ [bits/channeluse]. Thus information outage probability for a SISO link is given as

$$
\begin{aligned}
\mathbf{p}^{\text {outage }} & =\operatorname{Pr}(C<R) \\
& =\operatorname{Pr}\left[\log \left(1+\rho|h|^{2}\right)<R\right] \\
& =\operatorname{Pr}[\underbrace{|h|^{2}}_{Y}<\underbrace{\left.\frac{2^{R}-1}{\rho}\right]}_{z} \\
& =1-e^{-z} .
\end{aligned}
$$

where $Y$ is an exponential random variable with a parameter $\lambda=1$.

\subsubsection{Single-Input Multiple-Output Channel (SIMO)}

In this case we consider single antenna at the source $\left(M_{T}=1\right)$ and multiple antennas at the destination $\left(M_{T}=L\right)$. We express the instantaneous capacity of the SIMO system in rayleigh fading in the following manner.

$$
C=\log \left(1+\rho\|\mathbf{h}\|^{2}\right)
$$


where $\|\mathbf{h}\|^{2}=\sum_{i=1}^{L}\left|h_{i}\right|^{2}$ and $h_{i}$ is the channel gain between transmit antenna and the $i$ th receive antenna. For transmission with target rate $R$ [bits/transmission], the channel is said to be in outage if $C<R$. Hence the information outage probability is given by

$$
\begin{aligned}
& \mathbf{p}^{\text {outage }}=\operatorname{Pr}(C<R) \\
& =\operatorname{Pr}\left[\log \left(1+\rho\|\mathbf{h}\|^{2}\right)<R\right] \\
& =\operatorname{Pr}[\underbrace{\|\mathbf{h}\|^{2}}_{Y}<\underbrace{\frac{2^{R}-1}{\rho}}_{z}] \\
& =1-e^{-z} \sum_{k=0}^{L-1} \frac{z^{k}}{k !} .
\end{aligned}
$$

Here $Y$ follows a chi squared distribution with $n=2 L$ degrees of freedom. Hence we obtain the expression (2.8) in the closed form [18].

\subsubsection{Multiple-Input Single-Output Channel (MISO)}

In this case we consider single antenna at the source $\left(M_{T}=L\right)$ and multiple antennas at the destination $\left(M_{R}=1\right)$. We express the instantaneous capacity of the MISO system in rayleigh fading in the following manner.

$$
C=\log \left(1+\frac{\rho}{L}\|\mathbf{h}\|^{2}\right)
$$

where $\|\mathbf{h}\|^{2}=\sum_{i=1}^{L}\left|h_{i}\right|^{2}$ and $h_{i}$ is the channel gain between $i$ th transmit antenna and the receive antenna. Also, we can see that the total radiated power is distributed uniformly across all the transmit antennas so that the average SNR at the receiver is not a function of number of transmitters. For transmission with target rate $R$ [bits/transmission], the channel is said to be in outage if $C<R$. Hence the information outage probability is given by

$$
\begin{aligned}
\mathbf{p}^{\text {outage }} & =\operatorname{Pr}(C<R) \\
& =\operatorname{Pr}\left[\log \left(1+\frac{\rho}{L}\|\mathbf{h}\|^{2}\right)<R\right] \\
& =\operatorname{Pr}[\underbrace{\|\mathbf{h}\|^{2}}_{Y}<L \underbrace{\frac{\left(2^{R}-1\right)}{\rho}}_{z}] \\
& =1-e^{-L z} \sum_{k=0}^{L-1} \frac{(L z)^{k}}{k !} .
\end{aligned}
$$


Here $Y$ follows a chi squared distribution with $n=2 L$ degrees of freedom. Hence we obtain the expression (2.10) in a closed form [18].

\subsection{Simulation Results}

In this section we present the simulation results for outage capacity as a function of SNR obtained via a Monte Carlo simulation for various antenna array architectures. In addition, we compare information outage probability obtained using the Monte Carlo simulation and also evaluating the closed form expressions $(2.6),(2.8),(2.10)$ for the SISO, SIMO, MISO cases. The information outage probability of a MIMO channel obtained by Monte Carlo integration is shown for comparison purposes. All the simulations are carried out for a fixed spectral efficiency of $R=1$ [bits/channeluse].

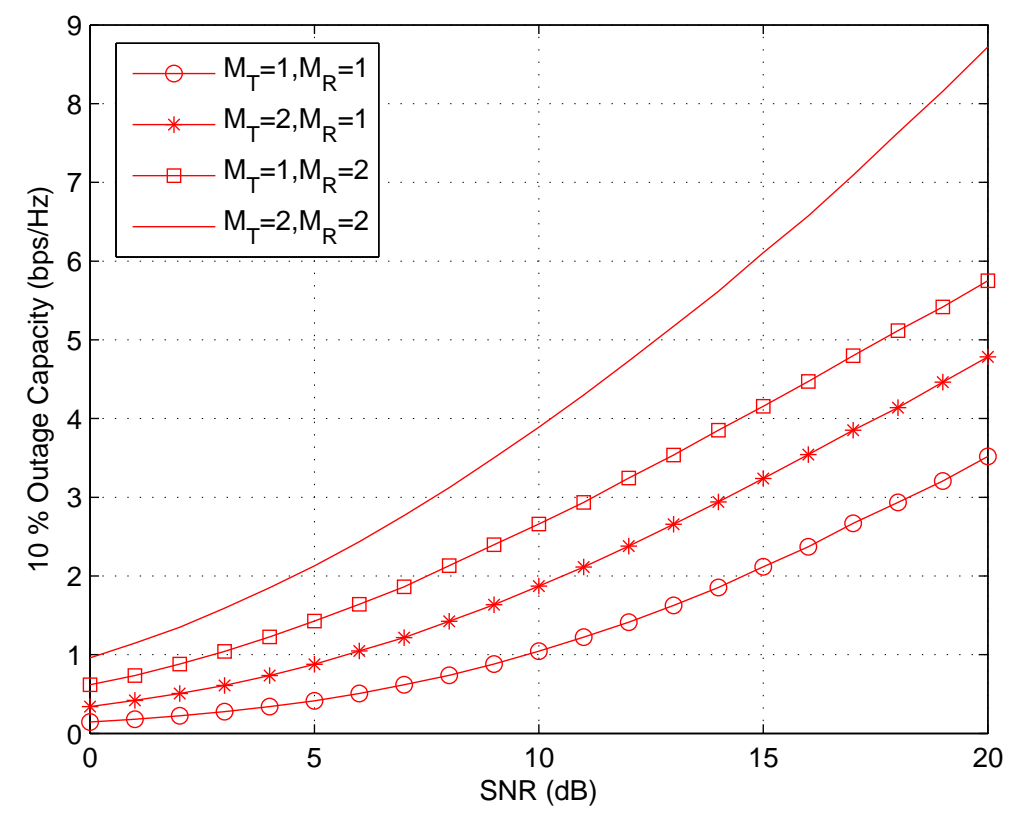

Figure 2.2: $10 \%$ Outage capacity of MIMO systems with varying antenna configurations in slowly varying rayleigh fading environment.

In 2.2 we assume a slowly varying quasi-static MIMO channel. With this assumption the notion of ergodicity is absent as the transmitted message does not experience all the fading states. Hence, we use outage capacity and information outage probability as the performance measure for such channels. In the Fig. 2.2 we compare the $10 \%$ outage capacities obtained 


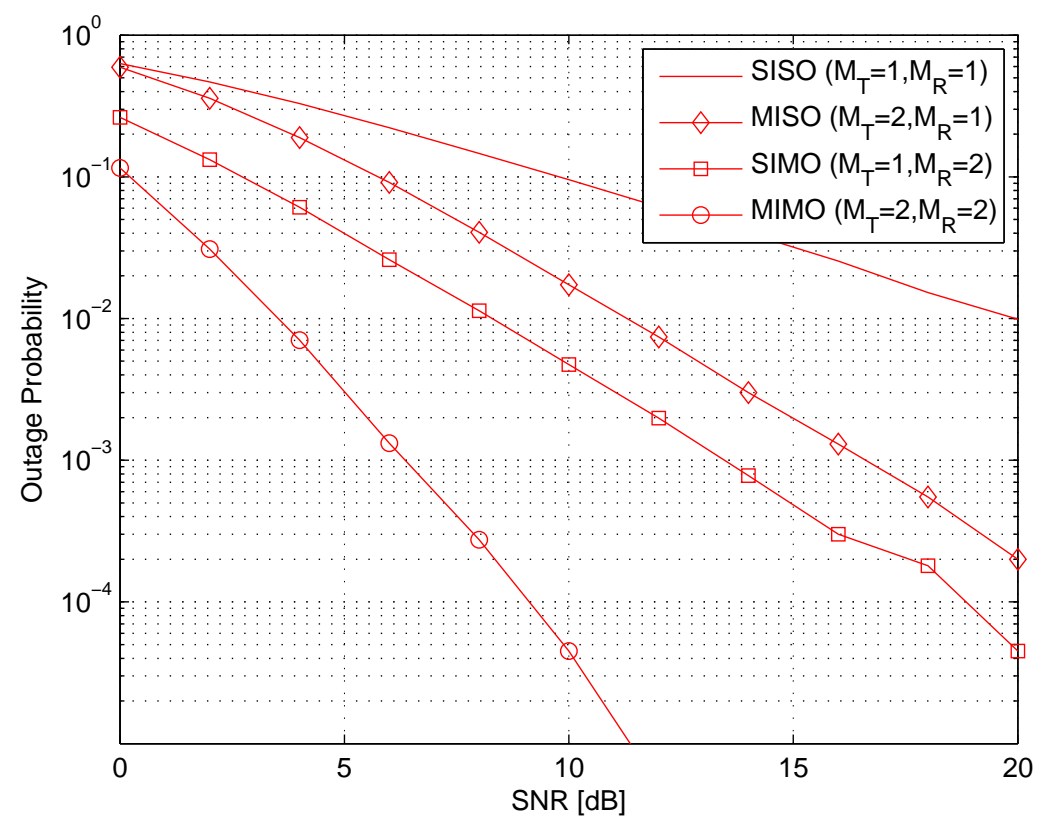

Figure 2.3: Outage Probability of MIMO systems with varying antenna configurations in slowly varying rayleigh fading environment with $R=1$ bit/channel-use.

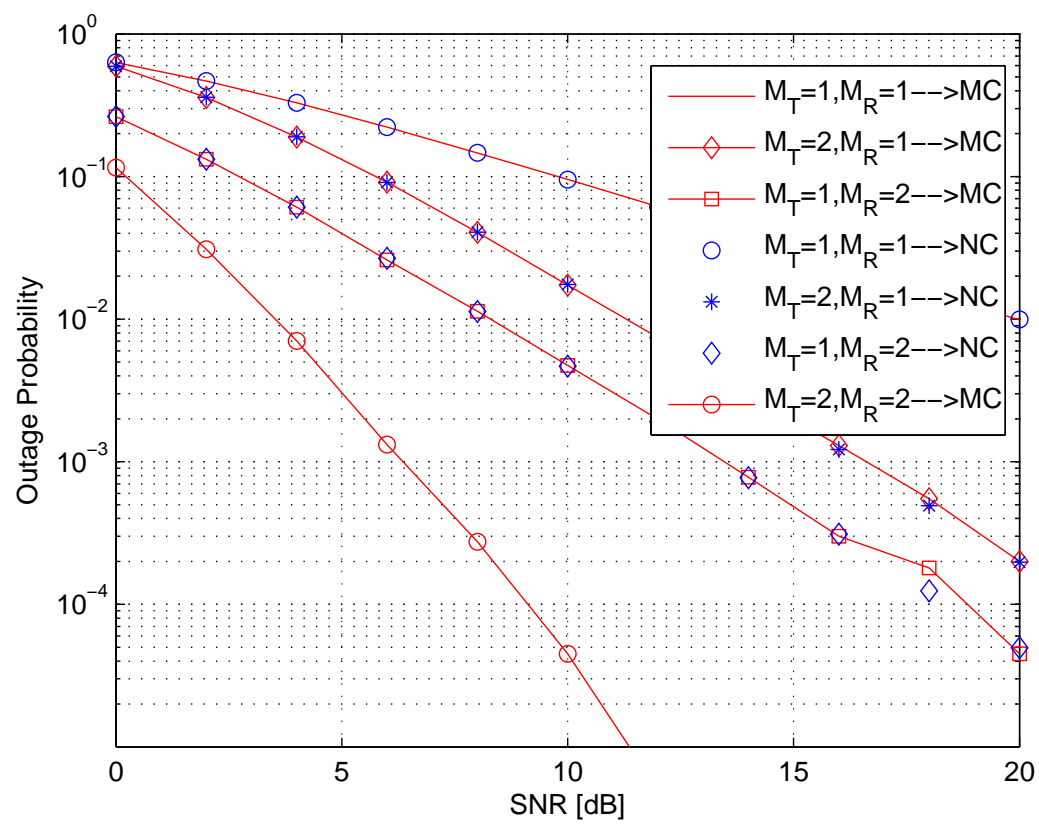

Figure 2.4: Comparison of Outage Probability of MIMO systems obtained via Monte Carlo (MC) integration method and numeric integration (NC) method for different antenna configurations in slowly varying rayleigh fading environment with $R=1$ bit/channel-use. 
using Monte Carlo integration technique for different antenna configurations. We can observe that the outage capacity of the MISO channel is always less than the SIMO channel due to the lack of channel knowledge at the transmitter. The capacity of a MIMO system is clearly dominating when compared to the capacity of MISO, SIMO and SISO systems.

Outage probability is an important statistical measure to asses the quality of the fading channels in a block fading environments. Outage specifies the probability that instantaneous channel capacity is less than the given threshold $R$ [bits/transmission]. Hence its is given in the following form $\operatorname{Pr}(C<R)$. The simulation results of information outage probability, obtained by Monte Carlo integration method for different antenna configurations in pointto-point MIMO systems is shown in Fig. 2.3. In Fig. 2.4 we compare the Monte Carlo simulated results with information outage curves obtained by numerical evaluation of the closed form expressions $(2.6),(2.8),(2.10)$. There is close match between the Monte Carlo integration method and the numerical method. We observe that there is an SNR gain in the SIMO configuration when compared to the MISO configuration due to the lack of channel knowledge at the transmitter in the MISO scheme.

\subsection{Performance of Space-Time Block Codes}

In the previous section the results obtained demonstrate the increase in the capacity limits of wireless systems by incorporating multiple antennas at both the ends of a wireless link. In addition, the information outage probability results indicate the diversity gains obtained through the use of multiple antennas. These results form a motivating factor for considering multiple antennas in a practical wireless system design. Orthogonal Space-Time Block Codes (OSTBC) are coding strategies that provide full diversity with a little or no rate penalty. In the following sections we consider a simple and novel orthogonal space time block coding technique proposed by Alamouti in [13], suited to exploit the spatial diversity gains when using two transmit antennas. The above mentioned diversity exploiting space time block code is an important aspect of consideration in the design of MIMO wireless system using the recently formulated IEEE 802.16e (WiMax) standard. 


\subsubsection{Space-Time Block Codes Using MISO System}

In the absence of channel knowledge at the transmitter, given an appropriate scheme the transmit diversity gains can be obtained. A simple and novel diversity exploiting technique by spreading the information across space and time, proposed by Alamouti in [13], is shown in Fig. 2.5. This scheme is particularly designed for wireless communication system with two transmit antennas.

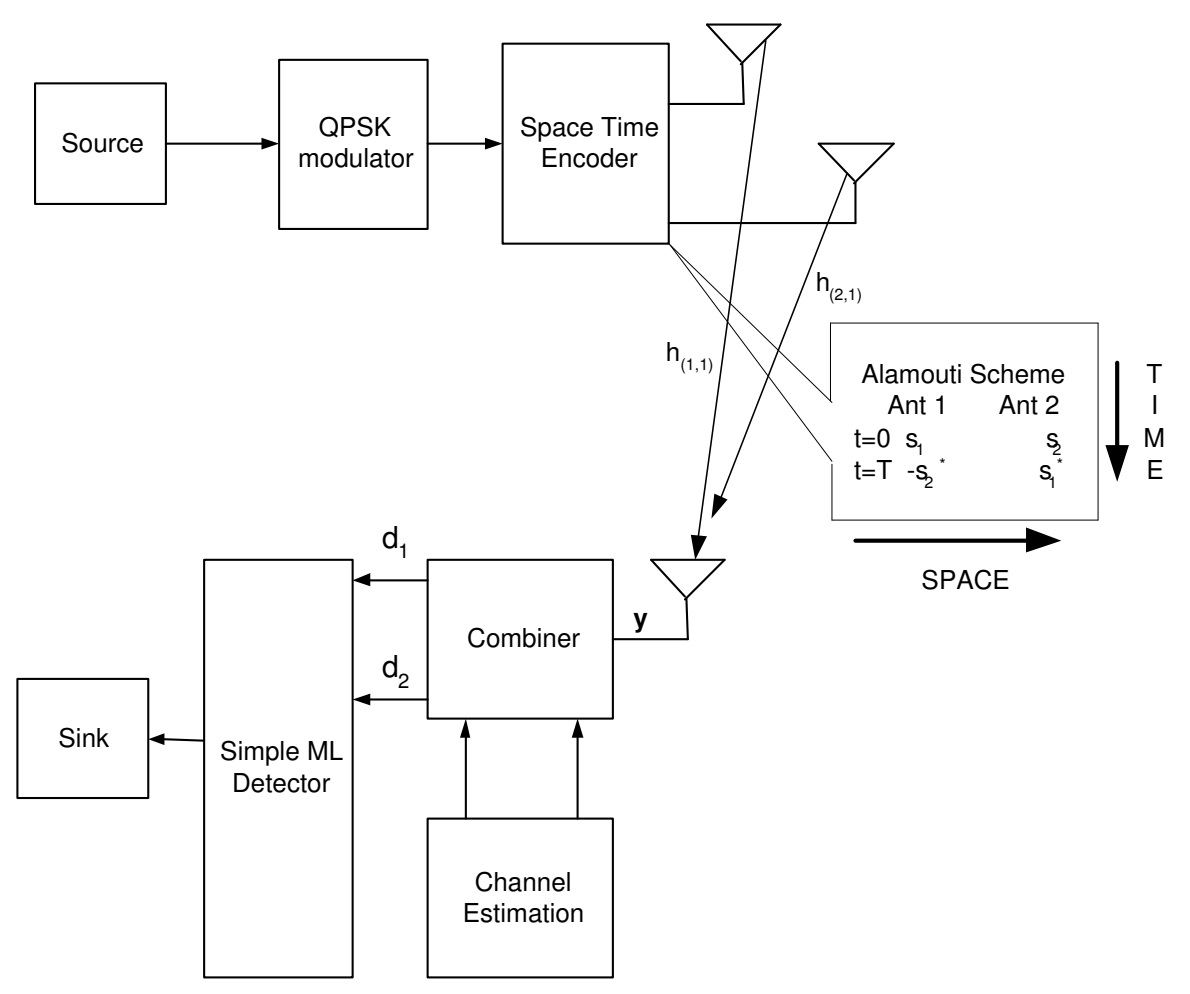

Figure 2.5: Block diagram of full rate orthogonal space time block coding scheme.

First we consider two antennas at the transmitter $\left(M_{T}=1\right)$ and a single antenna $\left(M_{R}=\right.$ 1) at the receiver. Using the Alamouti scheme, two symbols $s_{1}$ and $s_{2}$ are transmitted simultaneously from antennas 1 and 2 respectively during the first time slot. In the second time slot we transmit $-s_{2}^{*}$ and $s_{1}^{*}$ from antennas 1 and 2 respectively. Thus the Alamouti space time block encoder matrix is given by

$$
\mathbf{s}=\left[\begin{array}{cc}
s_{1} & s_{2} \\
-s_{2}^{*} & s_{1}^{*}
\end{array}\right]
$$

We assume that the channel is frequency flat and remains constant over the two symbol 
intervals.

The discrete time received signal at the received antenna after during the two symbol intervals is

$$
\begin{gathered}
y_{1}=\sqrt{\frac{E_{s}}{2}} h_{1,1} s_{1}+\sqrt{\frac{E_{s}}{2}} h_{2,1} s_{2}+n_{1}, \\
y_{2}=-\sqrt{\frac{E_{s}}{2}} h_{1,1} s_{2}^{*}+\sqrt{\frac{E_{s}}{2}} h_{2,1} s_{1}^{*}+n_{2},
\end{gathered}
$$

where $h_{i, j}$ is the complex channel gain coefficient between the transmit antenna $i$ and the receive antenna $j$ and $\frac{E_{s}}{2}$ is the average transmitted energy per symbol per antenna. The noise samples $n_{1}$ and $n_{2}$ are zero mean complex Gaussian random variables with zero mean and power $N_{o}$.

The received signal vector is formed by stacking the scalar variables as

$$
\mathbf{y}=\left[\begin{array}{ll}
y_{1} & y_{2}^{*}
\end{array}\right]^{T}
$$

Here, every other matched filer output is complex conjugated before creating the signal vector $\mathbf{y}$. Hence the received vector $\mathbf{y}$ can be expressed in matrix vector model as

$$
\mathbf{y}=\left[\begin{array}{l}
y_{1} \\
y_{2}^{*}
\end{array}\right]=\underbrace{\left[\begin{array}{cc}
h_{1,1} & h_{2,1} \\
h_{2,1}^{*} & -h_{1,1}^{*}
\end{array}\right]}_{\mathbf{H}} \underbrace{\left[\begin{array}{l}
s_{1} \\
s_{2}
\end{array}\right]}_{\mathbf{s}} \sqrt{\frac{E_{s}}{2}}+\underbrace{\left[\begin{array}{c}
n_{1} \\
n_{2}^{*}
\end{array}\right]}_{\mathbf{n}}
$$

This matrix-vector model is different from (2.1) and is, in itself not interesting. At the receiver we match filter with respect to the channel forming the decision static

$$
\begin{aligned}
\mathbf{d} & =\mathbf{H}^{H} \mathbf{y} \\
& =\mathbf{H}^{H} \mathbf{H} \mathbf{s} \sqrt{\frac{E_{s}}{2}}+\mathbf{H}^{H} \mathbf{n} \\
& =\left[\begin{array}{cc}
E_{h} & 0 \\
0 & E_{h}
\end{array}\right] \mathbf{s} \sqrt{\frac{E_{s}}{2}}+\underbrace{\mathbf{H}^{H} \mathbf{n}}_{\nu}
\end{aligned}
$$

here

$$
E_{h}=\sum_{i=1}^{M_{T}} \sum_{j=1}^{M_{R}}\left|h_{i, j}\right|^{2}
$$

and $\nu \sim \mathcal{N}_{c}\left(\mathbf{0}, E_{h} N_{o} \mathbf{I}\right)$. 
The decision static of $s_{1}$ is not contaminated with decision static of $s_{2}$. This is due to the diagonal nature of $\mathbf{d}$, i.e. the matrix $\mathbf{H}^{H} \mathbf{H}$ is diagonal, which effectively decouples the individual symbol transmissions. Hence each component of $\mathbf{d}$ corresponds to one of the transmitted symbols:

$$
\begin{aligned}
& d_{1}=\left[\left|h_{1,1}\right|^{2}+\left|h_{2,1}\right|^{2}\right] s_{1} \sqrt{\frac{E_{s}}{2}}+\nu_{1}, \\
& d_{2}=\left[\left|h_{1,1}\right|^{2}+\left|h_{2,1}\right|^{2}\right] s_{2} \sqrt{\frac{E_{s}}{2}}+\nu_{2},
\end{aligned}
$$

The instantaneous SNR for each symbol is

$$
\mathrm{SNR}=\frac{\left(\left|h_{1,1}\right|^{2}+\left|h_{2,1}\right|^{2}\right) E_{s}}{2 N_{0}}
$$

which is same as the instantaneous SNR for a two branch maximal ratio combining scheme (MRC). The system provides the same diversity gain as that of a MRC (2) scheme although there is a $3-\mathrm{dB}$ loss in the SNR caused due to the power division across the two transmit antennas. The maximum likelihood (ML) estimates are given by

$$
\begin{gathered}
\tilde{s_{1}}=\underset{s \epsilon \chi}{\operatorname{argmin}}\left|d_{1}-s\right|^{2}, \\
\tilde{s_{2}}=\underset{s \epsilon \chi}{\operatorname{argmin}}\left|d_{2}-s\right|^{2},
\end{gathered}
$$

where $\chi$ is the symbol alphabet. The ML estimates are same as that of the ML estimates in white noise. Hence no equalization is required. We can also see that there is no sacrifice in the bandwidth as two symbols are transmitted over two time intervals. This system thus operates at full rate.

\subsubsection{Space-Time Block Codes Using MIMO Systems}

Here we consider two $M_{T}=2$ transmit antennas and $M_{R}=2$ receive antennas. Transmitting the same Alamouti space-time code word the discrete time received signal at antenna 1 after the two transmissions is

$$
\begin{gathered}
y_{1}^{1}=\sqrt{\frac{E_{s}}{2}} h_{1,1} s_{1}+\sqrt{\frac{E_{s}}{2}} h_{2,1} s_{2}+n_{1}^{1}, \\
y_{2}^{1}=-\sqrt{\frac{E_{s}}{2}} h_{1,1} s_{2}^{*}+\sqrt{\frac{E_{s}}{2}} h_{2,1} s_{1}^{*}+n_{2}^{1},
\end{gathered}
$$


and the received signals at antenna 2 are

$$
\begin{gathered}
y_{1}^{2}=\sqrt{\frac{E_{s}}{2}} h_{1,2} s_{1}+\sqrt{\frac{E_{s}}{2}} h_{2,2} s_{2}+n_{1}^{2}, \\
y_{2}^{2}=-\sqrt{\frac{E_{s}}{2}} h_{1,2} s_{2}^{*}+\sqrt{\frac{E_{s}}{2}} h_{2,2} s_{1}^{*}+n_{2}^{2},
\end{gathered}
$$

The received vector is formed by stacking the received signals is expressed as

$$
\mathbf{y}=\left[\begin{array}{c}
y_{1}^{1} \\
y_{2}^{1^{*}} \\
y_{1}^{2} \\
y_{2}^{2^{*}}
\end{array}\right]=\underbrace{\left[\begin{array}{cc}
h_{1,1} & h_{2,1} \\
h_{2,1}^{*} & -h_{1,1}^{*} \\
h_{1,2} & h_{2,2} \\
h_{2,2}^{*} & -h_{1,2}^{*}
\end{array}\right]}_{\mathbf{H}} \underbrace{\left[\begin{array}{c}
s_{1} \\
s_{2}
\end{array}\right]}_{\mathbf{s}} \sqrt{\frac{E_{s}}{2}}+\underbrace{\left[\begin{array}{c}
n_{1}^{1} \\
n_{2}^{1^{*}} \\
n_{1}^{2} \\
n_{2}^{2^{*}}
\end{array}\right]}_{\mathbf{n}}
$$

The following decision static obtained after we match filter with respect to the channel

$$
\begin{aligned}
\mathbf{d} & =\mathbf{H}^{H} \mathbf{y} \\
& =\mathbf{H}^{H} \mathbf{H} \mathbf{s} \sqrt{\frac{E_{s}}{2}}+\mathbf{H}^{H} \mathbf{n} \\
& =\left[\begin{array}{cc}
E_{h} & 0 \\
0 & E_{h}
\end{array}\right] \mathbf{s} \sqrt{\frac{E_{s}}{2}}+\underbrace{\mathbf{H}^{H} \mathbf{n}}_{\nu}
\end{aligned}
$$

here

$$
E_{h}=\sum_{i=1}^{M_{T}} \sum_{j=1}^{M_{R}}\left|h_{i, j}\right|^{2}
$$

and $\nu \sim \mathcal{N}_{c}\left(\mathbf{0}, E_{h} N_{o} \mathbf{I}\right)$.

Thus the elements of vector $\mathbf{d}$ are

$$
\begin{aligned}
& d_{1}=\left[\left|h_{1,1}\right|^{2}+\left|h_{2,1}\right|^{2}+\left|h_{1,2}\right|^{2}+\left|h_{2,2}\right|^{2}\right] s_{1} \sqrt{\frac{E_{s}}{2}}+\nu_{1}, \\
& d_{2}=\left[\left|h_{1,1}\right|^{2}+\left|h_{2,1}\right|^{2}+\left|h_{1,2}\right|^{2}+\left|h_{2,2}\right|^{2}\right] s_{2} \sqrt{\frac{E_{s}}{2}}+\nu_{2},
\end{aligned}
$$

Hence we find that the instantaneous SNR for each symbol is

$$
\mathrm{SNR}=\frac{\left(\left|h_{1,1}\right|^{2}+\left|h_{1,2}\right|^{2}+\left|h_{2,1}\right|^{2}+\left|h_{2,2}\right|^{2}\right) E_{s}}{2 N_{0}}
$$

which is same as the instantaneous SNR for a four branch MRC. Thus the system provides a diversity order same as that of a MRC with four branches with a $3 \mathrm{~dB}$ penalty in SNR. 


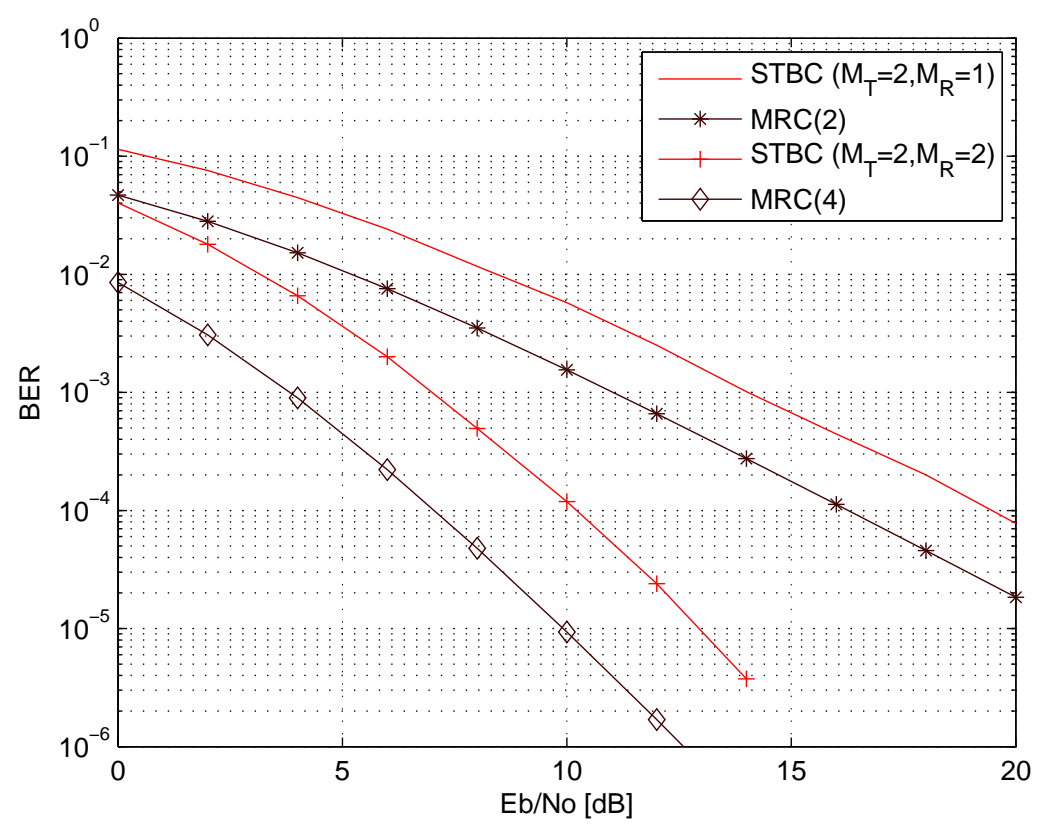

Figure 2.6: Bit error rate of orthogonal space time block code with $\mathrm{R}=2$ bits/channel-use.

\subsubsection{Simulation Results}

The performance of the orthogonal space-time code proposed by Alamouti is presented in terms of bit error rate (BER) for configurations $M_{T}=2, M_{R}=1,2$ is shown in Fig. 2.6. We assume a QPSK modulation scheme and the energy of each symbol is given as $E_{s}=2 E_{b}$ with unit bit energy i.e. $E_{b}=1$. The BER plot with receive antenna $M_{R}=1$ is compared with the maximal ratio combining scheme with $M_{R}=2$ branches. The plots indicate that the diversity gain is same as that of the MRC with 2 branches. The performance with $M_{R}=2$ is compared with MRC with 4 branch reception. The slope of the curve is same as that of MRC indicating full diversity. Although, in both the cases there is a 3dB SNR loss due to the power splitting across the two transmit antennas.

Fig. 2.7 indicates the normalized throughput performance of the using space time block codes proposed by Alamouti with $M_{T}=2, M_{R}=1,2$. Normalized throughput, $T$, is calculated as

$$
T=R(1-\mathrm{BER})^{M} \mathrm{bits} / \text { channel-use, }
$$

For transmission using the orthogonal $\operatorname{STBC} T=T_{s t b c}\left(0 \leq T_{s t b c} \leq 2\right)$ is the normalized 


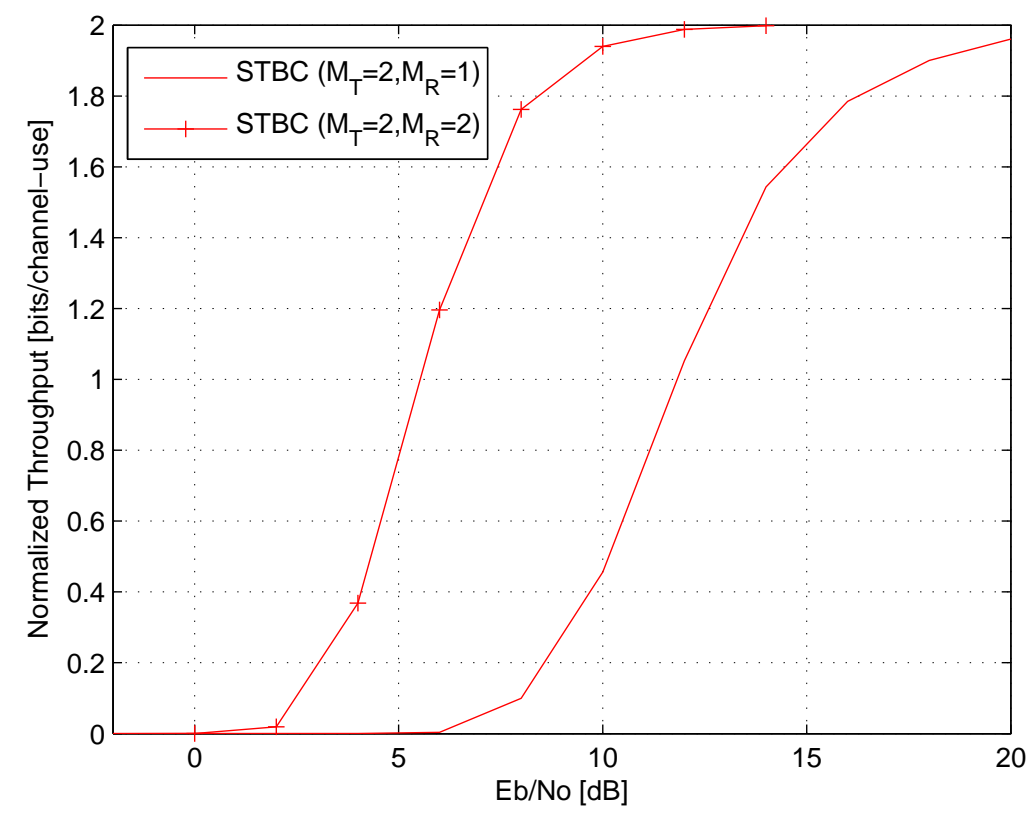

Figure 2.7: Normalized Throughput of orthogonal space time block code.

throughput, $R=R_{s t b c}$ is the normalized rate and $M=256$ is the packet size. The transmit diversity scheme operates at rate $R_{s t b c}=2$. The throughput performance of multiple antenna $M_{R}=2$ reception scheme is compared against the single antenna reception $M_{R}=1$, for the same number of transmit antennas using the same space-time code word. We can observe at normalized thruput of 1 bit/channel-use, there is an SNR gain of $7 \mathrm{~dB}$ during the multi antenna reception when compared with the single antenna reception scheme.

\subsection{Performance Analysis of BLAST}

In the previous section we analyze the performance of space time block code proposed by Alamouti in [13]. The orthogonal space time codes are used to achieve high orders of diversity with a little penalty in the data rate. This penalty in terms of data rate does not achieve full capacity limits of a MIMO channel. The actual capacity limits were explored by Foschini and Gans in [1] from a information-theoretic stand point. In order to realize these capacity limits Foschini in [19] propose Bell LAbs Layered Space-Time (BLAST) architecture for rayleigh fading environments. In [20] Woliniansky et.al. propose a more practically realizable and a 
modified scheme called the vertical BLAST or V-BLAST. The BLAST architecture utilizes multiple antennas at both ends of a wireless link to support very high data rate when compared with single antenna wireless system. The signal processing at the receiver is based on a divide-and-conquer technique, instead of performing joint decoding, where the signals from multiple antennas are treated as interference and cancelled using nulling (beamforming) and interference cancellation techniques. The nulling criteria considered in the literature are the Zero Forcing (ZF) and Minimum-Mean Squared Error (MMSE) criterion. Although a high diversity order is expected due to the use of multiple antennas, the error propagation mechanism that occurs during the successive interference cancellation (SIC) accounts for the inferior performance and has been demonstrated in [21], [22].

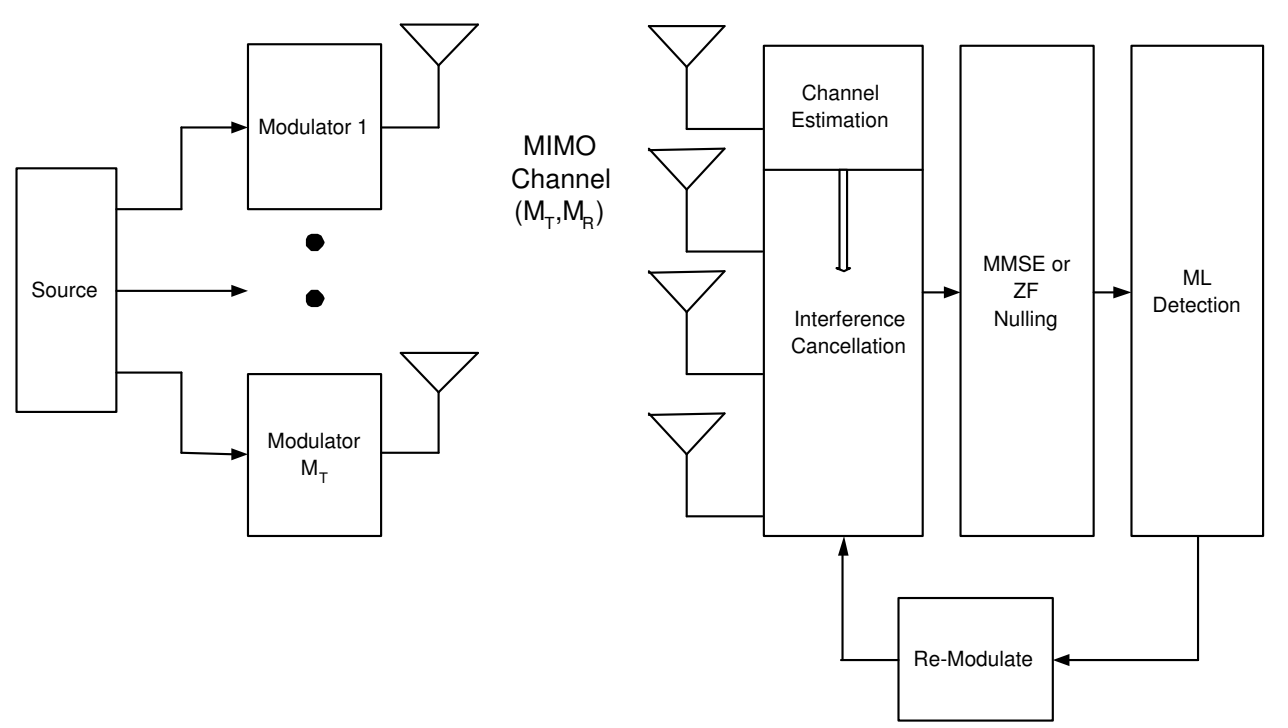

Figure 2.8: Block diagram of V-BLAST architecture.

A block diagram of VBLAST is shown in Fig. 2.8. In this scheme the serially generated information symbols are mapped into parallel streams and each parallel stream is transmitted from one transmit antenna. Assuming a rich scattering channel and reasonable antenna spacing, the fading across the transmit antennas will be independent. The channel is assumed to be quasi-static Rayleigh flat fading channel. Assuming $M_{T}$ is the number of transmit antennas and $M_{R}$ is the number of receive antennas, we recall the discrete-time received 
signal vector modeled in 2.1 as

$$
\mathbf{r}=\sqrt{\frac{E_{s}}{M_{T}}} \mathbf{H} \mathbf{s}+\mathbf{n}
$$

where the dimension of the received vector is $M_{R} \times 1$. $\mathbf{H}$ represents the $M_{R} \times M_{T}$ channel matrix representing the MIMO quasi-static flat fading channel between the transmitter and receiver. $\mathbf{s}$ is a $M_{T} \times 1$ vector of transmitted symbols. $\mathbf{n}$ is a $M_{R} \times 1$ complex vector of independent AWGN noise samples with zero mean and variance $\frac{N_{o}}{2}$ per complex dimension. We assume a QPSK modulation scheme with unit bit energy and $M_{T}=2, M_{R}=2$, hence $\frac{E_{s}}{M_{T}}=1$.

We implement a non linear reception scheme at the receiver and it is called as successive interference cancellation (SIC) receiver. The main idea behind this scheme is that the decoding is performed using a layered approach. Detection of the signals can be viewed as a three step procedure. We assume a perfect channel estimation at the receiver.

1. Nulling: The receiver initially estimates the channel matrix $\mathbf{H}$. The receiver, while detecting the $i$ th symbol from a particular antenna, considers all the other symbols as interferers. Then it uses a nulling matrix based on the zero forcing (ZF) or the minimum mean squared error criterion (MMSE) to suppress the signals from other antennas. The nulling matrix for zero forcing is given as $\mathbf{G}=\left(\mathbf{H}^{H} \mathbf{H}\right) \mathbf{H}^{H}$. The nulling matrix for the MMSE criteria is given by $\mathbf{G}=\left(\mathbf{H}^{H} \mathbf{H}+\mathrm{SNR}^{-1} I_{M_{T}}\right) \mathbf{H}^{H}$. In order to obtain the $i$ th signal transmitted from the transmit antenna $i$, we multiply the received signal with the weight vector given by $w_{i}=(\mathbf{G})_{i}^{H}$. Here $(\mathbf{G})_{i}^{H}$ is the $i$ th column of the nulling matrix $\mathbf{G}$.

2. Slicing: The decision static $y_{i}$ is obtained as follows, $y_{i}=w_{i}^{T} \mathbf{r}$. An ML estimate $\tilde{s}_{i}=$ $Q\left(y_{i}\right)$ is obtained from $y_{i}$. Here $Q()$ represents the slicing operation or quantization.

3. Cancellation: In this step assuming the estimated symbol is correct we cancel the effect of this symbol from the received signal vector. The updated received symbol is given by $r_{i+1}=r_{i}-\tilde{s}_{i} \mathbf{H}_{(:, i)}$. The column of $\mathbf{H}$ corresponding to the symbol most recently detected is removed and nulling matrix is recalculated. 
This process is repeated until all the transmitted symbols are detected. The above process does not include ordering of symbol stream and hence we start our decoding process by choosing the symbol stream from the first transmit antenna. It was shown in [20] optimal ordered successive cancellation based on the instantaneous signal-to-noise ratio yield better results. The purpose of ordering is to decide upon which transmitted symbol stream has to be decoded at each stage of decoding. Hence during ordering, we decode the strongest symbol stream i.e. the symbol stream with highest instantaneous SNR. After the ordering step we then perform the interference cancellation stage by stage as described before.

The performance of BLAST is limited by the accuracy of the symbols detected in the first stage. Assuming ideal cancellation, the diversity order should increase with each stage of detection. For a particular stage the receiver with $M_{R}$ antennas should suppress the signals from $M_{T}-1$ transmit antennas. This was found to equivalent to a system with one transmit and $M_{R}-M_{T}+1$ receive antennas in [23]. As the cancellation is performed stage by stage, any errors in the decision after first stage propagates to the later stages. This is called error propagation in BLAST. These effects were extensively studied and some novel methods using iterative cancellation techniques at the receiver side was proposed to mitigate the effects of error propagation in [21].

\subsection{Simulation Results}

The performance of BLAST with ZF, MMSE nulling methods is presented in Fig. 2.9 for $M_{T}=2, M_{R}=2$. The MMSE nulling leads to an improved performance when compared to $\mathrm{ZF}$ nulling operation in the low SNR regime. This is due to the fact that the ZF nulling operation removes all the interference at the cost of increasing background noise. In the high SNR the ZF criterion has a similar performance when compared to MMSE nulling criterion. Two different aspects of successive interference cancellation methods is performed using ZF, MMSE nulling methods. The first method is successive interference cancellation detection without ordering of signals, the second method is to perform detection by ordered successive interference cancellation based on the highest post detection SNR. The Fig. 2.9 indicates that ordering of signals improves the performance of BLAST reception. At a BER of $10^{-3}$ 


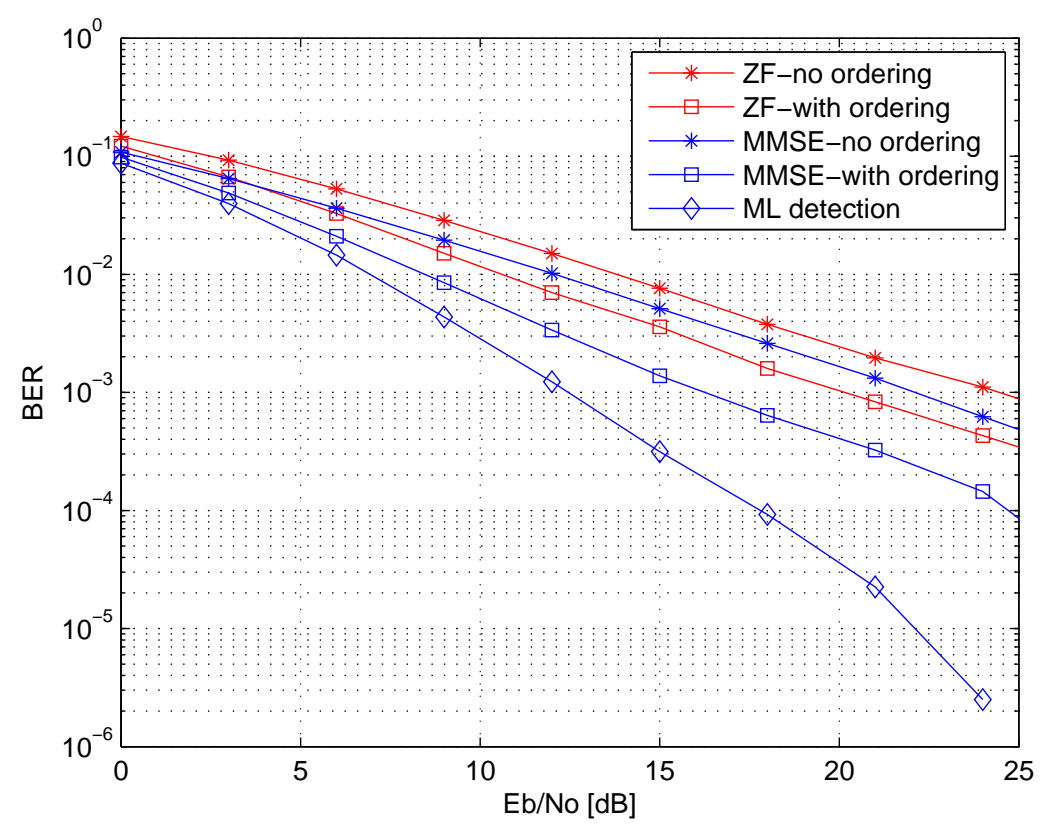

Figure 2.9: Bit error rate of VBLAST for $M_{T}=2, M_{R}=2$ in rayleigh fading with $\mathrm{R}=4$ bits/channel-use.

we can observe that the performance of MMSE detection using ordering has an SNR gain of $4 \mathrm{~dB}$ when compared to the performance of a ZF nulling with ordered detection. When compared against of the ML detection scheme we observe the SIC receiver does not achieve the full diversity gains due to error propagation as studied in [21].

The Fig. 2.10 represents the normalized throughput is calculated using equation (2.35), where the normalized throughput is $T=T_{\text {vblast }}\left(0 \leq T_{\text {vblast }} \leq 4\right)$, the normalized rate $R=R_{\text {vblast }}=4$ and $M=256$ is the packet size. We can observe that the throughput obtained using the MMSE criterion has a better performance when compared to the ZF counterpart. Also, the ordered SIC using MMSE nulling scheme has a performance gain of 6dB SNR than the MMSE nulling without ordered detection. The ZF nulling with ordering has a gain of $3 \mathrm{~dB}$ in SNR when compared to ZF nulling without ordering. 


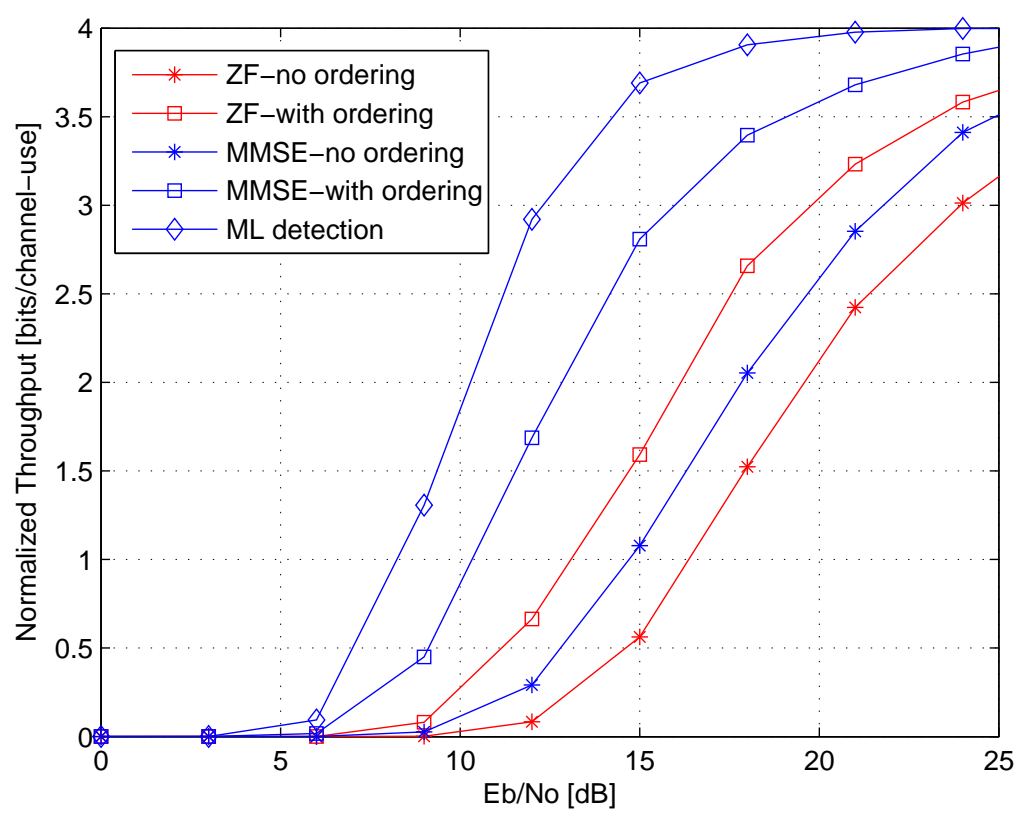

Figure 2.10: Normalized throughput of VBLAST for $M_{T}=2, M_{R}=2$ in rayleigh fading.

\subsection{Chapter Summary}

This chapter initially presents information theoretic overview of MIMO wireless channel. Results of capacity analysis, presented in terms of ergodic capacity and information outage probability highlight the achievable rates and diversity gains offered due to incorporation of multiple antennas at both the ends of a wireless link. Further we present the practical schemes using Alamouti space time code word to exploit the diversity gains in MIMO systems [13]. Also, we realize the maximum achievable rates in a practical MIMO wireless system by a simulation study of VBLAST architecture proposed in [1]. Results presented in terms of BER an throughput indicate the advantages of MIMO wireless systems in Rayleigh fading environments. In the next chapter we study the impact of antenna arrays in relay networks using the closed from expressions obtained for various cases in a relay network setting. 


\section{Chapter 3}

\section{Impact of Multiple Antennas on Cooperative Relay Networks}

In this chapter we consider the impact of the presence of antenna arrays in the relay network with a single relay assuming two different combining schemes, namely, diversity combining and code combining at the destination. First, we focus on the adaptive decodeand-forward (DF) protocol with both the combining schemes individually, whereby the relay retransmits only if it successfully decodes the source message (under the assumption of a perfect error correction code) and study the impact of single antenna array which may be placed at the source, relay or destination. Second, we study the impact of the antenna arrays by allowing the antenna array to be placed at more than one terminal simultaneously. An information theoretic approach is taken for both the cases, and our main aim is to quantify the performance in terms of information outage probability.

In the first case, by limiting the array to be placed at only one node assuming code combining scheme at destination, when the array is placed at the source and relay, a onedimensional integral is obtained which can be solved numerically, while at the destination we obtain the results using Monte Carlo integration method. In the diversity combining assumption for the first case, when the array is at the destination, a closed form solution is obtained, while for the cases that the antenna array is at the relay or the source, the expression contains a one-dimensional integral which can be solved numerically. This analysis gives us an insight into the possible location of the antenna array when we have a constraint 


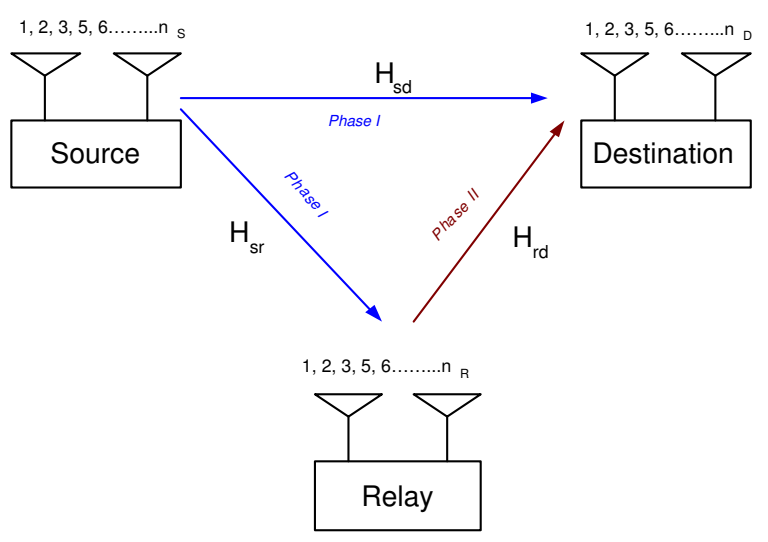

Figure 3.1: A three terminal relay network.

to locate all the additionally available antennas at a particular node after assigning one antenna to each of the three nodes in a relay network. In the second case, we relax our constraint by locating the antenna array at more than one node simultaneously to find out the optimal distribution of antennas in a relay network. Due to the analytical complexity with increasing antennas, we perform the analysis using Monte Carlo integration for both code combining scheme and diversity combining scheme in the latter case.

\subsection{Relay Networks}

Consider the three terminal network shown in Fig. 3.1. Let $n_{S}, n_{R}$, and $n_{D}$ denote the number of antennas at the source, relay, and destination, respectively. The source and relay transmit with identical power, and if a particular terminal uses multiple transmit antennas, the power is divided across that terminal's antennas. To remove the effects of topology, we assume that the path loss between terminals is identical, which is true if the three terminals are located at the corners of an equilateral triangle. The channels between nodes are independent, and each is a quasi-static Rayleigh flat fading channel. Communication takes place through two time slots of equal duration. In the first slot, the source transmits and both relay and destination listen. In the second slot, the relay will retransmit the initial source message, but only if the relay successfully decoded the source's initial transmission (c.f. adaptive $\mathrm{DF})$. 
The signal at the relay due to the source transmission is

$$
\mathbf{y}_{s r}=\sqrt{\frac{E_{s}}{n_{S}}} \mathbf{H}_{s r} \mathbf{s}_{s}+\mathbf{n}_{r}
$$

while the signal at the destination due to the source transmission is

$$
\mathbf{y}_{s d}=\sqrt{\frac{E_{s}}{n_{S}}} \mathbf{H}_{s d} \mathbf{s}_{s}+\mathbf{n}_{d_{1}}
$$

where $\mathbf{H}_{s r}$ is the $n_{R} \times n_{S}$ channel gain matrix for the source-relay channel, $\mathbf{H}_{s d}$ is the $n_{D} \times n_{S}$ channel gain matrix for the source-destination channel, $\mathbf{s}_{s}$ is a length $n_{S} \times 1$ transmitted signal vector, and $\mathbf{n}_{r}, \mathbf{n}_{d_{1}}$ are the respective channel noise vectors drawn from an ensemble of i.i.d complex Gaussian random variables with zero mean and variance $N_{o}$.

If the relay decodes correctly, it retransmits the message to the destination during the second slot. In repetition based cooperation (diversity combining) the relay retransmits exactly the same message as that of the source. In case of coded cooperation (code combining) the relay may retransmits a different codeword. The signal at the destination due to the relay transmission is

$$
\mathbf{y}_{r d}=\sqrt{\frac{E_{s}}{n_{R}}} \mathbf{H}_{r d} \mathbf{s}_{r}+\mathbf{n}_{d_{2}}
$$

where $\mathbf{H}_{r d}$ is the $n_{D} \times n_{R}$ channel gain matrix of the of the relay-destination path, $\mathbf{s}_{r}$ is a length $n_{R} \times 1$ transmitted signal vector, and $\mathbf{n}_{d_{2}}$ is a vector of i.i.d. Gaussian noise vector with zero mean and variance $N_{o}$. The source remains silent during the second slot.

\subsection{Relay Network Outage Analysis: Code Combining}

Code combining can be performed by assuming that the message transmitted from the source and the relay is generated using entirely independent Gaussian codebooks. This is also known is also known as parallel channel coding [24]. A more practical approach to perform code combining is assumed and is explained as follows: The source creates a rate $R$ codeword of length $N$ but only transmits a fraction $\alpha$ of the coded symbols as a $\alpha N$ bit sequence. The relay receives and decodes the symbols. If the sequence is decoded correctly then the relay will rencode with the same rate $R$ code, but will transmit the $(1-\alpha) N$ code 
symbols that were not transmitted by the source. The destination receives and decodes the entire $N$ bit codeword. The instantaneous capacity of the system assuming code combining at destination is given in [12] by the following expression

$$
C_{D F-C C}=\max \left\{\min \{\alpha C_{s d}+\underbrace{(1-\alpha)}_{\bar{\alpha}} C_{r d}, \alpha C_{s r}\}, \alpha C_{s d}\right\}
$$

where, $C_{s d}$ is the instantaneous capacity of the source-destination (S-D) path, $C_{r d}$ is the instantaneous capacity of relay-destination (R-D) path, and $\alpha C_{s d}+\bar{\alpha} C_{r d}$ is the sum of the instantaneous capacities assuming code combining at destination from S-D and R-D paths. In [24], a similar expression was found for distributed space-time coding, which requires $\alpha=0.5$. An outage occurs when the instantaneous capacity (3.4) is less than the transmission rate $R$ i.e. $C_{D F-C C}<R$. The equivalent outage event is given as:

$$
\left[\left(C_{s r}<\frac{R}{\alpha}\right) \cap\left(C_{s d}<\frac{R}{\alpha}\right)\right] \cup\left[\left(C_{s r}>\frac{R}{\alpha}\right) \cap\left(\alpha C_{s d}+\bar{\alpha} C_{r d}<R\right)\right]
$$

As the events in (3.5) are mutually exclusive, the information outage probability becomes a sum and is given as

$$
\begin{aligned}
\mathbf{p}_{C C}^{\text {outage }} & =\operatorname{Pr}\left(C_{D F-C C}<R\right) \\
& =\operatorname{Pr}\left(C_{s r}<\frac{R}{\alpha}\right) \operatorname{Pr}\left(C_{s d}<\frac{R}{\alpha}\right)+\operatorname{Pr}\left(C_{s r}>\frac{R}{\alpha}\right) \operatorname{Pr}\left(\alpha C_{s d}+\bar{\alpha} C_{r d}<R\right)
\end{aligned}
$$

We now evaluate this expression for three cases of interests similar to the diversity combining schemes with the array at the destination, relay and source respectively. Also for the purposes of evaluation we assume $\alpha=0.5$ initially. In section 3.6 we show that this value is suboptimal and present optimal values of $\alpha$ for different antenna array configurations in case of codecombining scheme.

\subsubsection{Array at the Source}

In this configuration, the single antenna relay node assists the multi-antenna antenna source node and single antenna destination node (i.e. $n_{S}=L, n_{R}=1, n_{D}=1$ ). In this case, $\mathbf{H}_{s d}=\mathbf{h}_{s d}$ and $\mathbf{H}_{s r}=\mathbf{h}_{s r}$ are length $L$ row vectors (S-D and S-R are MISO), and $\mathbf{H}_{r d}=h_{r d}$ is scalar (R-D is SISO). The information outage probability for the S-D channel is same as 
the MISO channel with $L$ transmit antennas with $z=\left(2^{\frac{R}{\alpha}}-1\right) / \rho$, where $\rho=\frac{E_{s}}{N_{o}}$ as defined in Chapter 2.

$$
\operatorname{Pr}\left(C_{s d}<\frac{R}{\alpha}\right)=1-e^{-L z} \sum_{k=0}^{L-1} \frac{(L z)^{k}}{k !} .
$$

The information outage probability for the S-R channel is also that of an MISO channel with $L$ transmit antennas

$$
\operatorname{Pr}\left(C_{s r}<\frac{R}{\alpha}\right)=1-e^{-L z} \sum_{k=0}^{L-1} \frac{(L z)^{k}}{k !} .
$$

The term $\operatorname{Pr}\left(\alpha C_{s d}+\bar{\alpha} C_{r d}<R\right)$ represents the information outage probability due to the code combining at destination from the SISO path S-D and SIMO path R-D. The information outage is given as follows.

$$
\begin{aligned}
\operatorname{Pr}\left(\alpha C_{s d}+\bar{\alpha} C_{r d}<R\right) & =\operatorname{Pr}\left[\alpha \log (1+\rho \underbrace{\frac{\left\|\mathbf{h}_{s d}\right\|^{2}}{L}}_{X})+\bar{\alpha} \log (1+\rho \underbrace{\left|h_{r d}\right|^{2}}_{Y})<R\right] \\
& =\operatorname{Pr}\left[(1+X \rho)^{\alpha}(1+Y \rho)^{\bar{\alpha}}<2^{R}\right] \\
& =\int_{0}^{b} \int_{0}^{a} f_{x}(x) f_{y}(y) d y d x
\end{aligned}
$$

where, $a=\rho^{-1}\left(\frac{2^{\frac{R}{\alpha}}}{(1+x \rho)^{\frac{\alpha}{\alpha}}}-1\right)$. and $b=z$.

The expression (3.9) is obtained due to independence of random variables $X, Y$ following a similar simplification given in [7]. Here $X, Y$ are independent random variables. $X$ follows a $\chi^{2}$ distribution with $n_{1}=2 L$ degress of freedom (DOF), while $Y$ is exponentially distributed with parameter $\lambda=1$ or equivalently $\chi^{2}$ with 2 DOF. The individual pdf's are given by $f_{X}(x)=\frac{L(L x)^{L-1} e^{-L x}}{(L-1) !} u(x)$, is the scaled version of the $\chi^{2}$ pdf [18] with factor $L$, and $f_{Y}(y)=$ $e^{-y} u(y)$, where $u(x)$ is a unit step function. Substitution in (3.9) and further integrating over $a, b$ yields

$$
\begin{aligned}
\operatorname{Pr}\left(\alpha C_{s d}+\bar{\alpha} C_{r d}<R\right) & =\int_{0}^{z} \int_{0}^{a} \frac{(L x)^{L-1} e^{-L x}}{(L-1) !} e^{-y} d y d x \\
& =\frac{L^{L}}{(L-1) !} \int_{0}^{z} x^{L-1} e^{-L x}\left[1-e^{-a}\right] d x
\end{aligned}
$$

Substituting (3.7), (3.8), and (3.10) into (3.6), we arrive at the following expression for information outage probability for this case (i.e. $n_{S}=L, n_{R}=1, n_{D}=1$ ) assuming code 
combining at the destination.

$$
\begin{aligned}
\mathbf{p}_{(L, 1,1)-C C}^{\text {outage }} & =\left[\left(1-e^{-L z} \sum_{k=0}^{L-1} \frac{(L z)^{k}}{k !}\right)^{2}\right. \\
& \left.+\left(e^{-L z} \sum_{k=0}^{L-1} \frac{(L z)^{k}}{k !}\right)\left(\frac{L^{L}}{(L-1) !} \int_{0}^{z} x^{L-1} e^{-L x}\left[1-e^{-a}\right] d x\right)\right] .
\end{aligned}
$$

\subsubsection{Array at the Relay}

In this section we perform outage analysis of a simple three terminal relay network with single antennas at source, relay and destination with multiple antennas (i.e. $n_{S}=1, n_{R}=$ $L, n_{D}=1$ ). In this case, $\mathbf{H}_{s d}=h_{s d}$ is scalar (S-D is SISO), $\mathbf{H}_{s r}=\mathbf{h}_{s r}$ is a length $\mathrm{L}$ column vector ( $\mathrm{S}-\mathrm{R}$ is SIMO), and $\mathbf{H}_{r d}=\mathbf{h}_{r d}$ is a length $L$ row vector (R-D is MISO). The information outage probability for the S-D link is the corresponding expression for a SISO link

$$
\operatorname{Pr}\left(C_{s d}<\frac{R}{\alpha}\right)=1-e^{-z}
$$

The information outage probability for the S-R channel is the corresponding expression for a SIMO link

$$
\operatorname{Pr}\left(C_{s r}<\frac{R}{\alpha}\right)=1-e^{-z} \sum_{k=0}^{L-1} \frac{z^{k}}{k !} .
$$

The term $\operatorname{Pr}\left(\alpha C_{s d}+\bar{\alpha} C_{r d}<R\right)$ represents the information outage probability due to the code combining at destination from the two SISO and MISO path. We evaluate this expression as follows:

$$
\begin{aligned}
\operatorname{Pr}\left(\alpha C_{s d}+\bar{\alpha} C_{r d}<R\right) & =\operatorname{Pr}\left[\alpha \log (1+\rho \underbrace{\left|h_{s d}\right|^{2}}_{X})<R\right]+\bar{\alpha} \log (1+\rho \underbrace{\frac{\left\|\mathbf{h}_{r d}\right\|^{2}}{L}}_{Y}) \\
& =\operatorname{Pr}\left[(1+X \rho)^{\alpha}(1+Y \rho)^{\bar{\alpha}}<2^{R}\right] \\
& =\int_{0}^{d} \int_{0}^{c} f_{y}(y) f_{x}(x) d x d y
\end{aligned}
$$

where, $c=\rho^{-1}\left(\frac{2^{\frac{R}{\alpha}}}{(1+y \rho)^{\frac{\alpha}{\alpha}}}-1\right)$ and $d=\left(\frac{2^{\frac{R}{\alpha}}-1}{\rho}\right)$. The expression (3.14) is obtained due to independence of random variables following a similar simplification given in [7]. Here $X, Y$ are independent random variables. $Y$ follows a $\chi^{2}$ distribution with $n_{1}=2 L$ degress of freedom 
(DOF), while $X$ is exponentially distributed with parameter $\lambda=1$ or equivalently $\chi^{2}$ with 2 DOF. The individual pdf's are given by $f_{X}(x)=e^{-x} u(x)$ and $f_{Y}(y)=\frac{L(L y)^{L-1} e^{-L y}}{(L-1) !} u(y)$, where $u(x)$ is a unit step function. Substituting the individual pdfs in (3.14) and integrating over $c, d$ yields

$$
\begin{aligned}
\operatorname{Pr}\left(\alpha C_{s d}+\bar{\alpha} C_{r d}<R\right) & =\int_{0}^{d} \int_{0}^{c} \frac{(L y)^{L-1} e^{-L y}}{(L-1) !} e^{-x} d x d y \\
& =\frac{L^{L}}{(L-1) !} \int_{0}^{d} y^{L-1} e^{-L y}\left[1-e^{-c}\right] d y
\end{aligned}
$$

Substituting (3.12), (3.13), and (3.15) into (3.6), we arrive at the following expression for information outage probability for this case (i.e. $n_{S}=1, n_{R}=L, n_{D}=1$ ) assuming code combining at the destination.

$$
\begin{aligned}
\mathbf{p}_{(1, L, 1)-C C}^{\text {outage }} & =\left[\left(1-e^{-z}\right)\left(1-e^{-z} \sum_{k=0}^{L-1} \frac{z^{k}}{k !}\right)\right. \\
& \left.+\left(e^{-z} \sum_{k=0}^{L-1} \frac{z^{k}}{k !}\right)\left(\frac{L^{L}}{(L-1) !} \int_{0}^{d} y^{L-1} e^{-L y}\left[1-e^{-c}\right] d y\right)\right]
\end{aligned}
$$

\subsubsection{Array at the Destination}

Finally, in this configuration the single antenna relay node assists the single antenna source node and multi-antenna destination node (i.e. $n_{S}=L, n_{R}=1, n_{D}=1$ ). In this case, $\mathbf{H}_{s d}=\mathbf{h}_{s d}$ and $\mathbf{H}_{s r}=\mathbf{h}_{r d}$ are length $L$ row vectors (S-D and R-D are MISO), and $\mathbf{H}_{r d}=h_{s r}$ is scalar ( $\mathrm{S}-\mathrm{R}$ is SISO). The instantaneous capacity of this system is given by (3.4). The information outage occurs when the instantaneous capacity (3.4) is less than the given transmission rate $R$.

$$
\begin{aligned}
\mathbf{p}_{(1,1, L)-C C}^{\text {outage }} & =\operatorname{Pr}\left(C_{D F-C C}<R\right) \\
& =\operatorname{Pr}\left(C_{s r}<\frac{R}{\alpha}\right) \operatorname{Pr}\left(C_{s d}<\frac{R}{\alpha}\right)+\operatorname{Pr}\left(C_{s r}>\frac{R}{\alpha}\right) \operatorname{Pr}\left(\alpha C_{s d}+\bar{\alpha} C_{r d}<R\right)
\end{aligned}
$$


where

$$
\begin{gathered}
C_{s d}=\log \left(1+\rho\left\|\mathbf{h}_{s d}\right\|^{2}\right), \\
C_{r d}=\log \left(1+\rho\left\|\mathbf{h}_{r d}\right\|^{2}\right), \\
C_{s r}=\log \left(1+\rho\left|h_{s r}\right|^{2}\right) .
\end{gathered}
$$

Due to the complexity involved in deriving an analytical expression we present the Monte Carlo simulation results in this case.

\subsubsection{Numerical Results}

In this section we present numerical results for the three terminal relay network with $R=1$ bit per channel use. While the information outage probability when the array is at the destination may be found by performing Monte Carlo integration of (3.17), the expressions for when the array is at the relay and at the source are expressed in terms of an integral. The two expressions, (3.16) and (3.11), can be evaluated using either a numerical integration or a Monte Carlo integration.

Fig. 3.2 presents the outage performance of adaptive DF when one of the three terminals has a two antenna array. For comparison purposes, we also show performance of a direct transmission (essentially $n_{R}=0$ ) with $n_{D}=2,3$ antennas at the destination. Note that all of the considered relaying schemes show diversity (as exhibited by the slope of the curves) that is greater than that of the direct transmission with $n_{D}=2$. When compared against direct transmission with $n_{D}=3$, the relaying schemes have a same slope, but there is a fixed SNR loss. The loss is due to several reasons. First, if the array is at the source or relay, then the power transmitted over each element must be cut in half. Also, there is a loss because there is a chance that the relay decodes incorrectly and is therefore unable to forward the message.

The analytical results also indicate that the most favorable choice for locating the two antennas is at the destination. This can be attributed to the receive diversity gain obtained by both the paths i.e. the S-D path and R-D path. There is SNR loss factor of $L$ in the received signal due to the lack of forward channel knowledge in the S-D, S-R paths or the 


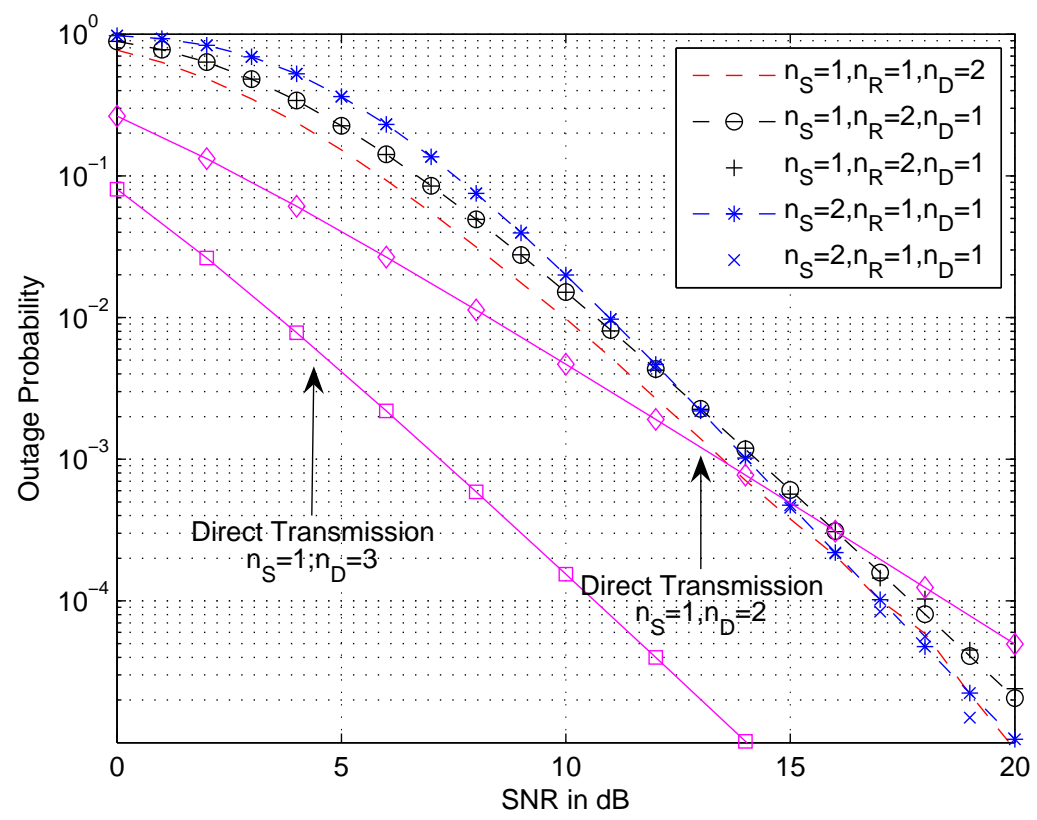

Figure 3.2: Outage probability performance comparison between Monte Carlo integration (symbols) and numerical integration (dashed lines) for the configurations $n_{S}=1, n_{R}=$ $1, n_{D}=2, n_{S}=1, n_{R}=2, n_{D}=1$ and $n_{S}=2, n_{R}=1, n_{D}=1$ with code combining in rayleigh fading environment $(\alpha=0.5)$ with $\mathrm{R}=1$ bit/channel-use.

R-D path in antenna configurations $\left(n_{S}=2, n_{R}=1, n_{D}=1\right)$ and $\left(n_{S}=1, n_{R}=2, n_{D}=1\right)$. Also, the relay retransmits the message only if it can decode correctly. The above reasons lead to the inferior performance when multiple antennas are located at the source or relay. These outage probability results serve as the lower bound on the error rate of an arbitrary code word with the same fixed spectral efficiency.

Fig. 3.3 indicates the impact of increasing the number of receive antennas at the destination in a single relay cooperative network. The asymptotic slope of the curve increases with the increase in the antennas at the destination leading to a considerable performance gain in relay networks. This performance gain may be further exploited by increasing the number of relay nodes.

Figs. 3.4 and 3.5 show the information outage of the relay network with an increasing number of antennas located at the relay and source, respectively. In the low SNR regime, the performance when locating multiple antennas at the relay is better than when locating them at the source. In the configuration $\left(n_{S}=L, n_{R}=1, n_{D}=1\right)$ there is a loss in the received 


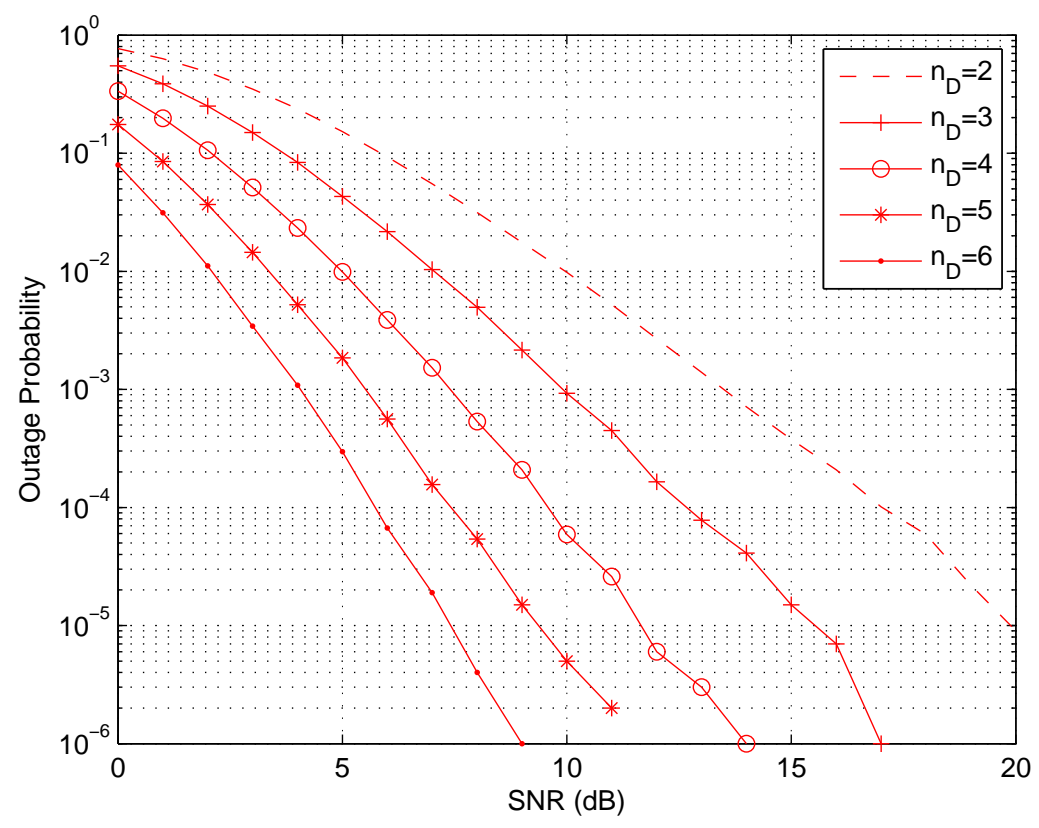

Figure 3.3: Outage probability performance (Monte Carlo integration) with $n_{S}=1 ; n_{R}=1$ and $n_{D}=2,3,4,5,6$ antennas in rayleigh fading environments $(\alpha=0.5)$ with $\mathrm{R}=1$ bit/channel-use.

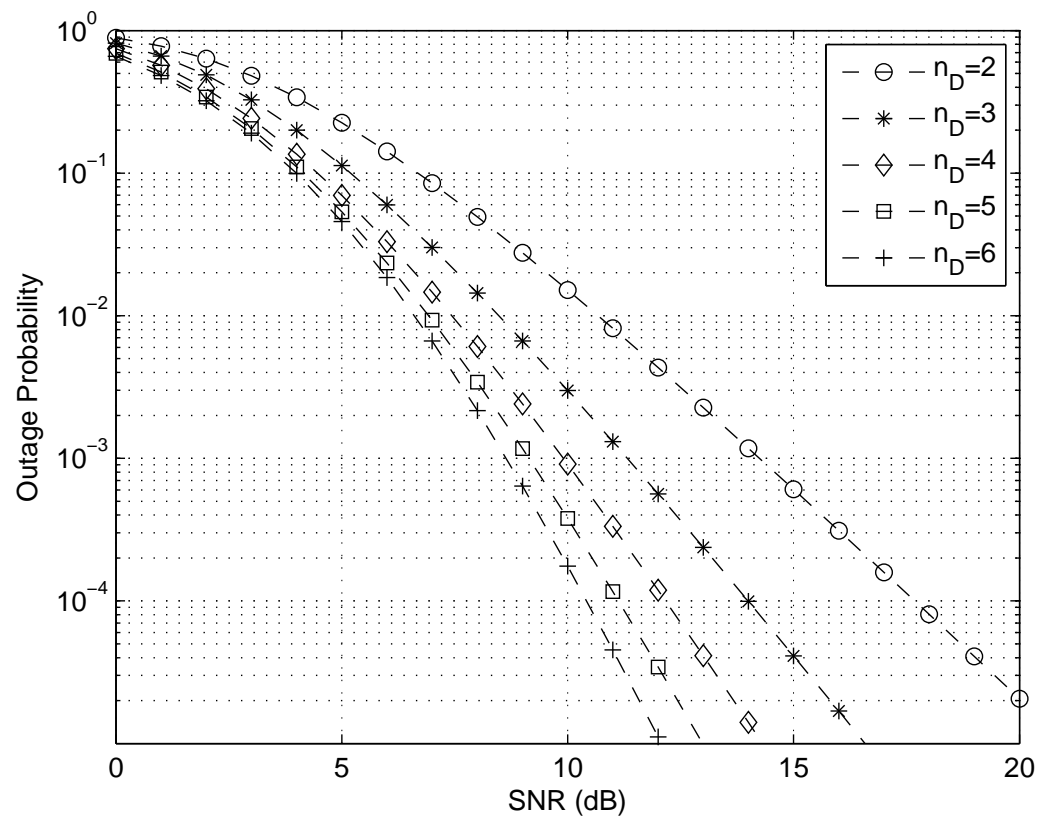

Figure 3.4: Outage probability performance (numerical integration) with $n_{S}=1 ; n_{R}=$ $2,3,4,5,6$ and $n_{D}=1$ antennas for code combining scheme in rayleigh fading environment $(\alpha=0.5)$ with $\mathrm{R}=1 \mathrm{bit} /$ channel-use. 
SNR at the relay due to the the lack of channel knowledge in the S-R path. Also, the relay retransmits only when the message is correct. The above reasons lead to poor performance when compared to $\left(n_{S}=1, n_{R}=L, n_{D}=1\right)$ in the low SNR regime.

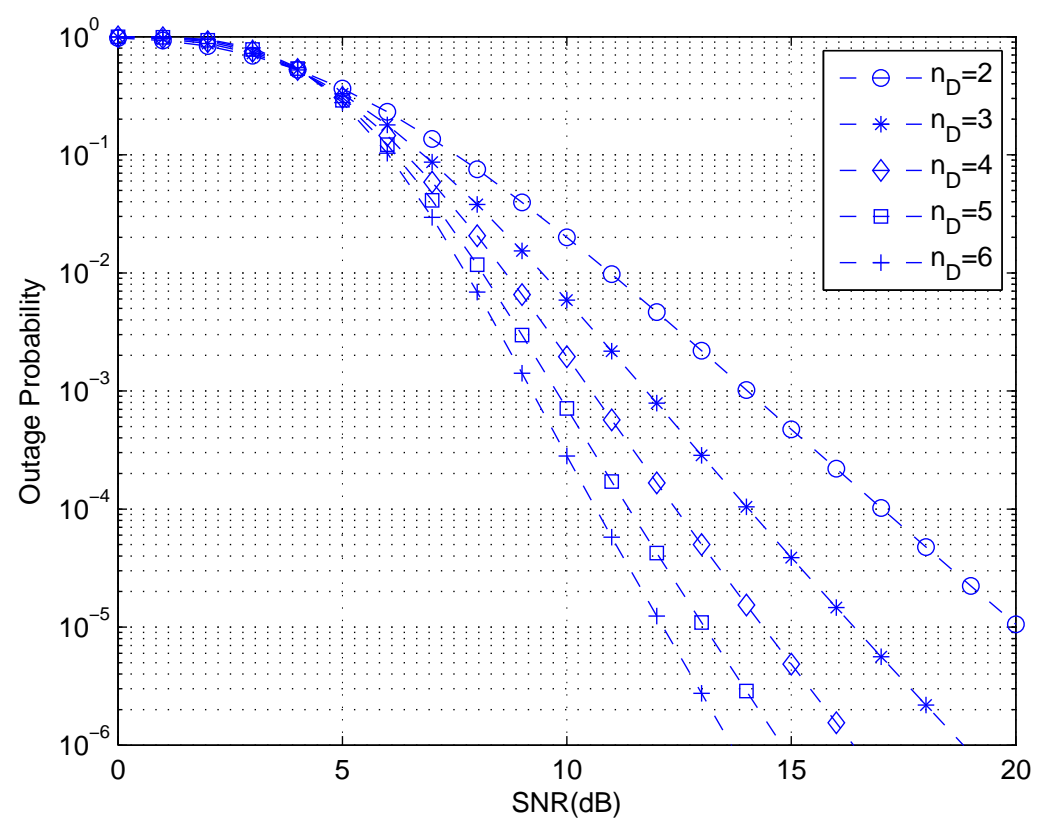

Figure 3.5: Outage probability performance with $n_{S}=2,3,4,5,6 ; n_{R}=1$ and $n_{D}=1$ antennas for code combining scheme in rayleigh fading environment $(\alpha=0.5)$.

The Fig. 3.6 overlays the results shown in Figs. 3.3 through 3.5 to provide a unified view of performance with an array of 2 to 6 elements located at one of the terminals. We observe that in SNR regime, greater than $12 \mathrm{~dB}$, the outage performance obtained by increasing the number of antenna arrays at the relay and source terminals is relatively same. When we locate $L$ antennas at the source, a loss factor of $L$ is involved in the S-R path and the S-D path. Also, the relay forwards the message only if it decodes correctly. In the case of locating multiple antennas at the relay the S-R path is a SIMO channel which accounts for the SNR gain when compared to locating $L$ antennas at the source. Also, the R-D path is a MISO channel, hence a loss factor $L$ is involved whenever the relay decodes the message correctly and forwards it to the destination. Hence in high SNR regime greater than $12 \mathrm{~dB}$ the performance of locating $L$ antennas at the source, relay is the same. It can also be seen that for the same outage and number of antennas, in each antenna configuration, the 


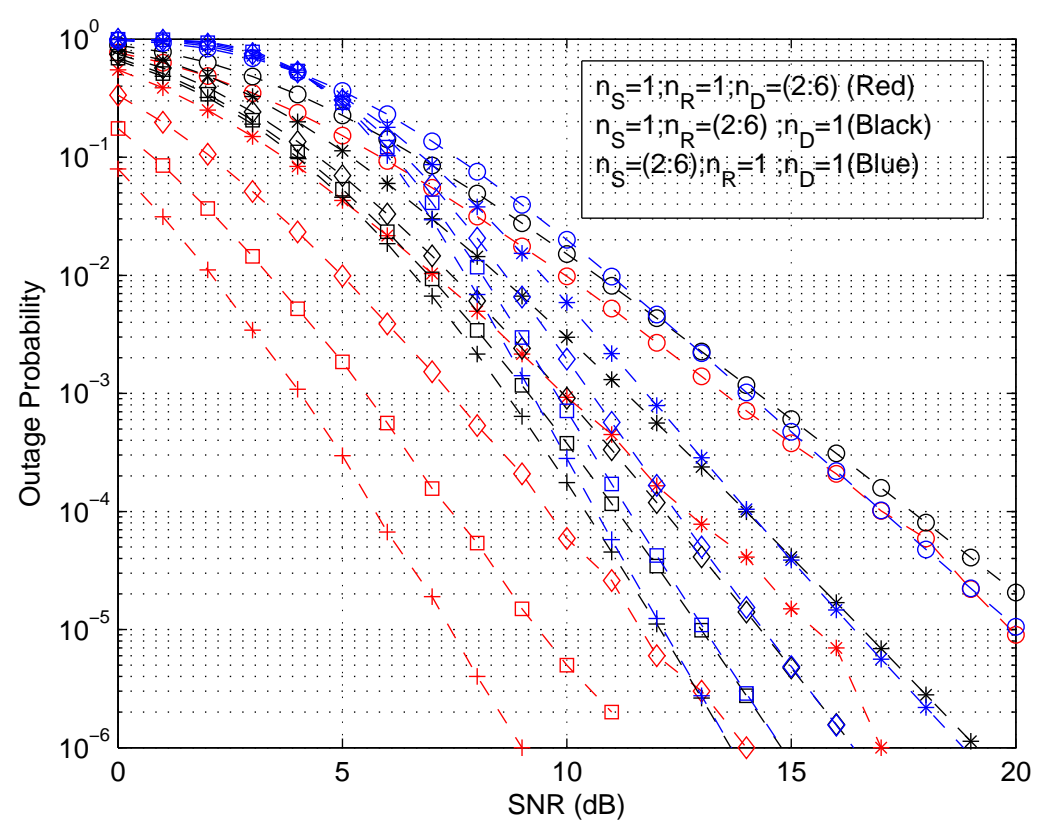

Figure 3.6: Outage probability performance by varying the number of antennas at each node from 2 to 6 in rayleigh fading environment $(\alpha=0.5)$ with $\mathrm{R}=1$ bit/channel-use.

receive diversity has a considerable SNR gain of over $5 \mathrm{~dB}$. The SNR gain is due to the reason that there is no loss factor of $L$ involved in received SNR as the multiple antennas are located at the destination. Hence we conclude that incorporating multiple antennas at the destination yields the best results when there is a requirement to place all the available additional antennas at a single node after allocating one antenna to the source and relay nodes respectively.

\subsection{Relay Network Outage Analysis: Diversity Com- bining}

In this section we discuss some special cases for repetition coding (diversity combining) scheme in the context of incorporating antenna arrays. The instantaneous capacity of the adaptive DF relay network is [24]

$$
C_{D F}=\max \left\{\min \left\{\frac{1}{2} C_{(s d, r d)}, \frac{1}{2} C_{s r}\right\}, \frac{1}{2} C_{s d}\right\}
$$


where $C_{s d}$ is the instantaneous capacity of the source-destination (S-D) path, $C_{s r}$ is the instantaneous capacity of source-relay $(\mathrm{S}-\mathrm{R})$ path, and $C_{(s d, r d)}$ is the instantaneous capacity at destination from the combined signal S-D and relay-destination (R-D) paths. In this case $\alpha=0.5$ since an identical code word is transmitted by the relay if it is able to decode the source message successfully. An outage occurs when the instantaneous capacity is less than the transmission rate $R$ i.e. $C_{D F}<R$. The corresponding information outage probability is

$$
\begin{aligned}
\mathbf{p}_{M R C}^{\text {outage }} & =\operatorname{Pr}\left(C_{D F-M R C}<R\right) \\
& =\operatorname{Pr}\left(C_{s r}<2 R\right) \operatorname{Pr}\left(C_{s d}<2 R\right)+\operatorname{Pr}\left(C_{s r}>2 R\right) \operatorname{Pr}\left(C_{(s d, r d)}<2 R\right)
\end{aligned}
$$

We now evaluate this expression for our three cases of interest, namely the antenna array located at the destination, relay, and source.

\subsubsection{Array at the Destination}

We consider the case that $n_{S}=1, n_{R}=1$, and $n_{D}=L$. In this case, $\mathbf{H}_{s d}=\mathbf{h}_{s d}$ and $\mathbf{H}_{r d}=\mathbf{h}_{r d}$ are length $L$ column vectors, since the S-D and R-D channels are both SIMO, while $\mathbf{H}_{s r}=h_{s r}$ is scalar since the $\mathrm{S}-\mathrm{R}$ channel is single-input, single-output (SISO). The computation of information outage probability $\operatorname{Pr}\left(C_{s d}<2 R\right)$ is identical to that of the direct transmission case given in chapter 2 except that to account for the factor of 2 , we must replace $z_{1}$ given in Chapter 2 with $z=\left(2^{2 R}-1\right) / \rho$. Making this substitution yields. Considering the SIMO S-D link, we get

$$
\operatorname{Pr}\left(C_{s d}<2 R\right)=1-e^{-z} \sum_{k=0}^{L-1} \frac{z^{k}}{k !} .
$$

Considering the SISO S-R link, we get

$$
\begin{aligned}
\operatorname{Pr}\left(C_{s r}<2 R\right) & =\operatorname{Pr}\left[\log \left(1+\rho\left|h_{s r}\right|^{2}\right)<2 R\right] \\
& =\operatorname{Pr}[\left|h_{s r}\right|^{2}<\underbrace{\frac{2^{2 R}-1}{\rho}}_{z}] \\
& =1-e^{-z} .
\end{aligned}
$$

since $\left|h_{s r}\right|^{2}$ follows a exponential distribution. 
The term $\operatorname{Pr}\left(C_{(s d, r d)}<2 R\right)$ represents the information outage probability due to the diversity combining at destination from the two SIMO paths S-D and R-D. The information outage of the diversity combined paths is

$$
\operatorname{Pr}\left(C_{(s d, r d)}<2 R\right)=\operatorname{Pr}\left[\log \left(1+\rho\left(\left\|\mathbf{h}_{s d}\right\|^{2}+\left\|\mathbf{h}_{r d}\right\|^{2}\right)\right)<2 R\right]
$$

In the diversity combining the SNR's add and the instantaneous capacity expression is expressed as single logarithm with sum of SNR's. Hence, the outage probability is (3.25)

$$
\begin{aligned}
& =\operatorname{Pr}[(\underbrace{\left\|\mathbf{h}_{s d}\right\|^{2}}_{X}+\underbrace{\left\|\mathbf{h}_{r d}\right\|^{2}}_{Y})<\underbrace{\frac{2^{2 R}-1}{\rho}}_{z}] \\
& =1-e^{-z} \sum_{k=0}^{2 L-1} \frac{z^{k}}{k !} .
\end{aligned}
$$

Here $X, Y$ are independent $\chi^{2}$ random variables, each with $2 L$ degrees of freedom (DOF), and therefore $X+Y$ is $\chi^{2}$ with $4 L$ DOF. The expression (3.25) is merely the CDF of $X+Y$ $[18]$.

Substituting (3.23), (3.24), and (3.25) into (3.22), we arrive at the following closed form expression for information outage probability for this case (i.e. $n_{S}=1, n_{R}=1, n_{D}=L$ ):

$$
\mathbf{p}_{(1,1, L)-M R C}^{\text {outage }}=\left[\left(1-e^{-z}\right)\left(1-e^{-z} \sum_{k=0}^{L-1} \frac{z^{k}}{k !}\right)+e^{-z}\left(1-e^{-z} \sum_{k=0}^{2 L-1} \frac{z^{k}}{k !}\right)\right] .
$$

When $n_{R}=n_{S}$ and the relay decodes the message successfully, $\mathbf{s}_{r}=\mathbf{s}_{s}$ and the destination maximal ratio (MRC) combines the received source and relay signals in the usual way. On the other hand, if $n_{R} \neq n_{S}$, then $\mathbf{s}_{r}$ cannot equal $\mathbf{s}_{s}$, since the vectors are of differing lengths. In this case, we may assume a two stage encoding process, whereby the message is first encoded with a channel code (defined over an arbitrarily large alphabet) and then modulated using a vector modulator (for instance a space-time code). Both source and relay transmit the same channel codeword, though the modulated symbol vectors will necessarily be different. At the destination, the source and relay signals are individually demodulated, and the demodulator transforms the channel symbol likelihoods into code symbol likelihoods. Since both source and relay transmit the same underlying channel code, the code symbol likelihoods corresponding to the source transmission may be MRC combined with the code 
symbol likelihoods corresponding to the relay transmission, and the aggregate codeword can be passed through the channel decoder.

\subsubsection{Array at the Relay}

Now consider the case that $n_{S}=1, n_{R}=L$, and, $n_{D}=1$. In this case, $\mathbf{H}_{s d}=h_{s d}$ is scalar (S-D is SISO), $\mathbf{H}_{s r}=\mathbf{h}_{s r}$ is a length $L$ column vector (S-R is SIMO), and $\mathbf{H}_{r d}=\mathbf{h}_{r d}$ is a length L row vector (R-D is MISO). The information outage probability for the S-D link is the corresponding expression for a SISO link

$$
\operatorname{Pr}\left(C_{s d}<2 R\right)=1-e^{-z}
$$

The information outage probability for the S-R channel is the corresponding expression for a SIMO link

$$
\operatorname{Pr}\left(C_{s r}<2 R\right)=1-e^{-z} \sum_{k=0}^{L-1} \frac{z^{k}}{k !} .
$$

The information outage probability obtained when diversity combining of the MISO R-D and S-D SISO paths is

$$
\begin{aligned}
\operatorname{Pr}\left(C_{(s d, r d)}<2 R\right) & =\operatorname{Pr}\left[\log \left(1+\rho\left(\frac{\|\left.\mathbf{h}_{r d}\right|^{2}}{L}+\left|h_{s d}\right|^{2}\right)\right)<2 R\right] \\
& =\operatorname{Pr}[(\underbrace{\frac{\|\left.\mathbf{h}_{r d}\right|^{2}}{L}}_{X}+\underbrace{\left|h_{s d}\right|^{2}}_{Y})<\underbrace{\left.\frac{2^{2 R}-1}{\rho}\right]}_{z} \\
& =\frac{L^{L}}{(L-1) !} \int_{0}^{z} x^{L-1} e^{-L x}\left[1-e^{x-z}\right] d x .
\end{aligned}
$$

The derivation of this expression is as follows. Here $X, Y$ are independent random variables. $X$ follows a $\chi^{2}$ distribution with $n_{1}=2 L$ DOF, while $Y$ is exponentially distributed with parameter $\lambda=1$ or equivalently $\chi^{2}$ with 2 DOF. While the CDF of $L X+Y$ is quite simple to find (it is $\chi^{2}$ with $\left.2(L+1) \mathrm{DOF}\right)$, the CDF of $X+Y$ is not straightforward. The CDF of $(X+Y)$ is found using

$$
\begin{aligned}
\operatorname{Pr}(X+Y \leq z) & =\int_{x=0}^{z} \int_{y=0}^{z-x} f_{x y}(x, y) d y d x \\
& =\int_{x=0}^{z} \int_{y=0}^{z-x} f_{x}(x) f_{y}(y) d y d x
\end{aligned}
$$


where the second line comes from the independence of $X$ and $Y$. Substituting the individual pdf's $f_{X}(x)=\frac{L(L x)^{L-1} e^{-L x}}{(L-1) !} u(x)$ and $f_{Y}(y)=e^{-y} u(y)$, where $u(x)$ is the unit step function, yields

$$
\operatorname{Pr}(X+Y<z)=\int_{x=0}^{z} \int_{y=0}^{z-x} \frac{L(L x)^{L-1} e^{-L x}}{(L-1) !} e^{-y} d y d x .
$$

Integrating over y yields (3.29)

Substituting (3.27), (3.28), and (3.29) into (3.22), we arrive at the following expression for information outage probability for this case (i.e. $n_{S}=1, n_{R}=L, n_{D}=1$ )

$$
\begin{gathered}
\mathbf{p}_{(1, L, 1)-M R C}^{\text {outage }}=\left[\left(1-e^{-z}\right)\left(1-e^{-z} \sum_{k=0}^{L-1} \frac{z^{k}}{k !}\right)+\right. \\
\left.\left(e^{-z} \sum_{k=0}^{L-1} \frac{z^{k}}{k !}\right)\left(\frac{L^{L}}{(L-1) !} \int_{0}^{z} x^{L-1} e^{-L x}\left[1-e^{x-z}\right] d x\right)\right] .
\end{gathered}
$$

\subsubsection{Array at the Source}

Finally, consider the case that $\left(n_{S}=L, n_{R}=1, n_{D}=1\right)$. In this case, $\mathbf{H}_{s d}=\mathbf{h}_{s d}$ and $\mathbf{H}_{s r}=\mathbf{h}_{s r}$ are length $L$ row vectors (S-D and S-R are MISO), and $\mathbf{H}_{r d}=h_{r d}$ is scalar (R-D is SISO). The information outage probability for the S-D channel is that of an MISO channel with $L$ transmit antennas

$$
\operatorname{Pr}\left(C_{s d}<2 R\right)=1-e^{-L z} \sum_{k=0}^{L-1} \frac{(L z)^{k}}{k !} .
$$

The information outage probability for the S-R channel is also that of an MISO channel with $L$ transmit antennas

$$
\operatorname{Pr}\left(C_{s r}<2 R\right)=1-e^{-L z} \sum_{k=0}^{L-1} \frac{(L z)^{k}}{k !} .
$$

The information outage probability obtained when diversity combining of the MISO S-D 
and SISO R-D paths is

$$
\begin{aligned}
\operatorname{Pr}\left(C_{(s d, r d)}<2 R\right) & =\operatorname{Pr}\left[\log \left(1+\rho\left(\frac{\left\|\mathbf{h}_{s d}\right\|^{2}}{L}+\left|h_{r d}\right|^{2}\right)\right)<2 R\right] \\
& =\operatorname{Pr}[(\underbrace{\frac{\|\left.\mathbf{h}_{s d}\right|^{2}}{L}}_{X}+\underbrace{\left|h_{r d}\right|^{2}}_{Y})<\underbrace{\left.\frac{2^{2 R}-1}{\rho}\right]}_{z} \\
& =\frac{L^{L}}{(L-1) !} \int_{0}^{z} x^{L-1} e^{-L x}\left[1-e^{x-z}\right] d x .
\end{aligned}
$$

The derivation is identical to that for (3.29).

Substituting (3.31), (3.32), and (3.33) into (3.22), we arrive at the following expression for information outage probability for this case (i.e. $n_{S}=L, n_{R}=1, n_{D}=1$ )

$$
\begin{aligned}
\mathbf{p}_{(L, 1,1)-M R C}^{\text {outage }}= & {\left[\left(1-e^{-L z} \sum_{k=0}^{L-1} \frac{(L z)^{k}}{k !}\right)^{2}+\left(e^{-L z} \sum_{k=0}^{L-1} \frac{(L z)^{k}}{k !}\right)\right.} \\
& \left.\left(\frac{L^{L}}{(L-1) !} \int_{0}^{z} x^{L-1} e^{-L x}\left[1-e^{x-z}\right] d x\right)\right]
\end{aligned}
$$

\subsubsection{Numerical Results}

In this section we present numerical results for the three terminal relay network assuming the diversity combining with $R=1$ bit per channel use. We obtain the information outage probability of for case when the relay repeats the same code word transmitted by the source, by evaluating the expression (3.26),(3.30),(3.34) using either a numerical integration. Fig. 3.7 indicates the impact of increasing the number of receive antennas at the destination in a single relay cooperative network. The asymptotic slope of the curve increases with an increase in the number of antennas. Also, an SNR gain can be observed with each additional antenna at the destination. Hence we conclude that incorporating multiple antennas at the destination yields the best results when there is a requirement to place all the available additional antennas at a single node, after allocating one antenna initially to source and relay node, which is the same conclusion as that of the code combining scheme. Also, a comparison of code-combining and diversity combining scheme is discussed in 3.5, which indicates the sub-optimality of $\alpha=0.5$. 


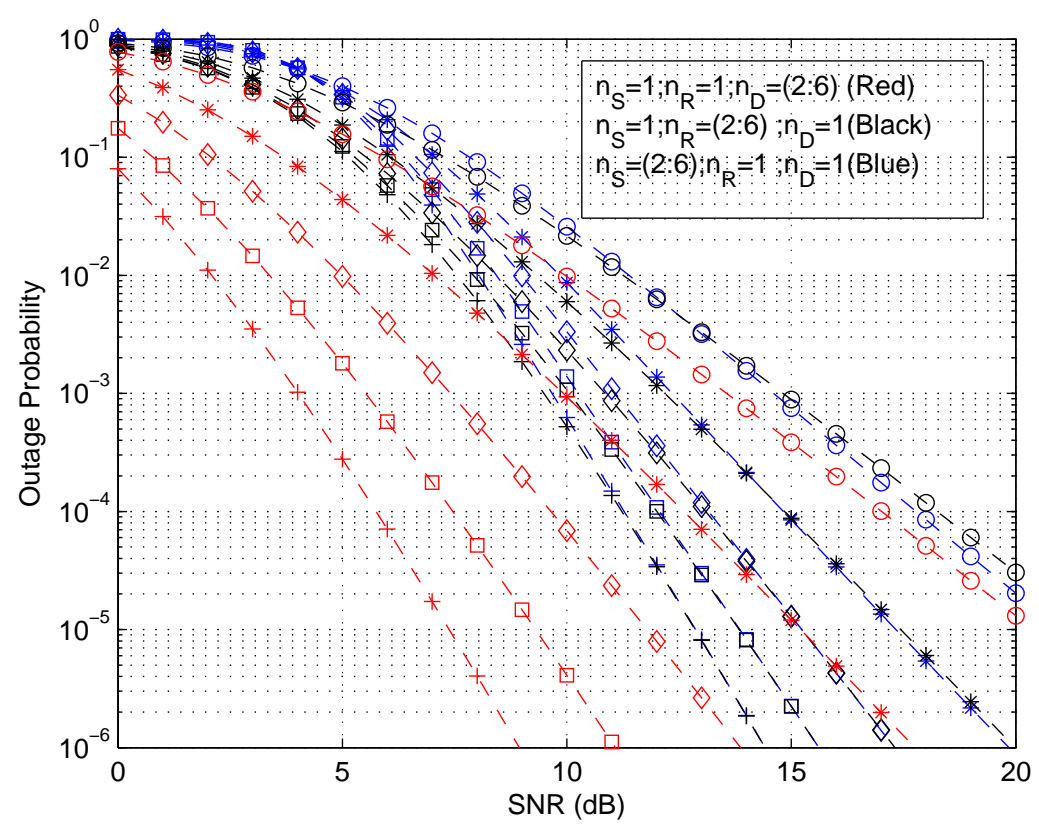

Figure 3.7: Outage probability performance of diversity combining (numerical integration) by varying the number of antennas at each node from 2 to 6 with $\mathrm{R}=1$ bit/channel-use.

\subsection{Relay Network Outage Analysis: Arrays at multi- ple nodes}

In the following sections we present the information outage probability performance of code combining scheme and some special cases of diversity combining when $n_{S}=n_{R}$ by locating the antenna arrays at multiple nodes simultaneously. The performance results for both the combining schemes obtained by Monte Carlo simulation indicate the advantages of decode-and-forward relays with multiple antennas.

\subsubsection{Code Combining}

We present the performance of locating antenna arrays at multiple nodes simultaneously assuming code combining at the destination. The instantaneous capacity for such networks 
is given by (3.4). The information outage expression is formulated as

$$
\begin{aligned}
\mathbf{p}_{\left(n_{S}, n_{R}, n_{D}\right)}^{\text {outage }} & =\operatorname{Pr}\left(C_{D F-C C}<R\right) \\
& =\operatorname{Pr}\left(C_{s r}<\frac{R}{\alpha}\right) \operatorname{Pr}\left(C_{s d}<\frac{R}{\alpha}\right) \\
& +\operatorname{Pr}\left(C_{s r}>\frac{R}{\alpha}\right) \operatorname{Pr}\left(\alpha C_{s d}+\bar{\alpha} C_{r d}<R\right)
\end{aligned}
$$

where,

$$
\begin{gathered}
C_{s r}=\log \operatorname{det}\left(I_{n_{R}}+\frac{\rho}{n_{S}} \mathbf{H}_{s r} \mathbf{H}_{s r}^{H}\right) \\
C_{s d}=\log \operatorname{det}\left(I_{n_{D}}+\frac{\rho}{n_{S}} \mathbf{H}_{s d} \mathbf{H}_{s d}^{H}\right) \\
C_{r d}=\log \operatorname{det}\left(I_{n_{D}}+\frac{\rho}{n_{R}} \mathbf{H}_{r d} \mathbf{H}_{r d}^{H}\right)
\end{gathered}
$$

The equations (3.38),(3.37),(3.38) represent the instantaneous MIMO capacities of the respective point-point paths.

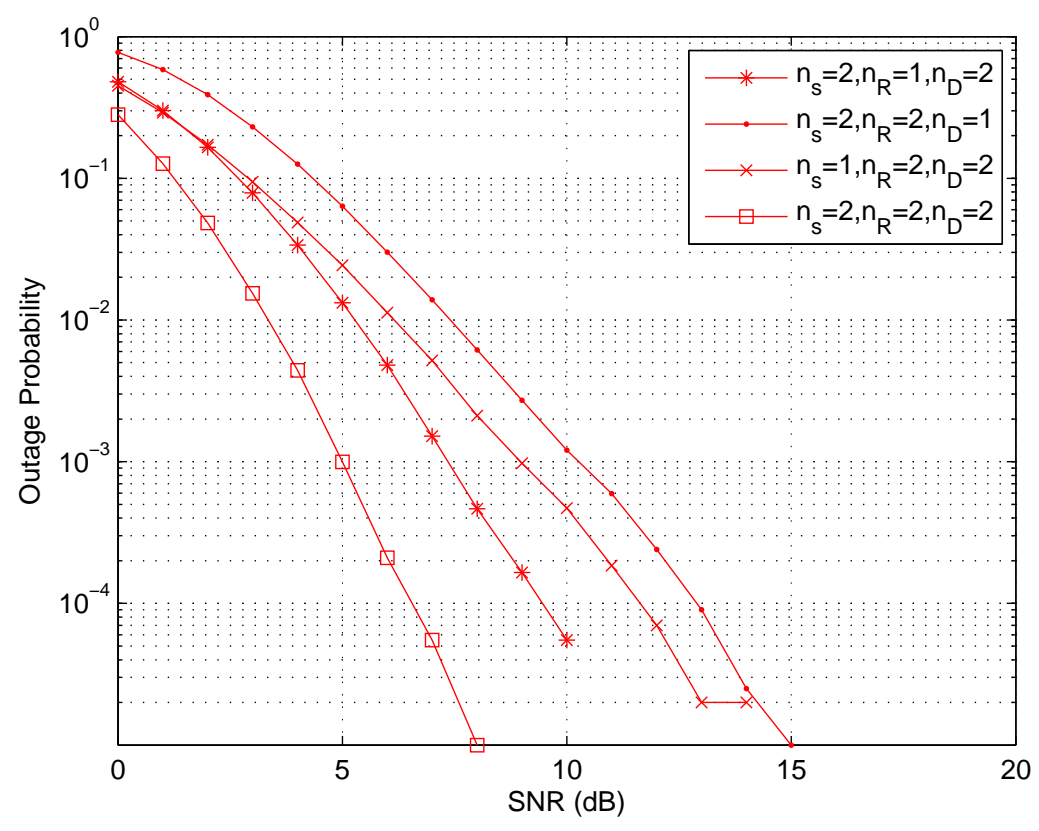

Figure 3.8: Outage probability performance of code combining scheme obtained via Monte Carlo simulation by incorporating antenna arrays at multiple nodes simultaneously with $\mathrm{R}=1 \mathrm{bit} /$ channel-use. $(\alpha=0.6)$

Fig. 3.8 presents the results obtained by Monte Carlo evaluation of (3.35) for the following antenna configurations $\left(n_{T}=1, n_{R}=2, n_{D}=2\right),\left(n_{T}=2, n_{R}=2, n_{D}=1\right),\left(n_{T}=2, n_{R}=\right.$ 
$\left.1, n_{D}=2\right)$ with $\alpha=0.5$. In the antenna configuration $\left(n_{T}=2, n_{R}=2, n_{D}=1\right)$ the S-D, RD paths are a MISO links and S-R path is a MIMO channel. There is a loss factor of present in the outage expression of (MISO paths) as the transmitting node lacks the forward channel knowledge. The S-R path, a MIMO channel, will retransmit only if it can decode perfectly. The above reasons lead to a worse performance when compared to the other configurations.

In the antenna configuration $\left(n_{T}=1, n_{R}=2, n_{D}=2\right)$ the S-R, S-D paths are SIMO channels, R-D path is a MIMO channel. This configuration we can see that there is no requirement of channel knowledge at any transmitting node. Although, the R-D is MIMO channel the relay participates in cooperation only if it is able to decode the information correctly, which leads to the lack of performance.

The $\left(n_{T}=2, n_{R}=1, n_{D}=2\right)$ configuration has a MISO channel in the S-R path, a MIMO channel in the S-D and the SIMO channel in R-D paths. This results in the best outage performance when compared with the performance of $\left(n_{T}=2, n_{R}=2, n_{D}=\right.$ $1),\left(n_{T}=1, n_{R}=2, n_{D}=2\right)$. Even though there is loss factor involved in the S-R path due to lack of the forward channel knowledge, this configuration leads to better results than the above schemes. This is due to reason the relay incorporates only single antenna and the R-D path is a SIMO path which involves no loss in the received SNR.

Hence, we conclude that if 2 additional antennas are available then the best choice is to place one each at the source and destination nodes in a cooperative relay network. From the antenna configuration considered for analysis, if additional antennas greater than 2 are available then the first choice is to distribute the additional antennas equally among all the nodes in three terminal relay network. If equal distribution is not possible then the antennas should be distributed such that the number of antennas at the relay is always less than both the source and destination antennas. These conclusions are further supported in section 3.6.

\subsubsection{Diversity Combining}

Here, we present the information outage probability allowing the multiple antennas to be located at more than terminal in a cooperative relay network assuming diversity combining at the destination. The instantaneous capacity for the diversity combining scheme is given 
by (3.21). The information outage expression is

$$
\begin{aligned}
\mathbf{p}_{\left(n_{S}, n_{R}, n_{D}\right)}^{\text {outage }} & =\operatorname{Pr}\left(C_{D F-D C}<R\right) \\
& =\operatorname{Pr}\left(C_{s r}<2 R\right) \operatorname{Pr}\left(C_{s d}<2 R\right)+\operatorname{Pr}\left(C_{s r}>2 R\right) \operatorname{Pr}\left(C_{(s d, r d)}<2 R\right)
\end{aligned}
$$

where,

$$
\begin{array}{r}
C_{s r}=\log \operatorname{det}\left(I_{n_{R}}+\frac{\rho}{n_{S}} \mathbf{H}_{s r} \mathbf{H}_{s r}^{H}\right) \\
C_{s d}=\log \operatorname{det}\left(I_{n_{D}}+\frac{\rho}{n_{S}} \mathbf{H}_{s d} \mathbf{H}_{s d}^{H}\right) \\
C_{(s d, r d)}=\log \operatorname{det}\left(I_{n_{D}}+\frac{\rho}{n_{S}}\left[\mathbf{H}_{s d} \mathbf{H}_{s d}^{H}\right]+\frac{\rho}{n_{R}}\left[\mathbf{H}_{r d} \mathbf{H}_{r d}^{H}\right]\right)
\end{array}
$$

The expression (3.42) is the instantaneous capacity obtained due to the diversity combining scheme at the destination [11]. We now evaluate the the expression (3.39) for special case, i.e., equal number of antennas at source, relay, and different number of antennas at the destination using Monte Carlo integration with spectral efficiency $R=1$ bit per channel use.

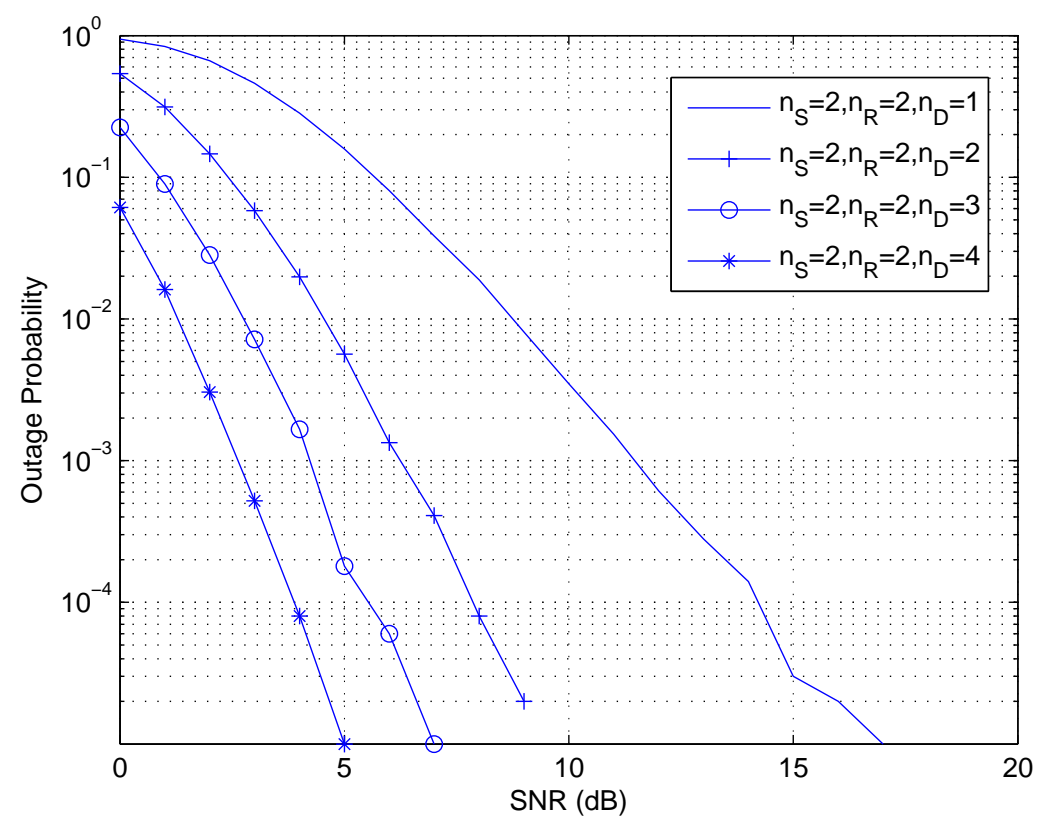

Figure 3.9: Outage probability performance of diversity combining scheme obtained via Monte Carlo integration by incorporating multiple antennas in rayleigh fading environment with $\mathrm{R}=1 \mathrm{bit} /$ channel-use. 
The Fig. 3.9 represents the diversity gain obtained by incorporating multiple antenna relay network. With each additional receiver antenna we obtain additional diversity gain. These result indicate the advantages of diversity combining using multi antenna terminals in a relay network.

\subsection{Code Combining Vs Diversity Combining}

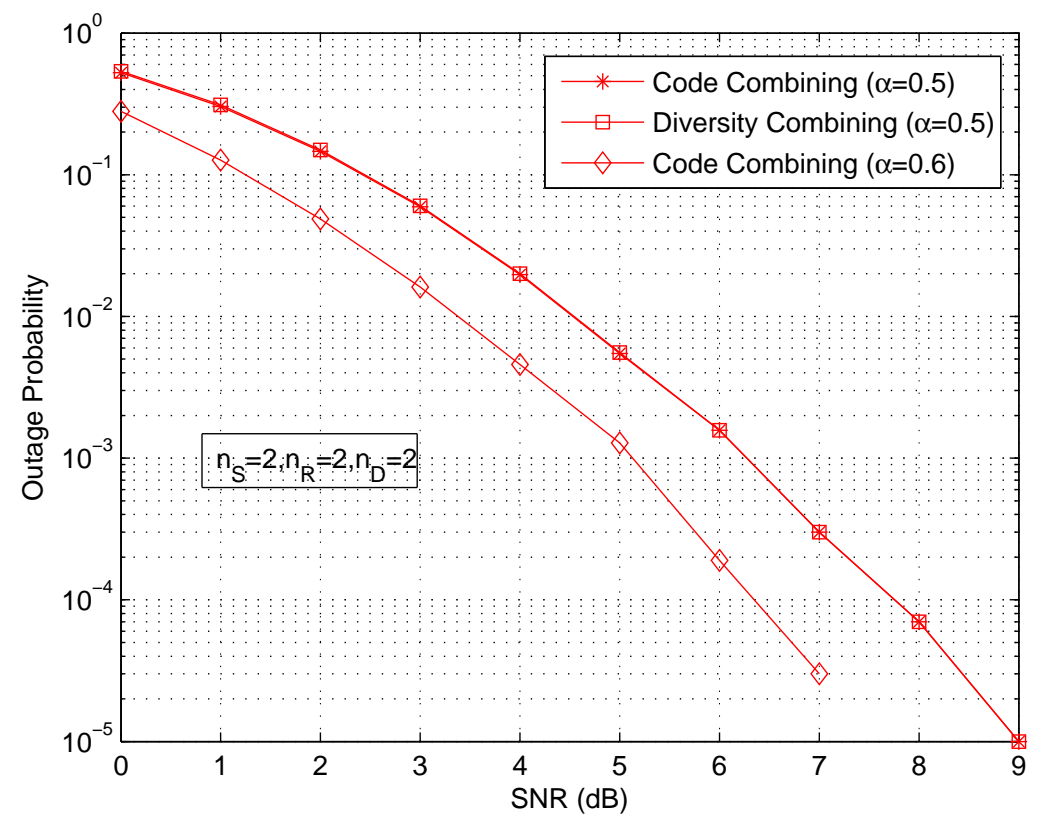

Figure 3.10: Comparison of code combining and diversity combining scheme using Monte Carlo integration with $\mathrm{R}=1$ bit/channel-use.

In Fig. 3.10 we compare the information outage probability for the code combining with $\alpha=0.5$ and diversity combining scheme, for the similar antenna configuration. We observe that the code combining scheme provides no additional gain which indicates that the value $\alpha=0.5$ is suboptimal. When $\alpha=0.6$ there is an additional $1 \mathrm{~dB}$ SNR gain. The performance with $\alpha=0.6$ in code combining scheme highlights the advantages of code combining. 


\subsection{Cooperation Rate}

In previous section, for the case of code combining, we fix the optimal fraction of symbols that are being transmitted from the source i.e. $\alpha=0.5$ leading to a fixed rate cooperation, which indicated that this value is suboptimal. Hence, in order to achieve the additional coding gain due to code combining for a given antenna configuration, we identify a fixed $\alpha$ by simulating the outage performance against $\alpha$ at a fixed SNR.

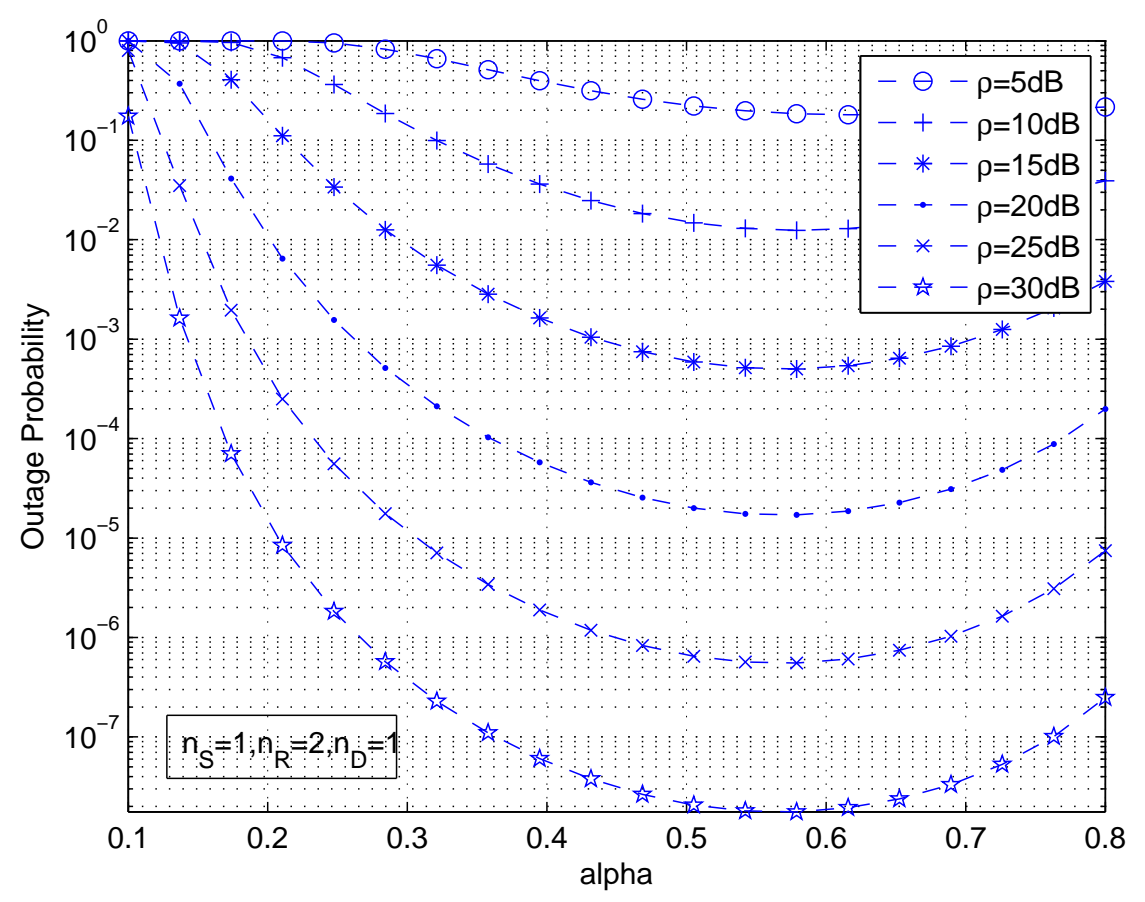

Figure 3.11: Outage probability performance of code combining scheme obtained by numerical integration with $\left(n_{S}=1, n_{R}=2, n_{D}=1\right)$ at various $\mathrm{SNR}$ with $\mathrm{R}=1$ bit/channel-use.

The Fig. 3.11 represents the outage performance of a fixed antenna configuration at different SNR values. We observe that the $\alpha$ remains constant for different values of SNR.

In Fig. 3.12 we identify the optimal $\alpha$ by plotting the outage performance of the code combining scheme for the first case using, where we assume that all the additional antennas are located at a particular node for a fixed SNR. For the antenna configurations, $\left(n_{T}=\right.$ $\left.L, n_{R}=1, n_{D}=1\right),\left(n_{T}=1, n_{R}=L, n_{D}=1\right)$ we numerically integrate the one dimensional integrals (3.16), (3.11), and for $\left(n_{T}=1, n_{R}=1, n_{D}=L\right)$ we present the results using Monte Carlo integration of (3.17) with $R=1$ bit per channel use. Also, the Montecarlo integration 


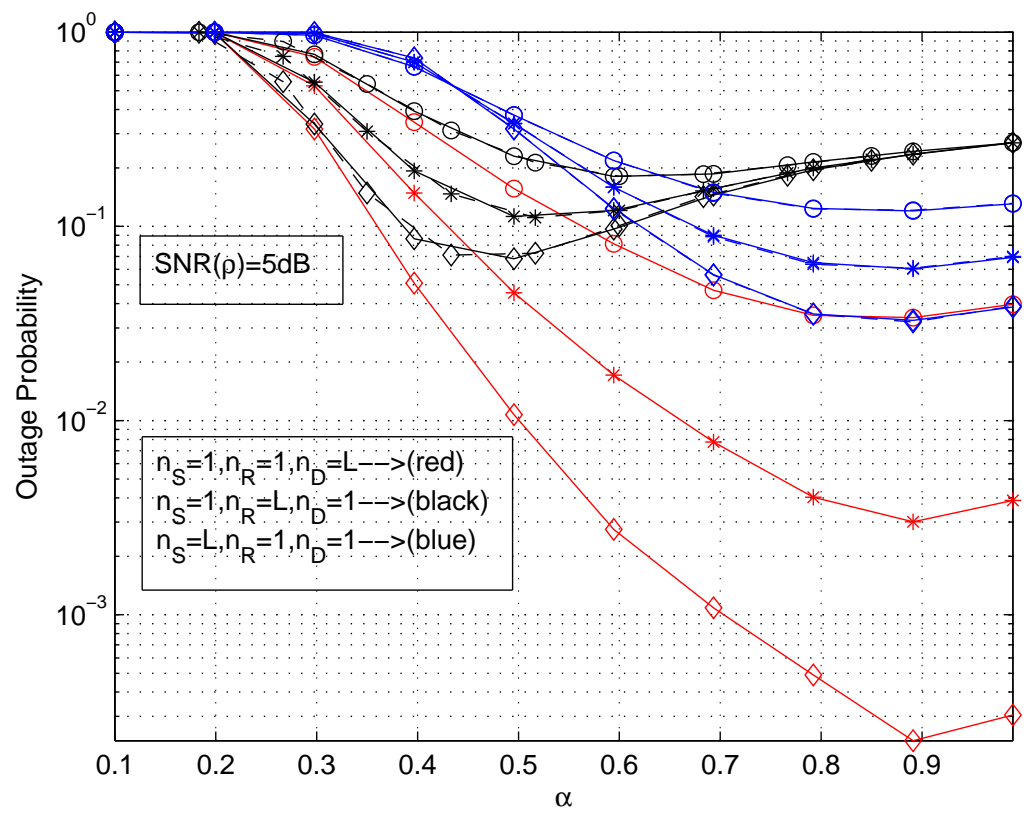

Figure 3.12: Outage probability performance of code combining scheme obtained by Monte Carlo integration (solid lines) and numerical integration (dashed lines) for different antennas $(L=2,3,4)$ at a fixed SNR of $5 \mathrm{~dB}$ with $\mathrm{R}=1 \mathrm{bit} /$ channel-use.

of $\left(n_{T}=L, n_{R}=1, n_{D}=1\right),\left(n_{T}=1, n_{R}=L, n_{D}=1\right)$ is presented for comparison purposes. For the antenna configuration $\left(n_{T}=L, n_{R}=1, n_{D}=1\right)$ we observe that allowing the source to transmit a larger fraction of symbols yields better results because of the multiple antenna location at source terminal. In the configuration $\left(n_{T}=1, n_{R}=L, n_{D}=1\right)$ the source transmits a lower fraction of the symbols as it is equipped with single antenna and hence allows the relay to transmit a larger fraction which is equipped with $L$ antennas. With increasing the number of additional antennas at the relay we can observe that the value of $\alpha$, i.e., the optimal value $\alpha$ for the source transmission decreases. Also, we can observe that by increasing the fraction of code symbols from the source the performance of the system converges to that of the point-to-point scheme. For the antenna configuration $\left(n_{T}=1, n_{R}=\right.$ $\left.1, n_{D}=L\right)$, it is always beneficial for the source to transmit a larger fraction of the codeword than the relay when we increase antennas number of antennas at the destination.

The Fig. 3.13 represent the outage performance of different antenna configurations at various $\alpha$ using a Monte Carlo simulation for the second case, i.e, we allow the antenna array to be located at more than one terminal simultaneously in the relay network. The results 


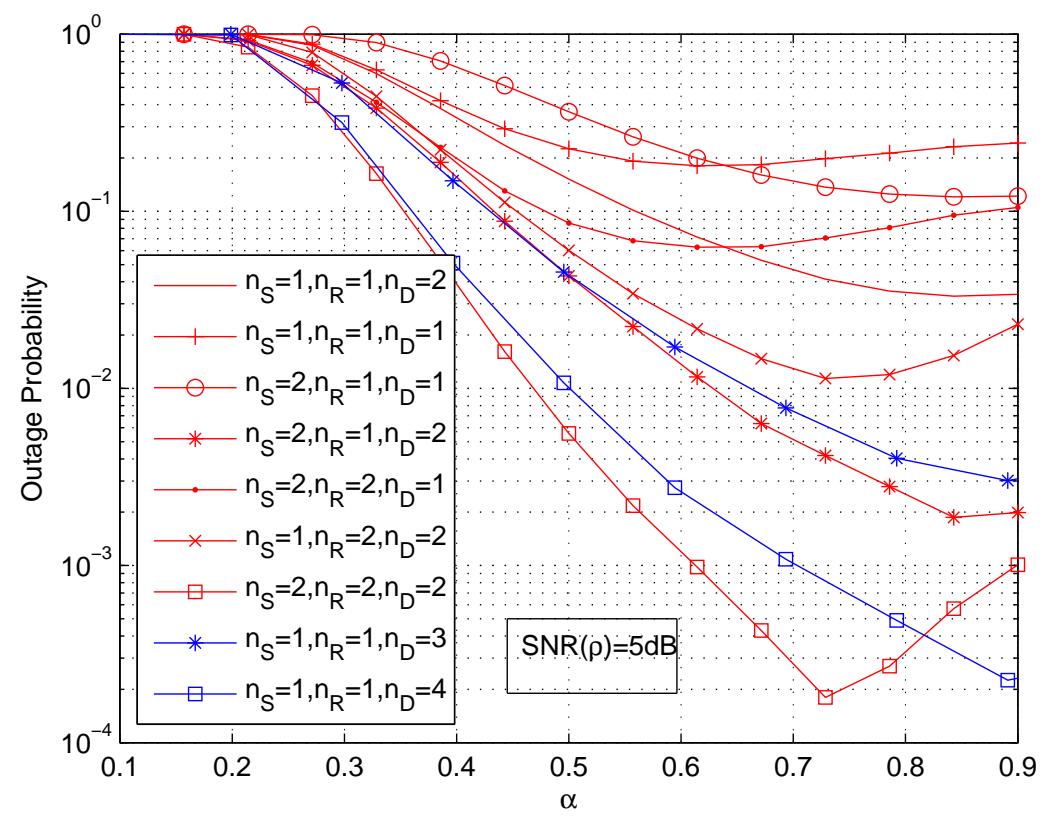

Figure 3.13: Outage probability performance of code combining scheme obtained via Monte Carlo integration by incorporating multiple antennas in rayleigh fading environment with $\mathrm{R}=1 \mathrm{bit} /$ channel-use.

indicate that the optimal value $\alpha$, the fraction of code symbol transmission from the source varies for each antenna configuration. In the antenna configuration $\left(n_{T}=2, n_{R}=2, n_{D}=1\right)$ when we increase the value of $\alpha$ beyond 0.6 the performance converges to that of the pointto-point scheme with $\left(n_{S}=2, n_{D}=1\right)$. For $\left(n_{T}=1, n_{R}=2, n_{D}=2\right)$ we see that the outage performance converges to the point-to-point scheme with beyond $\alpha=0.73$. For the configuration $\left(n_{T}=2, n_{R}=1, n_{D}=2\right)$ we can observe that its advantageous for source to transmit a larger fraction of symbols and beyond $\alpha=0.85$ the performance converges to point-to-point system. Finally, with $\left(n_{T}=2, n_{R}=2, n_{D}=2\right)$ antenna configuration we can observe that the performance converges to a MIMO system beyond $\alpha=0.72$. In essence results convey that we can take full advantage of coded cooperation at particular value of cooperation rate i.e. $\alpha$ and when the source is allowed to transmit beyond this $\alpha$ performance converges to the point-to-point scheme. We present the results for $\left(n_{T}=1, n_{R}=1, n_{D}=3\right)$ and $\left(n_{T}=1, n_{R}=1, n_{D}=4\right)$. When we compare the above configurations against the $\left(n_{T}=2, n_{R}=1, n_{D}=2\right)$ (i.e with same number of antennas), results support our conclusions arrived in section 3.4.1 that the best choice is to accommodate minimum number of antennas 
at the relay.

\subsection{Chapter Summary}

This chapter presents an information-theoretic overview of cooperative relay channels with multiple antenna array terminals. Results presented in terms of information outage probability for various antenna configurations in rayleigh fading channels indicate the best possible distribution of antennas in a relay networks for two different cases of code and diversity combining schemes. In particular, the performance of different antenna configurations under code combining for both the cases, obtained by varying the cooperation rate i.e. the fraction of code symbols $\alpha$ highlights the value at which coded cooperation is beneficial in a relay network. 


\section{Chapter 4}

\section{Practical Cooperative Space-Time}

\section{Architectures}

In the previous chapter we perform an information-theoretic analysis and study the impact of locating multiple antennas in a three terminal relay network in terms of information outage probability. The outage probability results provide an insight into the achievable diversity gains in a relay network equipped with multiple antennas at various nodes. The information theoretic results form a motivating factor for system design using practical schemes. Sendonaris et.al. were the first to realize the advantages of user cooperative coding in [3]. Zhao and Valenti in [25] also propose distributed turbo code for the relay channels. Both the proposed schemes assume single antennas at the all the terminals of the cooperative wireless network. In [9] Dohler et.al. propose a practical virtual antenna array with two transmit antennas at the base station with amplify-and-forward (AF) relaying schemes employing space-time block codes (STBC) [13] with an application in cellular networks. In [9] Dohler et.al propose the virtual antenna array network (VAA), where the base station (source) uses two antennas to send the 2-by-2 Alamouti space-time code to a pair of handsets (relay and destination). Each handset is equipped with one antenna, but is able to mimic a two antenna receiver by exchanging information via the amplitude-and-forward (AF) protocol. In [10] Herhold et.al. provides a unifying analysis of AF protocols using distributed STBC. The above works consider an AF protocol which is associated with the drawback of relaying the amplified version of noise present on the source-relay channel [5]. 
In this chapter we propose practical cooperative space time architecture with repetition coding at the relay, i.e., we perform diversity combining at the destination. Also, in order to over come the this limitation of AF relaying we explore the diversity and throughput gains in a MIMO relay system using an adaptive DF relaying in rayleigh fading environment. First, we exploit the diversity gains by simulating a cooperative relay transmission scheme assuming adaptive decode-and-forward (DF) repetition of the orthogonal space-time code word proposed by Alamouti in [13]. Second, we simulate the performance of cooperative VBLAST [1] assuming a repetition DF at the relay. Results are presented in terms of BER and normalized throughput, giving us an insight into the achievable gains by incorporating multiple antennas in a simple adaptive DF cooperative relay network.

\subsection{Cooperative Space-Time Transmission Architectures}

We consider the system model as described in Chapter 3 for the simulation of transmission with multiple antennas at all terminals using orthogonal space-time block codes (STBC) and vertical-BLAST (VBLAST).

\subsubsection{Cooperative Transmission Using STBC}

In this scheme during the first phase the source broadcasts an Alamouti space-time code word. The relay and destination listen to the source. The relay decodes the information using a maximal ratio combining as specified in [13]. During the second phase, if the relay decodes the message correctly (verified via a CRC code, for example), it retransmits the message to the destination. Since the two channels i.e. the source-destination (S-D) and relay-destination (R-D) paths are separable we perform independent combining for each path and then the resulting estimates are summed to yield the final decision statistic. This process is a MIMO version of MRC which is given as follows.

$$
\mathbf{y}=\mathbf{H}_{s d}^{H} \mathbf{y}_{s d}+\mathbf{H}_{r d}^{H} \mathbf{y}_{r d}
$$


with decisions provided by

$$
\begin{aligned}
& \tilde{\mathbf{s}}[1]=\operatorname{sign}\{\Re[\mathbf{y}[1]]\}+\mathrm{j}[\operatorname{sign}\{\Im[\mathbf{y}[1]]\}] \\
& \tilde{\mathbf{s}}[2]=\operatorname{sign}\{\Re[\mathbf{y}[2]]\}+\mathrm{j}[\operatorname{sign}\{\Im[\mathbf{y}[2]]\}]
\end{aligned}
$$

As the maximum-likelihood (ML) estimates are the same for ML estimation in white noise, no equalization is required. If the $\mathrm{CRC}$ check is incorrect then the destination performs the decision with the estimates obtained from the first phase of transmission.

\subsubsection{Cooperative VBLAST Transmission}

In the cooperative VBLAST transmission scheme, during the first phase the source transmits independent messages over two antennas. To create a signal suitable for suboptimal detection the relay first forms a vector statistic

$$
\begin{aligned}
\mathbf{z} & =\mathbf{H}_{s r}^{H} \mathbf{y}_{s r} \\
& =\mathbf{H}_{s r}^{H} \mathbf{H}_{s r} \mathbf{s}+\mathbf{H}_{s r}^{H} \mathbf{n}_{r} \\
& =\boldsymbol{\Omega} \mathbf{s}+\boldsymbol{\omega}
\end{aligned}
$$

where $\boldsymbol{\Omega} \triangleq \mathbf{H}_{s r}^{H} \mathbf{H}_{s r}$ and $\boldsymbol{\omega} \triangleq \mathbf{H}_{s r}^{H} \mathbf{n}_{r} \sim \mathcal{N}_{c}\left(\mathbf{0}, N_{o} \boldsymbol{\Omega}\right)$. This process is essentially the MIMO version of MRC. We whiten the statistic forming,

$$
\begin{aligned}
\mathbf{u} & =\boldsymbol{\Omega}^{-\frac{1}{2}} \mathbf{Z} \\
& =\boldsymbol{\Omega}^{\frac{1}{2}} \mathbf{S}+\tilde{\mathbf{n}}_{r}
\end{aligned}
$$

where $\tilde{\mathbf{n}}_{r} \sim \mathcal{N}_{c}\left(\mathbf{0}, N_{o} \mathbf{I}_{n_{R}}\right)$. The VBLAST detection scheme using successive interference cancelation technique as specified in [1] is performed to decode the received message.

During the second phase if the decoded message is correct (verified via a CRC code, for example) the relay forwards the information to the destination. At the receiver, since the paths S-D and R-D are separable, we perform independent combining on both channels. For S-D path,

$$
\begin{aligned}
\mathbf{z}_{1} & =\mathbf{H}_{s d}^{H} \mathbf{y}_{s d} \\
& =\mathbf{H}_{s d}^{H} \mathbf{H}_{s d} \mathbf{s}+\mathbf{H}_{s d}^{H} \mathbf{n}_{d_{1}} \\
& =\boldsymbol{\Omega}_{1} \mathbf{s}+\boldsymbol{\omega}_{1}
\end{aligned}
$$


where $\boldsymbol{\Omega}_{1} \triangleq \mathbf{H}_{s d}^{H} \mathbf{H}_{s d}$ and $\boldsymbol{\omega}_{1} \triangleq \mathbf{H}_{s d}^{H} \mathbf{n}_{d_{1}} \sim \mathcal{N}_{c}\left(\mathbf{0}, N_{o} \boldsymbol{\Omega}_{1}\right)$. We whiten the statistic forming,

$$
\begin{aligned}
\mathbf{u}_{1} & =\boldsymbol{\Omega}_{1}^{-\frac{1}{2}} \mathbf{z}_{1} \\
& =\Omega_{1}^{\frac{1}{2}} \mathbf{S}+\tilde{\mathbf{n}}_{d_{1}}
\end{aligned}
$$

where $\tilde{\mathbf{n}}_{d_{1}} \sim \mathcal{N}_{c}\left(\mathbf{0}, N_{o} \mathbf{I}_{n_{D}}\right)$. Next we perform the similar operation in R-D path,

$$
\begin{aligned}
\mathbf{z}_{2} & =\mathbf{H}_{r d}^{H} \mathbf{y}_{r d} \\
& =\mathbf{H}_{r d}^{H} \mathbf{H}_{r d} \mathbf{s}+\mathbf{H}_{r d}^{H} \mathbf{n}_{d_{2}} \\
& =\boldsymbol{\Omega}_{2} \mathbf{s}+\boldsymbol{\omega}_{2}
\end{aligned}
$$

where $\boldsymbol{\Omega}_{2} \triangleq \mathbf{H}_{r d}^{H} \mathbf{H}_{r d}$ and $\boldsymbol{\omega}_{2} \triangleq \mathbf{H}_{r d}^{H} \mathbf{n}_{d_{2}} \sim \mathcal{N}_{c}\left(\mathbf{0}, N_{o} \boldsymbol{\Omega}_{2}\right)$. We whiten the statistic forming,

$$
\begin{aligned}
\mathbf{u}_{2} & =\boldsymbol{\Omega}_{2}^{-\frac{1}{2}} \mathbf{z}_{2} \\
& =\Omega_{2}^{\frac{1}{2}} \mathbf{S}+\tilde{\mathbf{n}}_{d_{2}}
\end{aligned}
$$

where $\tilde{\mathbf{n}}_{d_{2}} \sim \mathcal{N}_{c}\left(\mathbf{0}, N_{o} \mathbf{I}_{n_{D}}\right)$. The estimates from both paths after two phases of transmission are summed up to yield the statistic $\tilde{\mathbf{u}}=\mathbf{u}_{1}+\mathbf{u}_{2}$. $\tilde{\mathbf{u}}$ is of the form $\mathbf{y}=\mathbf{H s}+\mathbf{n}$ which allows to perform the VBLAST ZF, MMSE detection as mentioned in [26] using successive interference cancelation (SIC) method.

The end-to-end bit error rate (BER) of the cooperative system after the two transmission phases is given by $B E R_{\text {end-end }}=\operatorname{BER}_{1 \mathrm{D}}\left(1-\mathrm{P}_{\mathrm{R}}\right)+\mathrm{P}_{\mathrm{R}} B \mathrm{R}_{2 \mathrm{D}}$, where $\mathrm{P}_{\mathrm{R}}$ is the probability of relaying, $\mathrm{BER}_{1 \mathrm{D}}$ is the $\mathrm{BER}$ at destination when the relay does not forward the message and $\mathrm{BER}_{2 \mathrm{D}}$ is the BER at destination when the relay forwards the information.

\subsection{Simulation Results}

For all simulations, we assume that the channel between each node has the same average SNR. At each node, the normalized transmit power of each bit across all the transmit antennas during each phase is 1 . We assume perfect channel knowledge at all the receiving nodes. Fig. 4.1 illustrates the BER performance of cooperative DF relay transmission using space time block code. The $(2: 2)$ alamouti STBC scheme along with the maximal ratio combining 


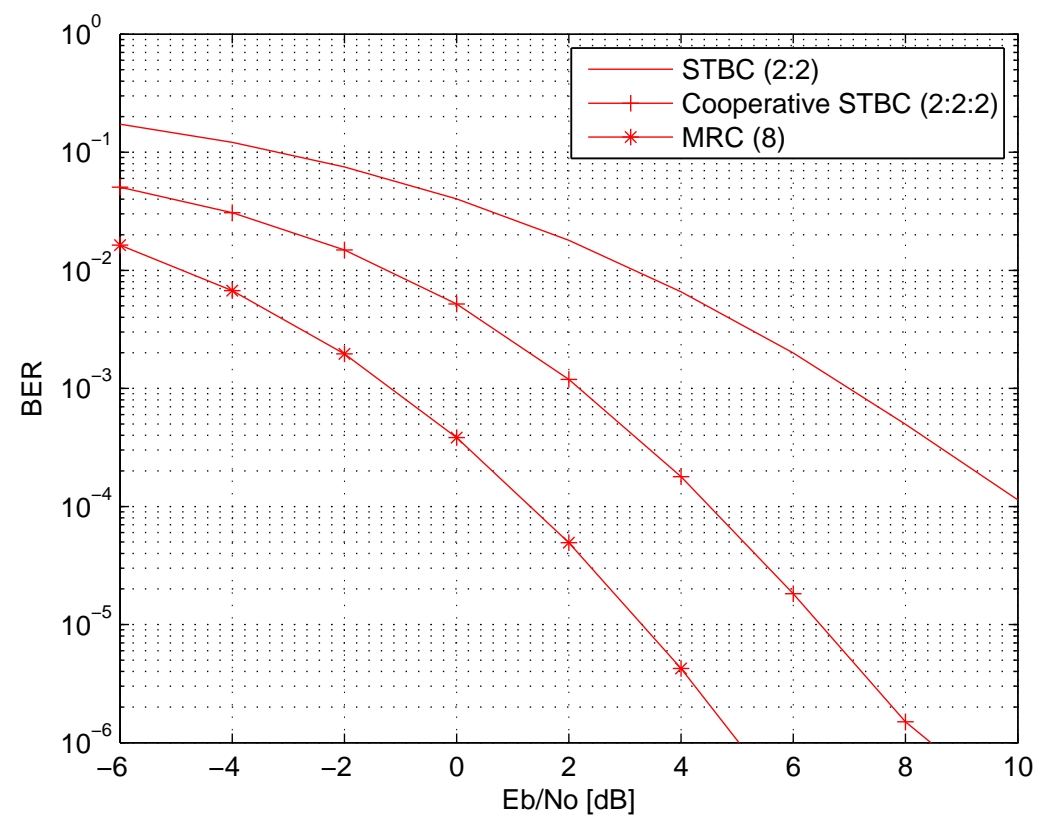

Figure 4.1: BER and diversity performance of cooperative DF STBC. The BER curve of 2:2 STBC is plotted for comparison, as is the performance of maximum ratio combining with 8 antennas with $\mathrm{R}=1$ bit/channel-use

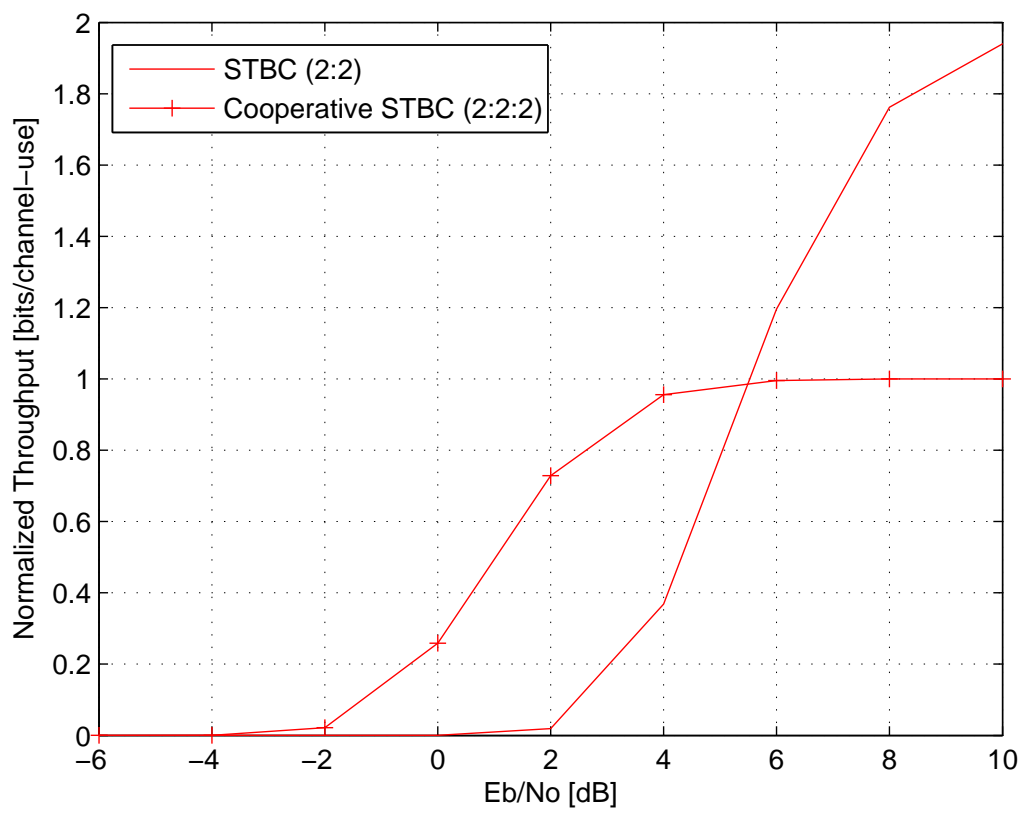

Figure 4.2: Throughput performance of cooperative DF STBC. The Throughput of 2:2 STBC is plotted for comparison 
scheme is presented for comparison purposes. The cooperative DF multi-antenna relay networks achieves a higher diversity when compared to a (2:2) alamouti STBC transmission. A comparison with BER performance of MRC with 8 antennas indicates that the cooperative space time transmission achieves full diversity. There is an SNR loss of 3dB due to splitting of power across antennas at each terminal.

Fig. 4.2 indicates the normalized throughput performance of the cooperative DF relay transmission using space-time block codes, along with (2:2) alamouti transmission scheme. Normalized throughput, $T$, is calculated as

$$
T=R\left(1-\mathrm{BER}_{\text {end-end }}\right)^{M} \mathrm{bits} / \text { channel-use, }
$$

For transmission using cooperative DF STBC $T=T_{s t b c}\left(0 \leq T_{s t b c} \leq 1\right)$ is the normalized throughput, $R=R_{s t b c}$ is the normalized rate and $M=256$ is the packet size. The proposed cooperative DF STBC transmission operates at rate $R_{s t b c}=1$. This transmission scheme results in a better throughput performance in the low SNR regime when compared to $(2: 2)$ Alamouti transmission scheme.

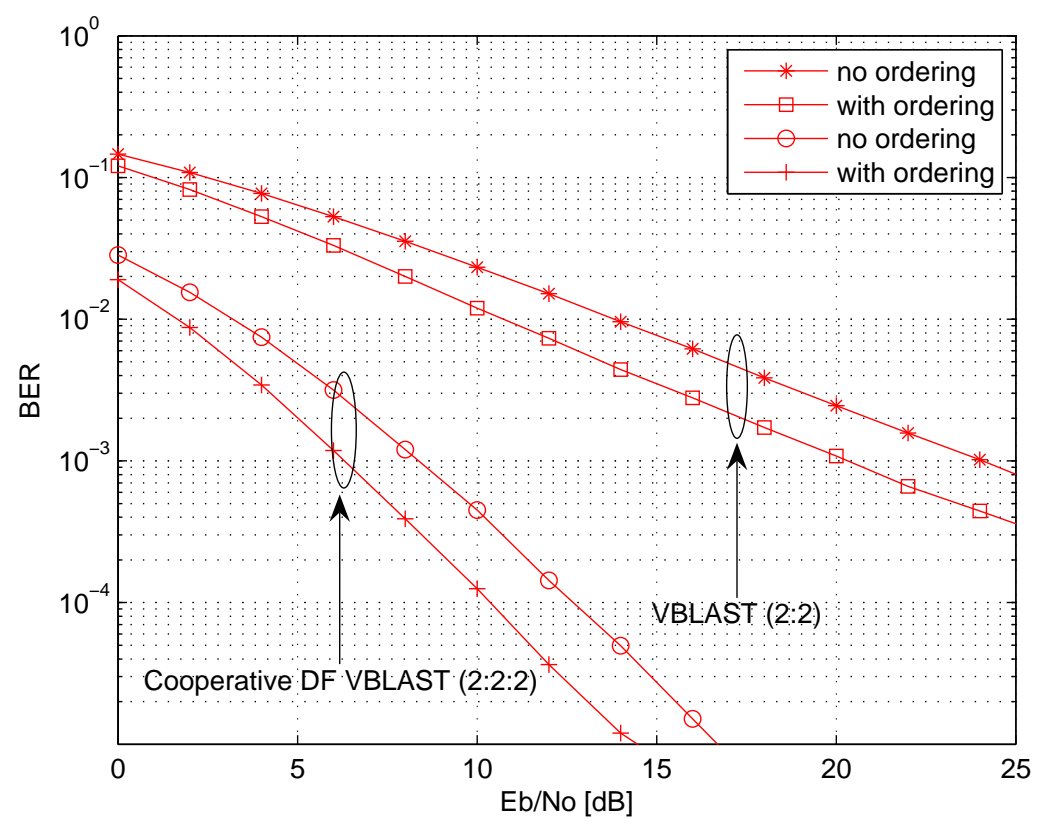

Figure 4.3: BER performance of cooperative DF VBLAST using ZF with and without ordered SIC with $\mathrm{R}=2$ bits/channel-use. The BER curve of 2:2 VBLAST for the same detection scheme is plotted for comparison 


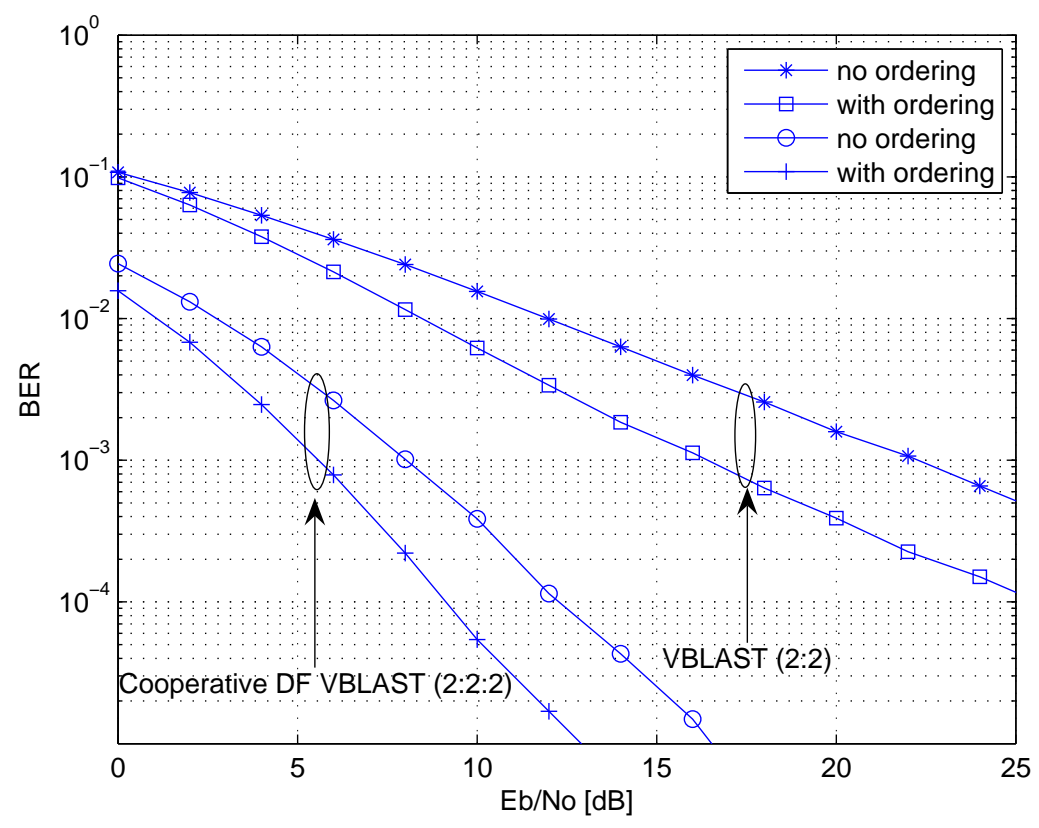

Figure 4.4: BER performance of cooperative DF VBLAST using MMSE with and without ordered SIC with $\mathrm{R}=2$ bits/channel-use. The BER curve of 2:2 VBLAST for the same detection scheme is plotted for comparison.

In Fig. 4.3, Fig. 4.4 we present the BER performance results of cooperative DF VBLAST zero-forcing (ZF), minimum mean square error (MMSE) using SIC with ordered detection and without ordering. The (2:2) VBLAST ZF, MMSE is also presented for comparison purposes. Results indicate that there is increase in diversity gain due to the presence of cooperative DF MIMO relay in wireless network.

For the cooperative DF relay transmission using VBLAST the normalized throughput is $T=T_{\text {vblast }}\left(0 \leq T_{\text {vblast }} \leq 2\right)$, the normalized rate $R=R_{\text {vblast }}$ and $M=256$ is the packet size. The proposed cooperative DF VBLAST transmission operates at rate $R_{v b l a s t}=2$. Fig. 4.5 is the normalized throughput result for the cooperative VBLAST DF relay transmission using ZF with and without ordered SIC. Also the (2:2) VBLAST using the same detection scheme is shown for comparison purposes. We can see that in the mid-SNR regime the cooperative VBLAST using ZF with ordered and without ordered SIC exhibits a better performance when compared to (2:2) VBLAST detection for the same SIC detection. Fig. 4.6 shows the normalized throughput curves of the SIC MMSE detection using a cooperative DF VBLAST scheme, along with a (2:2) VBLAST MMSE detection for comparison. These results indicate 


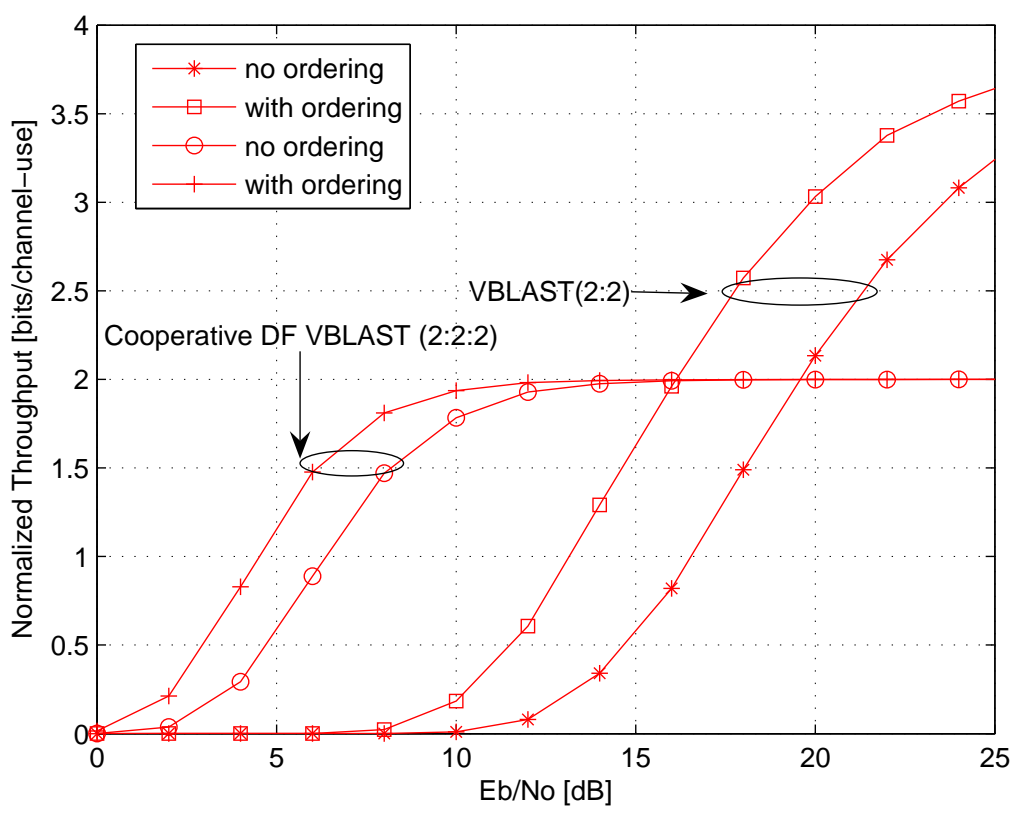

Figure 4.5: Throughput performance of cooperative DF VBLAST using ZF, with and without ordered SIC. The BER curve of 2:2 VBLAST for the same detection scheme is plotted for comparison

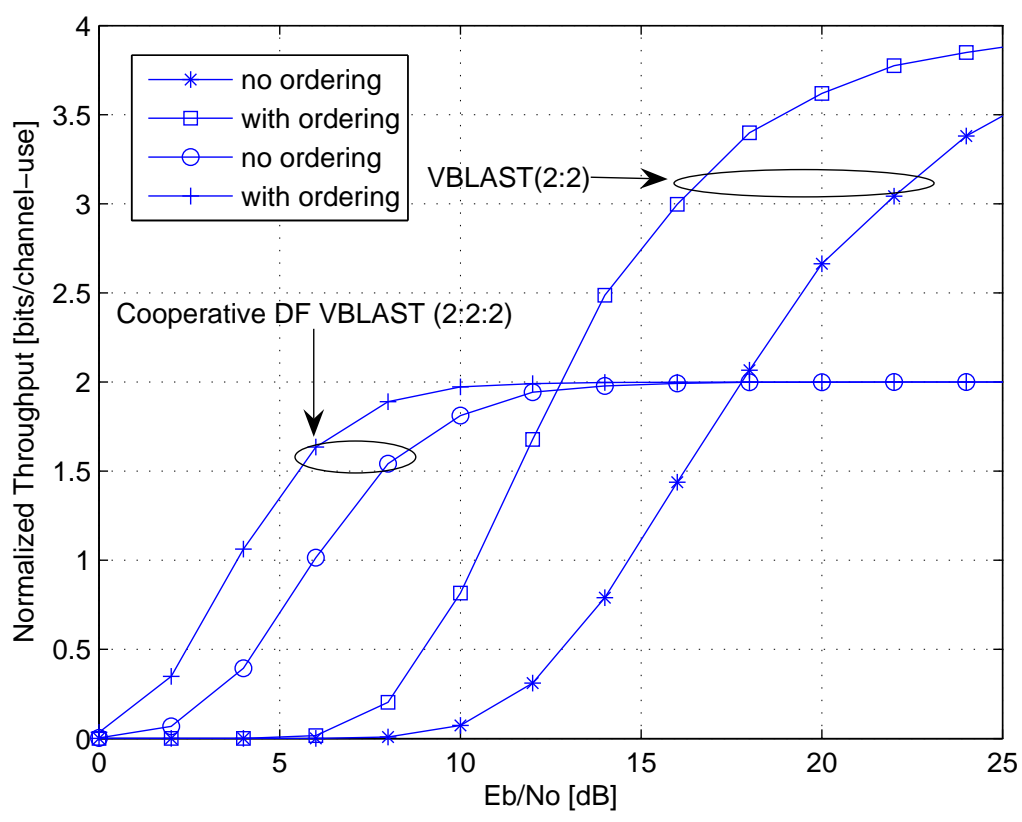

Figure 4.6: Throughput performance of cooperative DF VBLAST using MMSE, with and without ordered SIC. The BER curve of 2:2 VBLAST for the same detection scheme is plotted for comparison 
a better performance in the mid-SNR regime by the cooperative system when compared to the respective $(2: 2)$ VBLAST.

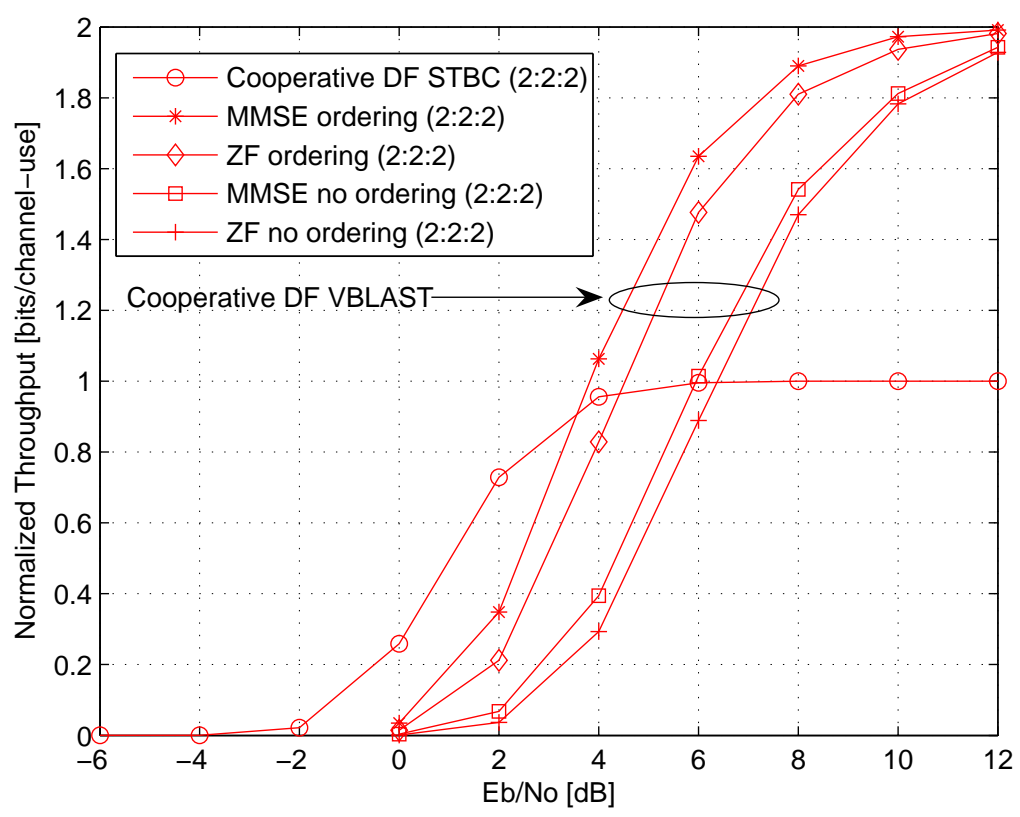

Figure 4.7: Throughput comparison of cooperative STBC and ZF,MMSE VBLAST with and without ordered SIC.

Fig. 4.7 is a unifying comparison of throughput curves obtained using cooperative DF relay for both STBC and VBLAST transmissions. We can see that in the low SNR regime the cooperative DF STBC provides a better throughput when compared to the cooperative DF VBLAST transmission with a rate penalty. The cooperative VBLAST operates at twice the rate when compared to cooperative DF STBC in mid-SNR, high SNR regime.

\subsection{Chapter Summary}

In this chapter we design and simulate a cooperative relay network assuming a practical code structure with multiple antennas at all nodes. The adaptive DF protocol at the relay provides a full diversity and high throughput when compared to the conventional pointpoint MIMO system. Also, we observe that cooperative communication through multiple antennas provide an increased spatial diversity gain also better throughput gains in low SNR, mid-SNR regime for STBC, VBLAST transmissions. 


\section{Chapter 5}

\section{Conclusions}

\subsection{Summary of Results and Conclusion}

In Chapter 2 we initially describe the MIMO channel and characterizes the performance of various antenna array architectures in terms of outage capacity and outage probability for quasi-static flat fading environments. Additionally, we examine the performance of practical space-time codes such as the transmit diversity scheme [13] and VBLAST architectures [26].

In Chapter 3 we describe the simple three terminal relay network employing multiple antennas at all nodes of a wireless network. Based on the closed form expressions obtained for information outage probability in Chapter 2, first, we derive the information outage probability expressions by constraining all the additional antennas to source, relay or destination for coded cooperation (code combining) and repetition coding (diversity combining).

In case of code combining we present the results by numerically integrating the expressions derived when the array is located at the source or relay. When the array is located at the destination we present the results obtained by Monte-Carlo integration. For the diversity combining case, when the array is at the destination a closed form expression obtained is evaluated and when the array is located at the source or relay we present the outage performance results by numerically integrating the one dimensional integrals. Second, we simulate the outage performance of a code combining scheme by allowing multiple nodes to accommodate antenna arrays simultaneously. We simulate the outage performance of the diversity combining scheme for a few special cases of interests in the second case. In addi- 
tion, we compare the code combining with diversity combining scheme at $\alpha=0.5$. Also, we simulate the outage performance at different the cooperation rates i.e fraction of codesymbol transmission from the source for both the cases assuming code cooperation, at a particular SNR.

From the results presented in this chapter we have the following conclusions. In the first case, where we have the constraint to locate all the antennas at a particular node, the best choice is to locate all the additionally available antennas at the destination after allocating one antenna to the source and the relay node. In the second case, for code combining scheme if 2 additional antennas are available, from the results obtained for the antenna configuration considered, we conclude that the best choice is to place one each at the source and destination nodes in a cooperative relay network. If additional antennas greater than 2 are available then first choice is to distribute equally among all the nodes in three terminal relay network. If equal distribution is not possible then the antennas should be distributed such that the number of antennas at the relay is always less than both the source and destination antennas. Also, we conclude that the code combining is suboptimal at $\alpha=0.5$ and identify the optimal cooperation rate for the source and relay for both cases.

In Chapter 4 we present the simulation results assuming practical code structures among a three terminal multi-antenna relay network using transmit diversity scheme [13] and spatial multiplexing architectures, VBLAST [1]. In case of cooperative STBC we conclude that the adaptive DF protocol at the relay provides a full diversity and high throughput in low SNR regime when compared to the conventional point-point MIMO system. Also, we conclude that the cooperative VBLAST operates at twice the rate when compared to cooperative DF STBC in mid-SNR, high SNR regime.

\section{$5.2 \quad$ Future Work}

This section describes the possible extensions of research presented in this thesis.

1. In this thesis we analyze the outage performance of a simple three terminal relay network, with multiple antenna nodes, employing a decode-and-forward relay node. This analysis assume gaussian inputs. An interesting extension is to perform similar 
analysis under modulation constraints.

2. We assume that channel is known to all the receiving nodes and the transmitting node lacks the forward channel knowledge. This analysis can be extended assuming the channel is known to the transmitter.

3. We propose a cooperative transmit diversity scheme and VBLAST architectures. Here, we assume a repetition coding at the relay (diversity combining). A possible extension is to perform simulation incorporating code combining at the destination i.e. the relay retransmits a different part of the codeword (incremental redundancy).

4. We assume a simple three terminal relay network and perform our analysis for different antenna array configurations. Extension of this analysis using antenna arrays to a network of nodes similar to [24] would lead to optimizing the resources of wireless network. 


\section{References}

[1] G. J. Foschini and M. J.Gans, "On limits of wireless communications in a fading environment when using multiple antennas," Wireless Personal Commun., vol. 6, no. 3, pp. 311-355, March 1998.

[2] David Tse and Pramod Viswanath, Fundamentals of Wireless Communication, 1st ed., New York, NY: Cambridge University Press, 2005.

[3] A. Sendonaris, E. Erkip, and B. Aazhang, "User cooperation diversity-part I and part II," IEEE Trans. Commun., vol. 51, no. 11, pp. 1927-1948, Nov. 2003.

[4] A. Nosratinia, T.E. Hunter, and A. Hedayat, "Cooperative communication in wireless networks," IEEE Commun. Magazine, vol. 42, no. 10, pp. 74-80, Oct. 2004.

[5] J. N. Laneman, David N. C. Tse, and G. W. Wornell, "Cooperative diversity in wireless networks:efficient protocols and outage behaviour," IEEE Trans. Inform. Theory, vol. 50, no. 12, pp. 3062-3080, Dec. 2004.

[6] Aggelos Anastasio Bletsas, Intelligent Antenna Sharing in Cooperative Diversity Wireless Networks, Ph.D. thesis, Massachusetts Institute of Technology, Cambridge, MA, September. 2005.

[7] T. Hunter, S. Sanayei, and A. Nosratinia, "Outage analysis of coded cooperation," IEEE Trans. Inform. Theory, vol. 52, no. 2, pp. 375-391, Feb. 2006.

[8] J. N. Laneman and G. W. Wornell, "Distributed space-time coded protocols for exploiting cooperative diversity in wireless networks," IEEE Trans. Inform. Theory, vol. 49, no. 10, pp. 2415-2525, Oct. 2003.

[9] M. Dohler, E. Lefranc, and H. Aghvami, "Space time block codes for virtual antenna arrays," in Proc. IEEE Personal Indoor and Mobile Radio Commun. Conf, Lisbon, Portugal, Sep. 2002.

[10] P. Herhold, E. Zimmermann, and G. Fettweis, "On the performance of cooperative amplify-and-forward relay networks," in 5th International ITG Conference on Source and Channel Coding (SCC), Erlangen, Germany, Jan. 2004.

[11] D. P. Palomar, A. Agustin, O. Muñoz, and J. Vidal, "Decode-and-forward protocol for cooperative diversity in multi-antenna wireless networks," in Proc. CISS, Princeton, NJ, Mar. 2004. 
[12] Bin Zhao, Multiterminal Relay Networks: Performance Bounds, Protocol Design and Channel Coding Strategies, Ph.D. thesis, West Virginia University, Morgantown, WV, April. 2004.

[13] Sivash M. Alamouti, "A simple transmit diversity technique for wireless communications," IEEE J. Select. Areas Commun., vol. 16, no. 8, pp. 1451-1458, Oct. 1998.

[14] Ezio Biglieri, Coding for Wireless Channels, 1st ed., New York, NY: Springer, 2005.

[15] Arogyaswami Paulraj and Rohit Nabar and Dhananjay Gore, Introduction to SpaceTime Wireless Communications, 1st ed., New York, NY: Cambridge University Press, 2003.

[16] Andrea Goldsmith, Wireless Communications, 1st ed., New York, NY: Cambridge University Press, 2005.

[17] Theodore S. Rappaport, Wireless Communications: Principles and Practice, 2nd ed.,Prentice Hall, 2001.

[18] J.G. Proakis, Digital Communications, 3rd ed., New York, NY: McGraw-Hill, 1995.

[19] G. J. Foschini, "Layered space time architecture for wireless communication in a fading environment," Bell Labs Techinical Journal., vol. 1, no. 2, pp. 41-59, Autumn 1996.

[20] P. W. Wolniansky, G. J. Foschini, G. D. Golden, and R. A. Valenzuela, "V-blast: An architecture for realizing very high data rates over the rich-scattering wireless channel," Proc. Int. Symp. Signal, System, \& Electronics. (ISSE), pp. 295-300, Sep. 1998.

[21] Ran Gozali, Space-Time Codes for High Data Rate Wireless Communications, Ph.D. thesis, Virginia Polytechnic Institute and State University, Blacksburg, VA, April. 2002.

[22] Rose Trepkowski, "Channel estimation stratergies for coded mimo systems," M.S. thesis, Virginia Polytechnic Institute and State University, Blacksburg, VA, June. 2004.

[23] V. Tarokh, Ayman Naguib, N. Seshadri, and A.R. Calderbank, "Combining array processing and space-time coding," IEEE Trans. Commun., vol. 45, no. 4, pp. 11211128, May 1999.

[24] J. N. Laneman, Cooperative diversity in wireless networks: Algorithms and architectures, Ph.D. thesis, Massachusetts Institute of Technology, Cambridge, MA, August. 2002.

[25] Bin Zhao and M . C. Valenti, "Distributed turbo coded diversity for the relay channel," Electronics Letters, vol. 39, no. 10, pp. 786-787, May. 2003.

[26] G. D. Golden, C. J. Foschini, R. A. Valenzuela, and P. W. Wolniansky, "Detection algorithms and initial laboratory results using vblast space time architecture," Electronics Letters, vol. 35, no. 1, pp. 14-15, Jan. 1999. 